\title{
Electroweak and Higgs boson internal bremsstrahlung. General considerations for Majorana dark matter annihilation and application to MSSM neutralinos
}

\author{
Torsten Bringmann, ${ }^{a}$ Francesca Calore, ${ }^{b, d}$ Ahmad Galea ${ }^{a}$ and Mathias Garny ${ }^{c}$ \\ ${ }^{a}$ Department of Physics, University of Oslo, Box 1048, NO-0371 Oslo, Norway \\ ${ }^{b}$ LAPTh, CNRS, \\ 9 Chemin de Bellevue, BP-110, Annecy-le-Vieux, 74941, Annecy Cedex, France \\ ${ }^{c}$ Technical University Munich, James-Franck-Str. 1, D-85748 Garching, Germany \\ ${ }^{d}$ Université Savoie Mont Blanc, 27 Rue Marcoz, 73000, Chambéry, France \\ E-mail: torsten.bringmann@fys.uio.no, francesca.calore@lapth.cnrs.fr, \\ ahmad.galea@fys.uio.no, mathias.garny@tum.de
}

ABSTRACT: It is well known that the annihilation of Majorana dark matter into fermions is helicity suppressed. Here, we point out that the underlying mechanism is a subtle combination of two distinct effects, and we present a comprehensive analysis of how the suppression can be partially or fully lifted by the internal bremsstrahlung of an additional boson in the final state. As a concrete illustration, we compute analytically the full amplitudes and annihilation rates of supersymmetric neutralinos to final states that contain any combination of two standard model fermions, plus one electroweak gauge boson or one of the five physical Higgs bosons that appear in the minimal supersymmetric standard model. We classify the various ways in which these three-body rates can be large compared to the two-body rates, identifying cases that have not been pointed out before. In our analysis, we put special emphasis on how to avoid the double counting of identical kinematic situations that appear for two-body and three-body final states, in particular on how to correctly treat differential rates and the spectrum of the resulting stable particles that is relevant for indirect dark matter searches. We find that both the total annihilation rates and the yields can be significantly enhanced when taking into account the corrections computed here, in particular for models with somewhat small annihilation rates at tree-level which otherwise would not be testable with indirect dark matter searches. Even more importantly, however, we find that the resulting annihilation spectra of positrons, neutrinos, gamma-rays and antiprotons differ in general substantially from the model-independent spectra that are commonly adopted, for these final states, when constraining particle dark matter with indirect detection experiments.

KEywords: Beyond Standard Model, Cosmology of Theories beyond the SM, Supersymmetric Standard Model

ArXiv EPRINT: 1705.03466 


\section{Contents}

1 Introduction $\quad 2$

2 Majorana dark matter and relevant symmetries 4

2.1 Chiral symmetry, gauge symmetry and helicity suppression 4

$\begin{array}{ll}2.2 \text { Lifting of Yukawa and isospin suppression } & 6\end{array}$

2.3 Gauge invariance in IB processes 8

3 Neutralino annihilation to $\bar{f} f$ and an additional final-state particle $\quad 8$

3.1 Full analytic amplitudes and gauge-invariant subsets 9

$\begin{array}{ll}3.2 & \text { Helicity suppression in the MSSM } \\ \end{array}$

$\begin{array}{lll}3.2 .1 & \text { Yukawa suppression } & 11\end{array}$

$\begin{array}{lll}3.2 .2 & \text { Isospin suppression } & 12\end{array}$

$\begin{array}{lll}3.3 & \text { Yukawa and isospin suppression lifting } & 12\end{array}$

$\begin{array}{lll}3.4 & \text { Heavy propagator suppression } & 15\end{array}$

$\begin{array}{ll}3.5 & \text { Threshold effects } \\ \end{array}$

4 Double counting issues $\quad 19$

$\begin{array}{ll}\text { 4.1 Narrow width approximation and total cross section } & 19\end{array}$

$\begin{array}{lll}4.2 & \text { Spectrum of stable particles } & 21\end{array}$

5 Results for the MSSM $\quad 24$

5.1 Theoretical benchmark models 25

$\begin{array}{lll}5.2 & \text { Total annihilation rate } & 26\end{array}$

$\begin{array}{lll}5.3 & \text { Yield enhancement } & 29\end{array}$

$\begin{array}{lll}5.4 & \text { Indirect detection spectra } & 31\end{array}$

$\begin{array}{lll}6 & \text { Conclusions } & 38\end{array}$

A Neutralino annihilation amplitudes $\quad 39$

$\begin{array}{ll}\text { A.1 Expansion of amplitudes in the helicity basis } & 39\end{array}$

A.2 Results for expanded amplitudes 42

A.3 Suppression lifting from individual diagrams 49

B Numerical implementation $\quad 52$

$\begin{array}{ll}\text { C Spin correlations of decaying resonances } & 54\end{array}$ 


\section{Introduction}

The prime hypothesis for the cosmologically observed dark matter (DM) [1] is a new type of elementary particle [2]. Among theoretically well-motivated candidates, weakly interacting massive particles (WIMPs) play a prominent role. This is because such WIMPs very often appear in theories that attempt to cure the fine-tuning problems in the Higgs sector of the standard model of particle physics (SM), and because thermal relics with weak masses and cross sections at the electroweak scale are typically produced with the correct abundance to account for the DM density today [3,4]. Another advantage is that the WIMP hypothesis can be tested in multiple ways: at colliders, where the signature consists in missing energy, in direct detection experiments aiming to observe DM particles recoiling off the nuclei of deep underground detectors, and in indirect searches for the debris of DM annihilation in cosmic regions with large DM densities. Direct detection experiments have become extremely competitive in constraining smaller and smaller scattering rates [5, 6], and collider searches have pushed the scale of new physics to $\mathrm{TeV}$ energies in many popular models $[7,8]$. It is worth stressing, however, that only 'indirect' searches would eventually allow to test the WIMP DM hypothesis in situ, i.e. in places that are relevant for the cosmological evidence for DM. Also indirect searches have become highly competitive during the last decade, now probing the 'thermal cross section' (the one that is needed to produce the observed DM abundance) up to WIMP masses of the order of $100 \mathrm{GeV}[9,10]$.

A key quantity for both thermal production of WIMPs and indirect searches is the total annihilation cross section. Multiplied by the relative velocity $v$ of the incoming DM particles, it can in the non- relativistic limit be expanded as

$$
\sigma v=a+b v^{2}+\mathcal{O}\left(v^{4}\right)
$$

It was noted early $[11,12]$ that radiative corrections to $\sigma v$ can be huge because of symmetries of the annihilating DM pair in the $v \rightarrow 0$ limit. For indirect DM searches, changes in either the partial cross section, for a given annihilation channel, or the differential cross section, $d \sigma v / d E$, may be phenomenologically even more important. The reason is that an additional photon in the final state can give rise to pronounced spectral features in the DM signal in both gamma [13] and charged cosmic rays [14]. For electroweak corrections, the situation is in some sense even more interesting because, on top of the just mentioned effects, completely new indirect detection channels may open up. In this way, antiproton data can for example efficiently constrain DM annihilation to light leptons when considering the associated emission of $W$ or $Z$ bosons [15]. In the presence of point-like interactions, such as described by effective operators, the resulting spectra can be computed in a model-independent way by using splitting functions inspired by a parton picture [16]. This approach is very useful for generic DM phenomenology and is, for example, the one implemented in the 'cookbook' for indirect detection [17]. One of the main results of this article (see also [18]) is that the resulting cosmic ray spectra from DM annihilation can differ substantially from the actual spectra, calculated in a fully consistent way from the underlying particle framework. 
Here we revisit in detail one of the most often discussed examples where radiative corrections can be large, namely the case of a Majorana DM particle $\chi$. The tree-level annihilation rate into light fermions $f$ is then on general grounds 'helicity suppressed', for $v \rightarrow 0$, as a consequence of the conserved quantum numbers of the initial state [19]. The resulting suppression by a factor of $m_{f}^{2} / m_{\chi}^{2}$ can be lifted by allowing for an additional vector [11] or scalar [20] boson in the final state, implying that for DM masses at the electroweak scale the radiative 'corrections' can be several orders of magnitude larger than the result from lowest order in perturbation theory. ${ }^{1}$ Here, we revisit these arguments and point out that the effect commonly referred to as helicity suppression is in fact the culmination of two distinct suppression mechanisms, in the sense that they can be lifted independently. This results, in general, in a rather rich phenomenology of such radiative corrections.

As an application, we consider electroweak corrections to the annihilation cross section of the lightest supersymmetric neutralino - one of the most often discussed DM prototypes [28] and still a leading candidate despite null searches for supersymmetry at ever higher energies and luminosities at the LHC $[7,8]$ - though our main findings can be extended in an analogous way to other DM candidates that couple to the SM via the electroweak or Higgs sector. Concretely, we provide a comprehensive analysis, both analytically and numerically, of all three-body final states from neutralino annihilation that contain a fermion pair and either an electroweak gauge boson or one of the five Higgs bosons contained in the minimal supersymmetric standard model (MSSM), for a neutralino that can be an arbitrary admixture of Wino, Bino and Higgsino. ${ }^{2}$ We find large parameter regions where these three-body final states significantly enhance the DM annihilation rate, with the impact on the shape of the cosmic-ray spectra relevant for indirect detection being even more significant.

One of the technically most involved aspects, apart from the shear number of diagrams to be considered, is how to avoid 'double counting' the on-shell parts of the three-body amplitudes that are already, implicitly, included in the corresponding two-body results. We provide an in-depth treatment of this issue and demonstrate how to accurately treat not only the total cross section but also the resulting cosmic-ray spectra. We again find significant effects on the latter, indicating the need to correctly adopt this method also for other DM candidates. In fact, in order to reliably test the underlying particle models, our findings suggest that at least for fermionic final states it is in general not sufficient to use the model-independent spectra traditionally provided by numerical packages. The numerical routines that implement our results for the neutralino case will be fully available with the next public release of DarkSUSY [39, 40].

\footnotetext{
${ }^{1}$ The lifting of helicity suppression via three-body final states is also relevant for real scalar dark matter [21-25] and, under certain conditions, for vector dark matter [26]. The case in which the additional boson is a $Z^{\prime}$ has been considered in [27].

${ }^{2}$ For neutralino annihilation, so far only the cases of photon [13] and gluon [29] internal bremsstrahlung (IB) have been considered in full generality. Final states with electroweak gauge bosons have been considered for pure binos in [30-35], for Higgsinos in [36], and for pure Winos in [37]. A first study for a general neutralino has been performed in [18]. Finally, final states involving the SM-like Higgs boson have been considered in [20] for a toy model encompassing a pure Bino (see also [38]).
} 
This article is organized as follows. We start in section 2 with a general discussion of Majorana DM annihilating to fermions and the relevant symmetries that arise for $v \rightarrow 0$, revisiting in particular the often invoked 'helicity suppression' arguments and how this suppression can be lifted fully or partially. In section 3 we then consider the concrete case of neutralino DM, and discuss the various possibilities of how the presence of an additional final state boson can add sizeable contributions to, or even significantly enhance, the two-body annihilation rates. The double counting issues mentioned above are then addressed in detail in a separate section 4 . We scan the parameter space of several MSSM versions and demonstrate the effect of these newly implemented corrections to neutralino annihilation in section 5 , both for the annihilation rates and the cosmic-ray spectra relevant for indirect DM searches, and present our conclusions in section 6. In a more technical appendix, we describe the details of our analytical calculations to obtain the three-body matrix elements for fully general neutralino annihilation in the MSSM (appendix A), the numerical implementation of these results in DarkSUSY (appendix B), and how to correctly treat spin correlations of decaying resonances (appendix $\mathrm{C}$ ).

\section{Majorana dark matter and relevant symmetries}

For DM annihilation in the Milky Way halo, where DM particles have typical velocities of order $10^{-3}$, only the first term in eq. (1.1) gives a sizeable contribution. In the following we therefore neglect $p$ and higher partial wave contributions, and it is understood that all (differential) cross sections are effectively evaluated in the zero-velocity limit. For an $s$-wave, the relative angular momentum in the initial state is $L=0$. Due to the Majorana nature the initial particles are identical, but because we consider fermions the total wave function still needs to be antisymmetric with respect to exchanging the incoming particles. The orbital wave function for $L=0$ is symmetric, so in order to get an anti-symmetric total wave function the spins must couple into an antisymmetric state. This is only possible for the singlet state, with $S=0$, resulting in the following quantum numbers:

$$
J=0, \quad C=(-1)^{L+S}=1, \quad P=(-1)^{L+1}=-1 .
$$

Here, the general expressions for $C$ and $P$ apply because we have a system of two fermions. Assuming no significant sources of $C P$ violation in the theory, which generally are highly constrained by measurements of the electric dipole moment and other precision experiments, the symmetry of the final state is hence also restricted to be $J_{C P}=0_{-}$. This implies the well-known 'helicity' suppression of the annihilation rate into light fermions, similar to the case of charged pion decay. In the following we first briefly review the origin of this suppression, and then argue that it can in fact be related to a combination of two rather independent suppression mechanisms.

\subsection{Chiral symmetry, gauge symmetry and helicity suppression}

We want to study Dirac fermions $f$ as possible final states from the annihilation of Majorana particles $\chi$. Their free Lagrangian is given by $\mathcal{L}_{0}=\bar{f}\left(i \not \partial-m_{f}\right) f$, which is invariant under Lorentz transformations, i.e. invariant under $\mathrm{SU}(2)_{L+R}$. In the massless limit, $m_{f} \rightarrow 0$, 
this symmetry is upgraded to a chiral symmetry $\mathrm{SU}(2)_{L} \times \mathrm{SU}(2)_{R}$, in which the left and right handed Weyl states transform independently of each other, and helicity and chiral eigenstates unify. For a fermion pair $\bar{f} f$, the spins can combine to either a singlet $(S=0)$,

$$
(|\uparrow \downarrow\rangle-|\downarrow \uparrow\rangle) / \sqrt{2}
$$

or a triplet $(S=1)$ spin state,

$$
\{|\downarrow \downarrow\rangle, \quad(|\uparrow \downarrow\rangle+|\downarrow \uparrow\rangle) / \sqrt{2}, \quad|\uparrow \uparrow\rangle\},
$$

where the arrows indicate the spin direction along the $z$-axis (the first entry refers to the antifermion, the second to the fermion). If the two fermion momenta are (anti-)parallel e.g. because they are emitted back-to-back as the final states of a DM annihilation process - the $z$-axis can be chosen to be aligned in the same direction as the momenta, and the above spin projections on the $z$ axis are directly related to the helicities of the two particles. Choosing $\mathbf{p}_{f}\left(\mathbf{p}_{\bar{f}}\right)$ to point along the positive (negative) $z$-axis, the helicity configurations $h=S_{z} p_{z} /\left|p_{z}\right|$ of the singlet and triplet state are then given by

$$
\frac{1}{\sqrt{2}}\left(\left|-\frac{1}{2},-\frac{1}{2}\right\rangle-\left|+\frac{1}{2},+\frac{1}{2}\right\rangle\right) \rightarrow \frac{1}{\sqrt{2}}\left(\left|\bar{f}_{R}, f_{L}\right\rangle-\left|\bar{f}_{L}, f_{R}\right\rangle\right)
$$

and

$$
\begin{aligned}
& \left\{\left|+\frac{1}{2},-\frac{1}{2}\right\rangle, \frac{1}{\sqrt{2}}\left(\left|-\frac{1}{2},-\frac{1}{2}\right\rangle+\left|+\frac{1}{2},+\frac{1}{2}\right\rangle\right),\left|-\frac{1}{2},+\frac{1}{2}\right\rangle\right\} \\
& \rightarrow\left\{\left|\bar{f}_{L}, f_{L}\right\rangle, \frac{1}{\sqrt{2}}\left(\left|\bar{f}_{R}, f_{L}\right\rangle+\left|\bar{f}_{L}, f_{R}\right\rangle\right),\left|\bar{f}_{R}, f_{R}\right\rangle\right\},
\end{aligned}
$$

where the arrows indicate the chiral states in the left/right decoupling limit, i.e. for $m_{f} \rightarrow 0$.

The momentum configuration thus restricts which helicity states can be associated to the spin states. Angular momentum and the assumed $C P$ invariance, on the other hand, restrict which spin state can be realized. Since $C P=(-1)^{L+S} \times(-1)^{L+1}=(-1)^{S+1}$, for example, only the singlet state with $S=0$ is compatible with the odd $C P$ parity of the initial state. Eq. (2.4) then tells us that both fermion and antifermion in this momentum configuration must have the same helicity. In any chirally symmetric theory, however, the antifermion must necessarily have the opposite helicity of the fermion. We note that angular momentum conservation alone leads to the same conclusion: since $\mathbf{L}=\mathbf{r} \times \mathbf{p}$, we must have $L_{z}=0$ and hence $S_{z}=J_{z}=0$. Eqs. (2.4), (2.5) then imply that fermion and antifermion must, independently of the value of $S$, have the same helicity. The annihillation process $\chi \chi \rightarrow \bar{f} f$ is therefore only possible if chiral symmetry is broken in the Lagrangian, for example through an explicit fermionic mass term $m_{f} \bar{f}_{L} f_{R}$ or through the coupling of the fermion $f$ to a scalar field $\lambda \phi \bar{f}_{L} f_{R}$. It follows that the amplitude of the annihilation process must be proportional to the chiral symmetry breaking parameters $m_{f}$ or $\lambda$.

In addition, it is instructive to consider also the isospin of the involved particles. Since left-and right-handed SM fermions transform under different representations of SU $(2)_{L}$, the final states $\bar{f}_{L} f_{R}$ and $\bar{f}_{R} f_{L}$ have total isospin $I=1 / 2$. The initial state $\chi \chi$, on the other 
hand, has necessarily integer isospin, implying $\Delta I \neq 0$. The annihilation rate thus has to vanish for an unbroken $\mathrm{SU}(2)_{L}$, and therefore has to be proportional to at least one power of the Higgs vacuum expectation value (VEV) $v_{E W}$. For heavy DM the ratio $\delta_{v} \equiv v_{E W} / m_{\chi}$ becomes small, and processes with $\Delta I \neq 0$ will be suppressed by some power of $\delta_{v}$.

In total, this implies that the amplitude for $\chi \chi \rightarrow \bar{f} f$ has to involve (at least) one parameter that breaks chiral symmetry, and one power of $v_{E W}$ that controls breaking of the $\mathrm{SU}(2)_{L}$ symmetry. For the SM fermions that receive their mass from the Higgs mechanism, both of these conditions are fulfilled for the usual helicity suppression factor $m_{f}$. Depending on the model, however, there can be further possibilities, as we will discuss in detail for the case of the MSSM below, and it is useful to discriminate between the two suppression mechanisms. In the following, we therefore refer to the suppression related to chiral symmetry breaking as Yukawa suppression, and to the one related to electroweak symmetry breaking as isospin suppression. While isospin suppression is controlled by only one parameter, $\delta_{v}=v_{E W} / m_{\chi}$, there can in principle be several sources of chiral symmetry breaking, for example in models with more complicated Higgs sectors. Nevertheless, as we discuss in detail in section 3, all terms that break chiral invariance in the MSSM are accompanied by Yukawa couplings $y_{f} \propto m_{f} / v_{E W}$. Even though the following discussion of suppression lifting is completely model independent we will thus continue to assume, for concreteness, that chiral symmetry breaking is controlled by $y_{f}$.

\subsection{Lifting of Yukawa and isospin suppression}

With the above discussion in mind, the only way to avoid the suppression of non-relativistic Majorana DM annihilation is to allow for an additional final state particle. Lorentz invariance requires this additional particle to be a boson, such that the leading process we are interested in is of the form

$$
\chi \chi \rightarrow B \bar{F} f
$$

where $B$ is a scalar or vector boson, and $F=f$ if $B$ is electrically neutral. The additional boson can be either a SM particle, in particular a photon (refered to as electromagnetic IB), a gluon, a weak gauge boson $\left(W^{ \pm}, Z\right)$ or the Higgs boson $h$, or it can be a new particle beyond the SM (for example a heavy Higgs boson within the MSSM). For the moment we want to keep the discussion model-independent, and therefore focus on the former case. A frequently used approximation is to restrict the discussion to $B$ being radiated off a fermion line in the final state, as described by soft and/or collinear splitting functions [16, 17]. We emphasize that this approach does not capture the (partial) lifting of helicity suppression, and therefore is inadequate for the case of heavy Majorana DM annihilation to fermions.

Taking the gauge restoration limit $v_{E W} \rightarrow 0$, it becomes straight-forward to exhibit the scaling of a given process with $y_{f}$ and $v_{E W}$. (We emphasize that we consider this limit only in order to discuss the possible mechanisms of Yukawa and isospin suppression lifting, while all our numerical results later on take the full dependence on $v_{E W}$ and $y_{f}$ into account). In this limit, the left- and right-handed components of the fermions in the final state and in internal lines can not only be considered as gauge interaction eigenstates but as independently propagating degrees of freedom. The fermion mass is treated perturbatively in the mass in- 


\begin{tabular}{|c|c|c|c|c|c|c|}
\hline & $g$ & $Z_{T} / \gamma$ & $W_{T}$ & $Z_{L}$ & $W_{L}$ & $h$ \\
\hline $\bar{F}_{R} f_{L}$ or $\bar{F}_{L} f_{R}$ & $y_{f} v_{E W}$ & $y_{f} v_{E W}$ & $y_{f} v_{E W}$ & $y_{f}$ & $y_{f}$ & $y_{f}$ \\
$\bar{F}_{L} f_{L}$ & 1 & 1 & 1 & $v_{E W}$ & $v_{E W}$ & $v_{E W}$ \\
$\bar{F}_{R} f_{R}$ & 1 & 1 & 1 & $v_{E W}$ & $v_{E W}$ & $v_{E W}$ \\
\hline
\end{tabular}

Table 1. Summary of Yukawa and isospin suppression(-lifting) in three-body annihilation processes $\chi \chi \rightarrow B \bar{F} f$ for various final state boson $B$ and fermion combinations. Entries $\propto 1$ correspond to processes that potentially can lift both Yukawa and isospin suppression of the two-body process. Entries $\propto y_{f}$ can lift isospin suppression but are still suppressed by the Yukawa coupling, while those $\propto v_{E W}$ can lift Yukawa suppression but are still suppressed by $\delta_{v}=v_{E W} / m_{\chi}$ for large $m_{\chi}$.

sertion approximation, and is associated with a chirality flip along with a suppression factor $m_{f} \propto y_{f} v_{E W}$. In addition, longitudinally polarized gauge bosons $W_{L} / Z_{L}$ can be replaced by the corresponding Goldstone bosons $G^{ \pm}, G^{0}$ by virtue of the Goldstone boson equivalence theorem, cf. eqs. (2.7)-(2.8) below. All final states thus have definite $\mathrm{SU}(2)_{L}$ quantum numbers (i.e. $I=1 / 2$ for $G^{ \pm}, G^{0}, h, f_{L}, I=0$ for $f_{R}$, and $I=1$ for $W_{T}$ ), except for the $Z_{T}$, which is a mixture of $I=0,1$ states (even in the gauge restauration limit, we find it convenient to express our results in terms of the $Z$ boson instead of the neutral $\mathrm{SU}(2)_{L}$ boson).

The amplitude of the generic three-body process indicated in eq. (2.6) can be non-zero for $v_{E W} \rightarrow 0$ only if $\Delta I=0$, i.e. if isospin is conserved. Furthermore, the amplitude must vanish for $y_{f} \rightarrow 0$ unless both fermions have the same chirality. Note that this is possible for three-body processes because the kinematics does not force the fermions to be emitted back-to-back in the center-of-mass (CMS) frame, and therefore the arguments discussed in section 2.1 do not apply. ${ }^{3}$ These two observations immediately determine which annihilation processes can lift either Yukawa or isospin suppression (or both). In table 1, we show schematically the required scaling of the amplitude that results from these considerations, for various combinations of fermion chiralities and final state bosons (where the longitudinal gauge bosons represent the corresponding Goldstone bosons). Both suppression factors can be lifted only in processes where a transverse gauge boson $\left(Z_{T}\right.$, $W_{T}, \gamma$, or gluon $g$ ) is emitted and the final state fermions are described by spinors of equal chirality $\left(\bar{F}_{R} f_{R}\right.$ or $\left.\bar{F}_{L} f_{L}\right)$. For longitudinal gauge bosons $\left(Z_{L}\right.$ or $\left.W_{L}\right)$ or the Higgs boson $h$, only one of the suppression factors can (potentially) be avoided for three-body final states: isospin suppression can be lifted if the fermions are of opposite chirality, and Yukawa suppression can be lifted if the fermions are of equal chirality.

\footnotetext{
${ }^{3}$ In the extreme case where both fermions are emitted in the same direction, e.g., one simply has to exchange $f_{R} \leftrightarrow f_{L}$ in eqs. (2.4), (2.5), which allows equal chiralities of the fermions in both the singlet and triplet spin state. In this kinematical configuration, it is easy to visualize how the fermion momentum can be balanced by the emitted boson $B$, and how their spin can combine with $S_{B}$ and $L$ to the required $J=0$ for both $S_{B}=0$ and $S_{B}=1$. In general, the spin singlet and triplet states will be linear combinations of all chiral states, with expectation values that depend on the angle between the fermion momenta, thus rendering the above argument essentially independent of the specific kinematical configuration. Also the requirement of $C P$ conservation is much less restrictive for three-body than for two-body final states. A general discussion is somewhat complicated by the fact that e.g. $\bar{F} f$ is not necessarily a $C P$ eigenstate that could be analysed individually, but in principle straight-forward by classifying all possible effective operators that connect initial and final states (similar in spirit to the analysis of two-body final states presented in ref. [41]).
} 
Let us stress that the symmetry arguments presented above simply guarantee that the amplitude must vanish for $y_{f} \rightarrow 0$ and $v_{E W} \rightarrow 0$, respectively, and the same applies to any gauge invariant sub-sets of diagrams. The actual suppression can thus be stronger than indicated by table 1 , i.e. by additional powers of $v_{E W}$ or $y_{f}$. At the same time, we caution that single diagrams can scale in a different way, depending on the gauge choice, such that the vanishing for $y_{f} \rightarrow 0$ or $v_{E W} \rightarrow 0$ is in general not guaranteed.

\subsection{Gauge invariance in IB processes}

Following up on the last comment, let us for convenience briefly recall how to verify gauge independence and identify gauge invariant subsets of diagrams. While for photon emission a good test is to check whether a given set of diagrams satisfies the Ward identity $\mathcal{M}^{\mu}(\chi \chi \rightarrow$ $\bar{f} f \gamma) k_{\mu}=0$, where $k_{\mu}$ is the momentum of the photon, this does not work for electroweak IB because $\mathrm{SU}(2)_{L} \times \mathrm{U}(1)_{Y}$ has been spontaneously broken. Indeed the question of gauge invariance changes in general, as weak hypercharge and isospin are no longer conserved in their original form. For the spontaneously broken Glashow-Weinberg-Salam theory the correct way to define gauge invariance is in terms of the preserved BRST symmetry [42, 43], under which SM field transformations involve ghost fields which arise from the electroweak gauge fixing procedure. This implies a new set of Ward identities, which in general depend on the choice of gauge. Using the standard $R_{\xi}$ class of gauges [44], we arrive at the Ward identities for electroweak IB as expected from the Goldstone equivalence theorem:

$$
\begin{aligned}
\mathcal{M}^{\mu}(\chi \chi \rightarrow \bar{f} f Z) k_{\mu} & =i m_{Z} \mathcal{M}\left(\chi \chi \rightarrow \bar{f} f G^{0}\right), \\
\mathcal{M}^{\mu}\left(\chi \chi \rightarrow \bar{F} f W^{ \pm}\right) k_{\mu} & =m_{W} \mathcal{M}\left(\chi \chi \rightarrow \bar{F} f G^{ \pm}\right) .
\end{aligned}
$$

We reiterate that eqs. (2.7) and (2.8) in general apply to (subsets of) the full amplitude, not individual diagrams, and are a valuable test for the results outlined in the next section.

\section{Neutralino annihilation to $\bar{f} f$ and an additional final-state particle}

In this section we apply the general discussion of helicity suppression lifting in Majorana DM annihilation to the lightest supersymmetric neutralino as DM candidate, and additional final state bosons charged under $\mathrm{SU}(2)_{L}$. For photon or gluon IB we refer to the references listed in the introduction. Concerning the choice of DM candidate, we note that much of the following discussion is still rather generic and can thus be extended in a straight-forward way to any theory with an extended Higgs sector or where the DM particles belong to a different electroweak multiplet. We will introduce the relevant three-body processes and Feynman diagrams in section 3.1, re-visit the discussion of the helicity suppression in light of the specific situation encountered in the MSSM (section 3.2) and then demonstrate in detail how these suppressions can be lifted, fully or partially, in section 3.3. In sections 3.4 and 3.5, finally, we discuss two mechanisms by which three-body cross sections can be enhanced which are not related to the helicity suppression of two-body final states. 

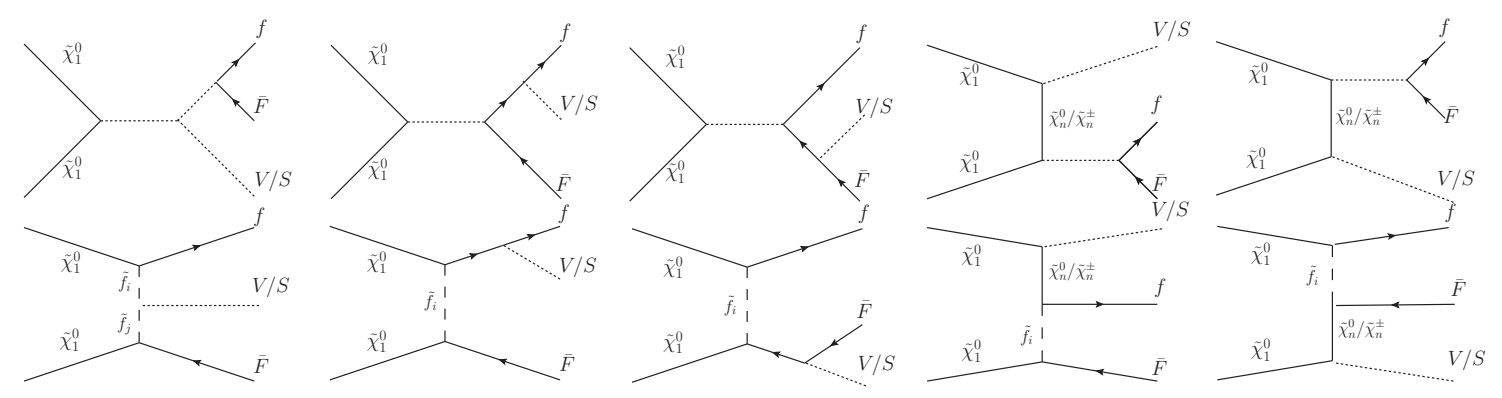

Figure 1. Condensed representation of all Feynman diagrams for neutralino annihilation into $\bar{F} f V$ or $\bar{F} f S$, where dotted lines indicate scalar $\left(S=A, h, H, H^{ \pm}\right)$or vector $\left(V=Z, W^{ \pm}\right)$mediators, depending on the final state configuration. Fermion final states are identical, $F=f$, for neutral boson emission $\left(h, H, A\right.$ or $Z$ ), while $(f, F)$ constitute the two components of an $\mathrm{SU}(2)_{L}$ doublet for charged boson emission $\left(H^{ \pm}\right.$or $\left.W^{ \pm}\right)$. See text for more details on how the individual topologies are referred to in this article.

\subsection{Full analytic amplitudes and gauge-invariant subsets}

From now on, we thus assume DM to be composed of the lightest neutralino, $\chi \equiv \tilde{\chi}_{1}^{0}$, which is a superposition of Wino, Bino and Higgsino states,

$$
\chi=N_{11} \tilde{B}+N_{12} \tilde{W}^{3}+N_{13} \tilde{H}_{1}^{0}+N_{14} \tilde{H}_{2}^{0},
$$

obtained by diagonalizing the neutralino mass matrix

$$
\left(\begin{array}{cccc}
M_{1} & 0 & \frac{-g^{\prime} v_{1}}{\sqrt{2}} & \frac{g^{\prime} v_{2}}{\sqrt{2}} \\
0 & M_{2} & \frac{g v_{1}}{\sqrt{2}} & \frac{-g v_{2}}{\sqrt{2}} \\
\frac{-g^{\prime} v_{1}}{\sqrt{2}} & \frac{g v_{1}}{\sqrt{2}} & 0 & -\mu \\
\frac{g^{\prime} v_{2}}{\sqrt{2}} & \frac{-g v_{2}}{\sqrt{2}} & -\mu & 0
\end{array}\right) .
$$

Here, $M_{1}$ and $M_{2}$ are the Bino and Wino mass parameters, respectively, and $\mu$ is the Higgsino mass parameter; $v_{1}$ and $v_{2}$ are the VEVs of the two Higgs doublets, with $v_{E W}=$ $\sqrt{v_{1}^{2}+v_{2}^{2}}$ and $\tan \beta \equiv v_{1} / v_{2}$, and $g$ and $g^{\prime}$ are the $\mathrm{SU}(2)_{L}$ and $\mathrm{U}(1)_{Y}$ couplings, respectively. We follow the conventions of ref. [45], as implemented in DarkSUSY, and take all mass eigenvalues to be positive, while the diagonalization matrix $N$ can be complex.

We want to consider here all three-body final states that contains a fermion pair and a boson that is charged under $\mathrm{SU}(2)_{L}$. Assuming $C P$-violating terms to be small, the full list of processes of interest is thus

$$
\chi \chi \rightarrow W^{+} \bar{F} f, Z \bar{f} f, H^{+} \bar{F} f, A \bar{f} f, H \bar{f} f, h \bar{f} f .
$$

Here, $A$ denotes the $C P$-odd Higgs, $H^{+}$the charged Higgs, and $H$ and $h$ the heavy and light $C P$-even Higgs bosons, respectively. For charged boson final states, $f$ denotes any fermion doublet component with isospin $+1 / 2$, and $F$ the corresponding one with isospin $-1 / 2$; for neutral bosons, $f$ can be any SM fermion. 


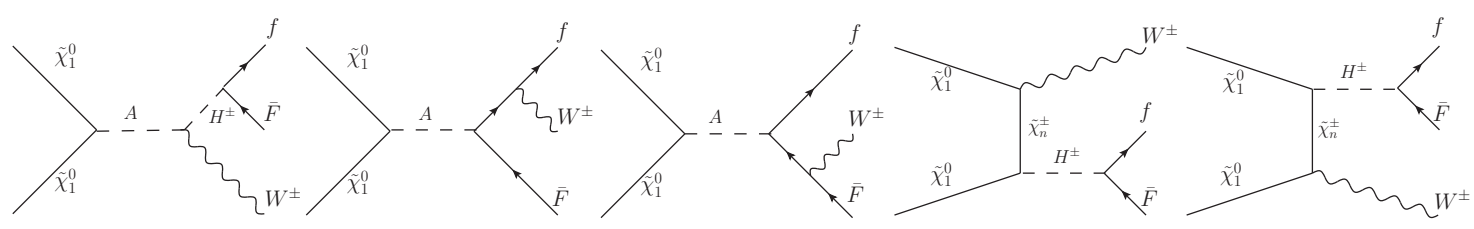

Figure 2. Gauge invariant set of amplitudes for neutralino DM annihilation into a fermion pair and a $W$ boson, mediated by $s$-channel bosons with a mass at the scale of the $C P$-odd Higgs $A$.

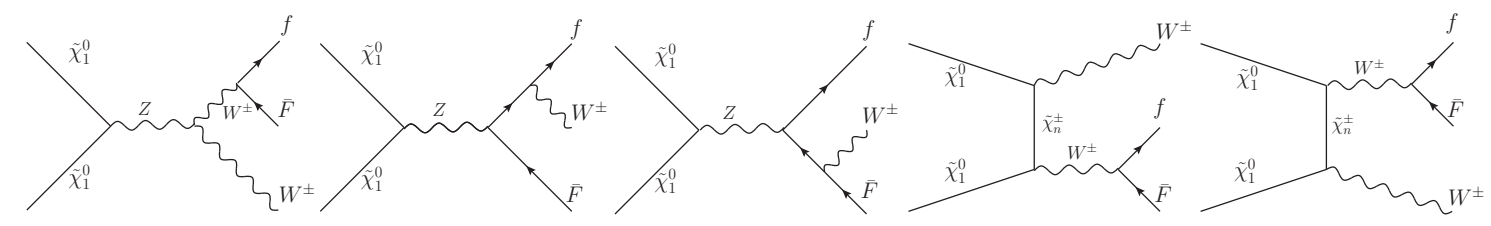

Figure 3. Same as figure 2, but mediated by $s$-channel bosons with a mass at the electroweak scale.

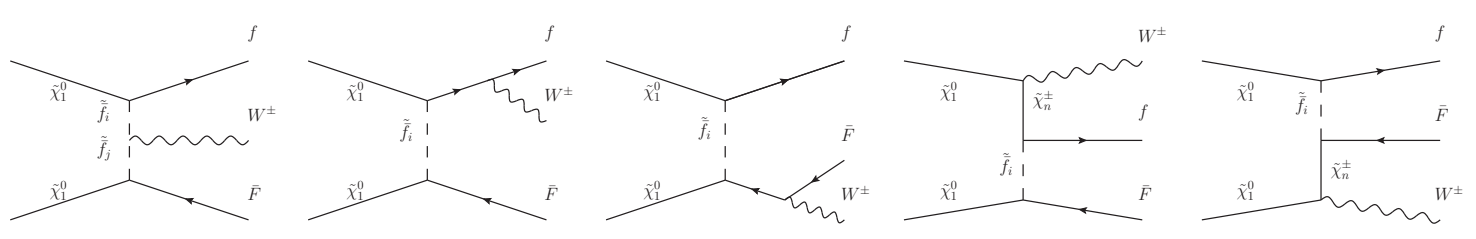

Figure 4. Same as figure 2, but mediated by $t$-channel sfermions.

In figure 1, we show all contributing Feynman diagrams in a condensed form (note that some of these diagrams may vanish for specific combinations of internal and external particles). For future reference, we follow ref. [18] and refer to the top row of diagrams as (derived from two-body) s-channel processes, and to the bottom row of diagrams as $t / u$-channel processes (noting that $t$ - and $u$-channel amplitudes are identical in the $v \rightarrow 0$ limit). Likewise, we denote diagrams of the type that appear in the first column as virtual internal bremsstrahlung (VIB), diagrams of the type that appear in the second and third column as final state radiation (FSR), ${ }^{4}$ and diagrams of the type that appear in the last two columns as initial state radiation (ISR).

We explicitly calculate the full analytical expressions for all these processes in the limit of vanishing relative velocity of the annihilating neutralino pair, see appendix A.1 for technical details. We then use the Ward identities in eqs. (2.7) and (2.8) to group diagrams into gauge invariant sets for the case of vector boson final states. In general we identified only two of such invariant sets: those diagrams that are derived from two-body $s$-channel processes and those that are derived from two-body $t$-channel processes. In the limit $m_{A} \gg m_{\chi}$ - which is phenomenologically particularly relevant because the observed

\footnotetext{
${ }^{4}$ We stress that this distinction between VIB and FSR, while useful for the specific purpose of our discussion, is not gauge invariant and exclusively refers to the topology of the involved diagrams. In particular, it should not be confused with an often used gauge invariant alternative set of definitions where FSR refers exclusively to the soft or collinear photons radiated from the final legs [13, 16, 17], while VIB is defined as the difference between the full amplitude squared and the FSR contribution [13].
} 
Higgs is very SM like - the $s$-channel diagrams however split into two gauge-invariant subsets. All diagrams then fall quite neatly into 3 categories: heavy Higgs s-channel, which are the set of diagrams with (at least one) mediator at the mass scale $M_{A}$ (see figure 2), weak-scale s-channel, which are the set of diagrams with $s$-channel mediators at the weak scale (see figure 3 ), and $t$-channel, which are the set of diagrams with sfermion mediators (see figure 4). ${ }^{5}$ For $Z \bar{f} f$ and $h \bar{f} f$ final states the three sets of diagrams can be obtained analogously: $t$-channel contributions involve at least one sfermion line, while the remaining diagrams belong to the $s$-channel category (which can be further split into subsets involving at least one mediator at scale $M_{A}$, or none, respectively).

\subsection{Helicity suppression in the MSSM}

As established in the previous section, the 'helicity suppression' of the two-body annihilation rate by a factor of $m_{f}^{2} / m_{\chi}^{2}$ is indeed the combination of in principle independent Yukawa and isospin suppressions. Let us now turn back to this observation and discuss it in more detail in light of the MSSM, where both mechanisms are still intrinsically linked because of the connection between gauge symmetry and chiral structure in the MSSM Lagrangian.

\subsubsection{Yukawa suppression}

The chiral symmetry of the MSSM Lagrangian is broken by terms proportional to Yukawa couplings (in order to avoid flavour-changing neutral currents, we assume as usual that the $A$-terms are proportional to the Yukawa coupling matrices). Following the general arguments of section 2.1, any amplitude contributing to $\chi \chi \rightarrow \bar{f} f$ must therefore be proportional to $y_{f}$. Within the MSSM the values of $y_{f}$ are functions of $\tan \beta$ but, except for the top quark, in general so small that this can lead to a suppression of the two-body amplitudes by many orders of magnitude. From the point of view of the broken theory, this Yukawa suppression appears to arise from rather different types of contributions to the Lagrangian:

i) fermion mass terms

ii) couplings of any of the five physical Higgs fields to fermions

iii) couplings of fermions to sfermion mass eigenstates (which mix the left- and righthanded fields).

For example, the first case is relevant for annihilation into fermions via $t$-channel sfermion exchange if the sfermion mixing is small (otherwise, the third contribution can dominate the amplitude), and the second for annihilation via $s$-channel pseudoscalar mediation.

We note that all three interaction types couple left- and right-handed states and hence can 'flip' the helicity of one of the final state fermions. The helicity combinations that

\footnotetext{
${ }^{5}$ We note that for $v \rightarrow 0$ the two $s$-channel ISR diagrams are actually identical, but for clarity we still include them separately in figures 2 and 3. For $v \rightarrow 0$ and $m_{F} \rightarrow 0$, also the two $t$-channel ISR diagrams are identical; in practice, the difference only matters for final states containing top and bottom quarks. As an important cross-check of our final amplitudes, we confirmed analytically that these identities indeed hold.
} 
would result in a chirally symmetric theory, $\bar{f}_{R, L} f_{R, L}$, can thus be transformed into those compatible with the global symmetry requirements outlined in section $2.1, \bar{f}_{R, L} f_{L, R}$. Traditionally, the notion of this helicity flip is sometimes taken to refer specifically to the case (i), in which it is the (kinematic) fermion mass that breaks chiral symmetry in the Lagrangian. Instead, we associate the effect directly with the Yukawa couplings in the MSSM Lagrangian (which of course give rise to the SM fermion masses).

\subsubsection{Isospin suppression}

As also discussed in section 2.1, the annihilation process $\chi \chi \rightarrow \bar{f} f$ furthermore violates weak isospin, $\Delta I \neq 0$, and therefore its amplitude has to vanish in the gauge restoration limit $v_{E W} \rightarrow 0$. The resulting isospin suppression by a factor $\delta_{v} \equiv v_{E W} / m_{\chi}$, for heavy neutralinos, can arise from different terms in the Lagrangian of the broken theory:

a) fermion mass terms

b) mixing of different gauge multiplets (Bino, Higgsino, Wino) that contribute to the lightest neutralino mass eigenstate given by eq. (3.1)

c) mixing of left- and right-handed sfermion eigenstates.

The structure of the neutralino mass matrix (3.2) indeed confirms that neutralino mixings vanish for $v_{E W} \rightarrow 0$, as required by $\mathrm{SU}(2)_{L}$ invariance. Note that case (a) and (c) are intrinsically linked to an accompanying chirality violation, since $m_{f} \propto y_{f} v_{E W}$ and the offdiagonal terms in the sfermion mass matrix are also proportional to $y_{f}$ within the MSSM. Let us consider as an illustration the $t$ - and $s$-channel contributions to $\chi \chi \rightarrow \bar{f} f$. The kinematical helicity suppression due to the fermion mass $m_{f}$ is relevant for the $t$-channel (sfermion exchange). In this case Yukawa and isospin suppression simply arise from the two factors in $m_{f} \propto y_{f} v_{E W}$ (case (a) and (i), respectively). In addition, the Yukawa and isospin violation can be due to the sfermion mixing (case (c) and (iii)). Indeed, due to the mixing, a given sfermion mass eigenstate can couple to both left- and right-handed fermions, which then gives rise to the required chirality flip.

For $s$-channel annihilation, on the other hand, the situation is more interesting in the sense that Yukawa and isospin suppression cannot simply be traced back to the same origin. For a pseudoscalar Higgs boson $A$ as mediator, e.g., the Yukawa suppression stems directly from the Yukawa coupling $\propto y_{f} A \bar{f} f$ (case (ii)), while the isospin suppression arises from the neutralino mixing (case $b$ ): for pure gauge multiplets the coupling $A \bar{\chi} \chi$ would be forbidden by $\mathrm{SU}(2)_{L}$ invariance, and therefore vanishes for $v_{E W} \rightarrow 0$. For a $Z$-boson in the $s$-channel, the discussion of the limit $v_{E W} \rightarrow 0$ is a bit more involved (see appendix A.3), but is essentially analogous to the case of an $A$ mediator.

\subsection{Yukawa and isospin suppression lifting}

In section 2.2, we discussed which three-body final states $\chi \chi \rightarrow B \bar{F} f$ can potentially lift the Yukawa- and/or isospin suppression of the process $\chi \chi \rightarrow \bar{f} f$, for the case in which $B$ is a SM gauge boson or a Higgs boson. This general discussion based on isospin and 


\begin{tabular}{|c|c|c|c|c|c|}
\hline & $Z_{T}$ & $W_{T}$ & $Z_{L} / A$ & $W_{L} / H^{ \pm}$ & $h / H$ \\
\hline \multirow{2}{*}{$\bar{F}_{R} f_{L}$ or $\bar{F}_{L} f_{R}$} & $y_{f} v_{E W}$ & $y_{f} v_{E W}$ & $y_{f}$ & $y_{f}$ & $y_{f}$ \\
& no enhancement & no enhancement & $t / u, s$ & $t / u, s$ & $t / u, s$ \\
\hline \multirow{2}{*}{$\bar{F}_{L} f_{L}$} & 1 & 1 & $v_{E W}$ & $v_{E W}$ & $v_{E W}$ \\
& $t / u(\tilde{B}, \tilde{W}), s(\tilde{H})$ & $t / u(\tilde{B}, \tilde{W}), s(\tilde{H}, \tilde{W})$ & - & $t / u$ & $t / u, s$ \\
\hline \multirow{2}{*}{$\bar{F}_{R} f_{R}$} & 1 & 1 & $v_{E W}$ & $v_{E W}$ & $v_{E W}$ \\
& $t / u(\tilde{B}), s(\tilde{H})$ & - & - & - & $t / u, s$ \\
\hline
\end{tabular}

Table 2. As table 1, but applied to weak gauge and Higgs boson final states within the MSSM. We also indicated whether the process can be realized with the maximal enhancement allowed by chiral and isospin symmetry in $t+u$ and $s$-channel annihilation processes, respectively. For the first two columns we also specify for which neutralino composition $(\tilde{B}=$ bino-like, $\tilde{W}=$ winolike, $\tilde{H}=$ Higgsino-like) the maximal enhancement occurs. For the last three columns $t+u$-channel processes are possible for $\tilde{B}$ - or $\tilde{W}$-like neutralino as well as mixed $\tilde{H} / \tilde{B}$ or $\tilde{H} / \tilde{W}$, and $s$-channel processes are possible for mixed $\tilde{H} / \tilde{B}$ or $\tilde{H} / \tilde{W}$. Entries with a dash do not contribute to the order we are working in (see appendices A.2 and A.3 for details).

chiral symmetry in the limit $v_{E W} \rightarrow 0$ can be extended to the MSSM, as shown in table 2, by noting that all physical Higgs bosons $h, H, A, H^{ \pm}$have isospin $I=1 / 2$. Compared to table 1 , the amplitudes for $A \bar{f} f$ scale as expected in the same way as $Z_{L} \bar{f} f$, noting that in the gauge restoration limit $Z_{L}$ is given by the Goldstone boson $G^{0}$ (and hence transforms in a similar way as the pseudoscalar $A$ ). Similar arguments apply to the other Higgs bosons.

In appendix A.2, we consider the full analytic expressions for six different mass hierarchies of particular phenomenological interest and determine for each of the previously discussed gauge-invariant subsets of diagrams the leading order in $v_{E W}$ and $y_{f}$. The result of this exercise is collected in tables 9-11, where we present the ratio of the leading term for the three-body amplitude and the corresponding two-body amplitude. This allows us, as also indicated in table 2, to identify which contributions to the three-body amplitudes actually realize the suppression lifting that we can maximally expect on the basis of our general symmetry arguments; the 'missing' cases, for which we did not find a contribution within the MSSM, are marked by a '-'. For a detailed technical discussion of the various lifting mechanisms, and how they are realized at the level of individual diagrams, we refer to appendix A.3. We provide a graphical summary in table 3, where we show representative diagrams that realize the lifting of isospin and/or Yukawa suppression, for the sets of gauge invariant classes of diagrams that can be discriminated in the gauge restoration limit (in addition to the three sets discussed before, the $t$-channel can be split into contributions that remain non-zero in the limit of pure neutralino states (I), and those that require neutralino mixing (II)). Isospin suppression can be lifted in all cases by the emission of longitudinal gauge bosons (here represented by the Goldstone bosons) or a Higgs boson. Lifting of Yukawa suppression, as well as lifting of both suppression factors, is more restricted. This can be traced back to basic properties of the unbroken MSSM Lagrangian and the conservation of $J_{C P}=0_{-}$(see appendix A.3 for details), explaining the 'missing' 


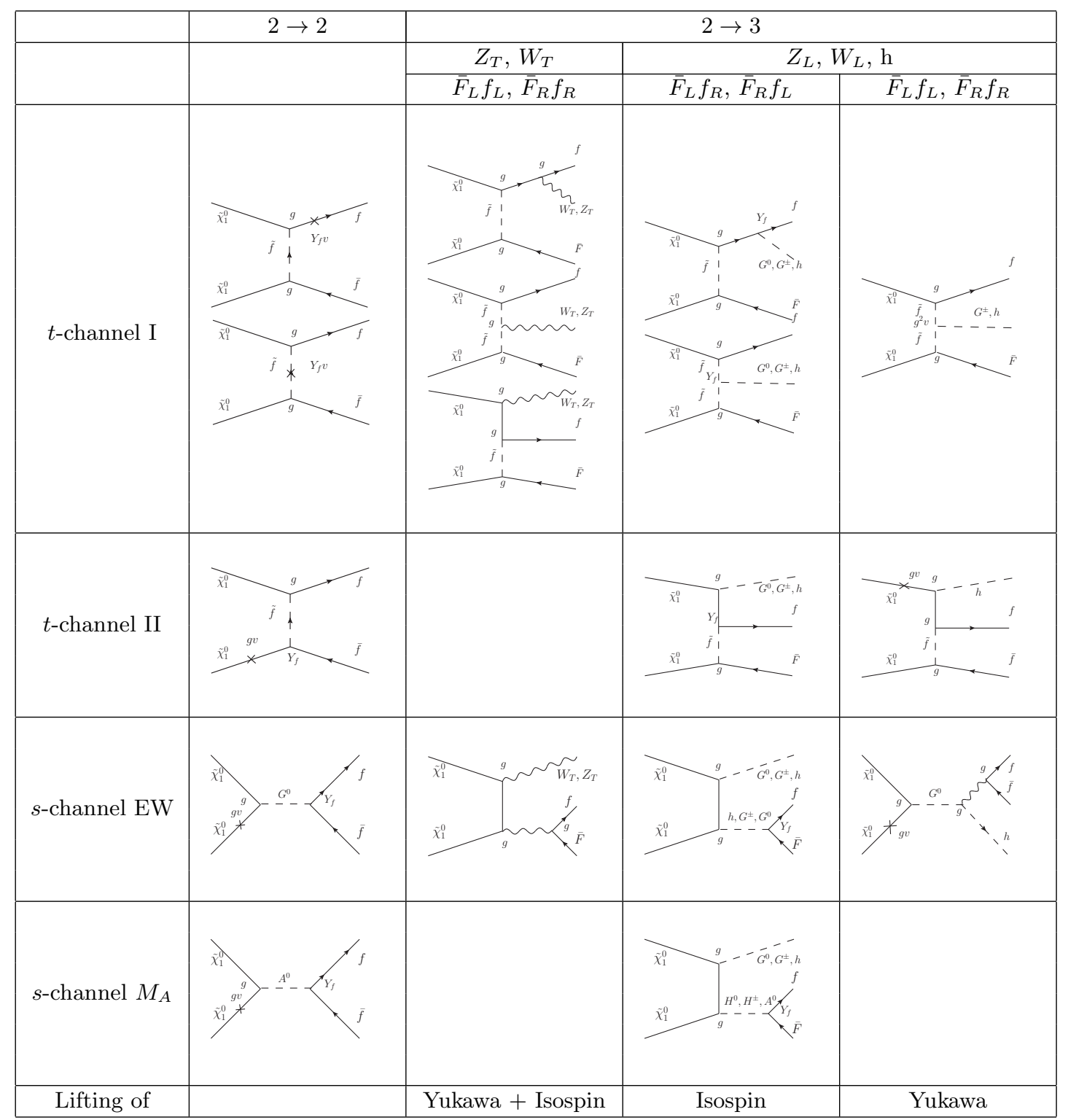

Table 3. Diagrams for annihilation into fermions $\bar{f} f$ and $B \bar{F} f$ (for $B=W, Z, h$ ) in the gauge restoration limit $v_{E W} \rightarrow 0$. The rows correspond to the four gauge-invariant subsets of diagrams that can be discriminated in this limit (see appendix A.3 for details). The first column corresponds to the two-body process, and the other columns show various three-body processes. The diagrams shown in the second column lift both Yukawa and isospin suppression. The diagrams in the third column lift only isospin suppression, and in the fourth column only Yukawa suppression. We show only one representative diagram for each topology (ISR/FSR/VIB) and suppression mechanism. The coupling factors attached to vertices and mass/mixing insertions give the scaling with $y_{f}$, $v_{E W}$ and $g$ of each diagram (for Bino- or Wino-like neutralinos; modifications for Higgsino-like neutralinos are described in appendix A.3). Note that contributions with $W_{T}$ emitted via ISR (second column, first and third row) exist for Wino- or Higgsino-like neutralinos; those with $Z_{T}$ emitted via ISR occur only for a Higgsino-like neutralino. 
entries in table 2. Let us also highlight that the classification procedure revealed ways to lift the two-body suppression that have not been pointed out for the MSSM before (in particular Higgsstrahlung via $t$-channel ISR and a specific $s$-channel VIB process, shown in the last column and second/third row in table 3, respectively).

\subsection{Heavy propagator suppression}

An additional form of suppression, unrelated to the discussion so far, arises in diagrams that rely on mixing between neutralinos or contain heavy propagators. This mass suppression takes the form $\delta_{\mathrm{X}} \equiv m_{\chi} / M_{\mathrm{X}}$, where $\mathrm{X}$ is the heavy state in question. In particular, both $s$-channel contributions to $\chi \chi \rightarrow \bar{f} f$ and a subset of $t$-channel contributions - those of type $(I I)$, see appendix A.2 - rely on mixing the Bino/Wino with the Higgsino. For example, for a Bino- or Wino-like neutralino, the two-body amplitude in the $s$-channel is suppressed by a factor $\delta \mu^{2}=m_{\chi}^{2} / \mu^{2}$ if $|\mu| \gg m_{\chi}$. For a Higgsino-like neutralino, on the other hand, it is suppressed by $\delta_{M_{i}}=m_{\chi} / M_{i}$ for $M_{i} \gg m_{\chi}$, where $M_{i}=\min \left(M_{1}, M_{2}\right)$ (see table 12).

These suppression factors of the $s$-channel annihilation can be lifted for the case of a Wino- or Higgsino-like neutralino by the emission of a (transverse) $W$ or $Z$ from one of the initial neutralino lines (ISR). (The corresponding diagram is illustrated in the third row, second column of table 3.) Additionally, this three-body process simultaneously lifts both isospin- and Yukawa suppression. It is particularly relevant if the two-body final states $W W$ and $Z Z$ are kinematically forbidden, such that the internal gauge boson is off-shell. This is a special case of the threshold effects that we turn to next.

\subsection{Threshold effects}

A given two-body channel $\chi \chi \rightarrow A B$ is strongly phase-space suppressed if the CMS energy is close to the mass of the final-state particles, and for $2 m_{\chi} \leq m_{A}+m_{B}$ the corresponding partial cross section vanishes completely in the $v \rightarrow 0$ limit. If either $A$ or $B$ are off-shell and decay into much lighter states, however, the phase-space opens up again and thereby potentially increases even the total two-body annihilation rate significantly. For the MSSM, this is particularly relevant for the $W^{+} W^{-}$and $\bar{t} t$ channels, which has previously been studied for specific neutralino compositions [46, 47] (for an approximate numerical implementation in the context of relic density calculations, see [48]). For the processes we are interested in here, threshold effects can in general appear for any two-boson final states (or $\bar{t} t$ ).

For a more detailed discussion of this effect, it is useful to rewrite the three-body cross section as (see e.g. $[49,50])$

$$
\begin{aligned}
\sigma v_{2 \rightarrow 3} & =\frac{S}{4 E_{\chi_{1}} E_{\chi_{2}}} \int \overline{\left|\mathcal{M}_{2 \rightarrow 3}\right|^{2}} d \Phi_{3}\left(P ; p_{1}, p_{2}, p_{3}\right) \\
& =\frac{S}{4 E_{\chi_{1}} E_{\chi_{2}}} \int \overline{\left|\mathcal{M}_{2 \rightarrow 3}\right|^{2}} d \Phi_{2}\left(P ; p_{1}, q\right) \times \frac{d q^{2}}{2 \pi} \times d \Phi_{2}\left(q ; p_{2}, p_{3}\right),
\end{aligned}
$$

where $d \Phi_{n}\left(P ; p_{1}, \ldots, p_{n}\right)=(2 \pi)^{4} \delta^{(4)}\left(P-\sum p_{i}\right) \prod_{i} \frac{d^{3} p_{i}}{(2 \pi)^{3} 2 E_{i}}$ is the $n$-body phase space element, $P=p_{\chi_{1}}+p_{\chi_{2}}$ the sum of the 4-momenta of the annihilating neutralinos and $E_{\chi_{i}}$ 
their energy; the $p_{i}$ denote the final-state momenta. Since $q^{2}=\left(p_{2}+p_{3}\right)^{2}$ is time-like, we will in the following often use the notation $q^{2} \equiv m_{23}^{2}$ instead. For the processes considered here, cf. eq. (3.3), the symmetry factor $S$ is always $1 .{ }^{6}$ Furthermore, $\overline{|\mathcal{M}|^{2}}$ denotes the usual squared matrix element, averaged over initial spins and summed over final spins/helicities.

We now assume that the amplitude is dominated by a resonant, almost on-shell internal propagator that decays into particles 2 and 3 , and hence carries momentum $q$. For a resonance $R$ with mass $M$, width $\Gamma$, and spin $1,1 / 2$, or 0 , respectively, we then have

$$
\mathcal{M}_{2 \rightarrow 3}=\frac{1}{m_{23}^{2}-M^{2}+i M \Gamma} \times\left\{\begin{array}{cl}
\mathcal{M}_{2 \rightarrow 2}^{(q) \mu}\left(-g_{\mu \nu}+q_{\mu} q_{\nu} / M^{2}\right) \mathcal{M}_{1 \rightarrow 2}^{(q) \nu} & \text { vector } \\
\mathcal{M}_{2 \rightarrow 2}^{(q)}(\not t+M) \mathcal{M}_{1 \rightarrow 2}^{(q)} & \text { fermion } \\
\mathcal{M}_{2 \rightarrow 2} \mathcal{M}_{1 \rightarrow 2} & \text { scalar }
\end{array}\right.
$$

where $\mathcal{M}_{2 \rightarrow 2}\left(\mathcal{M}_{1 \rightarrow 2}\right)$ is the matrix element for $\chi \chi \rightarrow p_{1} q\left(R^{*} \rightarrow p_{2} p_{3}\right)$, up to polarization vectors or spinors for the 'external' particle $R$ (as indicated by the superscript $q$ ).

The decisive observation is now that $\int\left|\mathcal{M}_{1 \rightarrow 2}\right|^{2} d \Phi_{2}\left(q ; p_{2}, p_{3}\right)$ must be independent of the polarization state of $R$ once all the final state polarizations are summed over. This is familiar from on-shell momenta $q$ - the total (but not differential) decay rate of a particle is independent of its polarization state - but holds more generally for time-like initial momenta $q$ [50]. As long as the full phase-space integral is performed (see section 4.2 for how to treat differential cross sections), one may thus conveniently replace the correlated polarization or spin structure of eq. (3.5) with an unpolarized sum:

$$
\begin{aligned}
\overline{\left|\mathcal{M}_{2 \rightarrow 2}^{(q) \mu}\left(-g_{\mu \nu}+q^{\mu} q^{\nu} / M^{2}\right) \mathcal{M}_{1 \rightarrow 2}^{(q) \nu}\right|^{2}} & =\left|\sum_{\lambda} \mathcal{M}_{2 \rightarrow 2}^{(q) \mu} \epsilon_{\mu}^{* \lambda} \epsilon_{\nu}^{\lambda} \mathcal{M}_{1 \rightarrow 2}^{(q) \nu}\right|^{2} \\
& \rightarrow \frac{1}{3} \sum_{\lambda_{1}, \lambda_{2}}\left|\mathcal{M}_{2 \rightarrow 2}^{(q) \mu} \epsilon_{\mu}^{* \lambda_{1}}\right|^{2}\left|\epsilon_{\nu}^{\lambda_{2}} \mathcal{M}_{1 \rightarrow 2}^{(q) \nu}\right|^{2}, \\
\overline{\left|\mathcal{M}_{2 \rightarrow 2}^{(q)}(q+M) \mathcal{M}_{1 \rightarrow 2}^{(q)}\right|^{2}} & =\left|\sum_{s} \mathcal{M}_{2 \rightarrow 2}^{(q)} u_{s} \bar{u}_{s} \mathcal{M}_{1 \rightarrow 2}^{(q)}\right|^{2} \\
& \rightarrow \frac{1}{2} \sum_{s_{1}, s_{2}}\left|\mathcal{M}_{2 \rightarrow 2}^{(q)} u_{s_{1}}\right|^{2}\left|\bar{u}_{s_{2}} \mathcal{M}_{1 \rightarrow 2}^{(q)}\right|^{2},
\end{aligned}
$$

In this way, we can independently of the spin of $R$ replace

$$
\overline{\left|\mathcal{M}_{2 \rightarrow 3}\right|^{2}} \rightarrow \frac{\overline{\left|\mathcal{M}_{2 \rightarrow 2}\right|^{2}} \overline{\left|\mathcal{M}_{1 \rightarrow 2}\right|^{2}}}{\left(m_{23}^{2}-M^{2}\right)^{2}+M^{2} \Gamma^{2}}
$$

in eq. (3.4) which, for $v \rightarrow 0$, leads to

$$
\sigma v_{2 \rightarrow 3}^{\mathrm{res}}=\mathcal{S} \int_{\left(m_{2}+m_{3}\right)^{2}}^{\left(2 m_{\chi}-m_{1}\right)^{2}} \frac{d m_{23}^{2}}{\pi} \frac{m_{23}}{\left(m_{23}^{2}-M^{2}\right)^{2}+M^{2} \Gamma^{2}} \tilde{\Gamma}_{R \rightarrow 23} \widetilde{\sigma v}_{\chi \chi \rightarrow 1 R} .
$$

\footnotetext{
${ }^{6}$ In general, if some of the final state particles are of identical type, configurations that differ only by exchanging these particles should be counted only once in the phase space integration. Since this will be convenient later on, we thus use a convention where one integrates over all of the phase space as if all particles were distinct, and then correct for the corresponding over-counting by a symmetry factor $S$. It is $S=1$ if all final-state particles are distinct, and $S=1 / 2(S=1 / 6)$ if two (all three) of them are identical.
} 
Here, the decay rate of the off-shell resonance in the frame where $q=\left(m_{23}, \mathbf{0}\right)$ is given by

$$
\tilde{\Gamma}_{R \rightarrow 23} \equiv \frac{S_{23}}{2 m_{23}} \int{\overline{\mid \mathcal{M}_{1 \rightarrow 2}}}^{2} d \Phi_{2}\left(q ; p_{2}, p_{3}\right)=\frac{S_{23}}{16 \pi} \frac{\lambda^{\frac{1}{2}}\left(m_{23}^{2}, m_{2}^{2}, m_{3}^{2}\right)}{m_{23}^{3}}\left|\overline{\mathcal{M}}_{1 \rightarrow 2}\right|^{2},
$$

and the cross section for the annihilation into an off-shell resonance is given by

$$
\widetilde{\sigma v}_{\chi \chi \rightarrow 1 R} \equiv \frac{S_{1 R}}{P^{2}} \int{\overline{\mid \mathcal{M}_{2 \rightarrow 2}}}^{2} d \Phi_{2}\left(P ; p_{1}, q\right)=\frac{S_{1 R}}{128 \pi} \frac{\lambda^{\frac{1}{2}}\left(4 m_{\chi}^{2}, m_{1}^{2}, m_{23}^{2}\right)}{m_{\chi}^{4}}\left|\overline{\mathcal{M}}_{2 \rightarrow 2}\right|^{2} .
$$

In the last step we performed the phase-space integral explicitly by using the fact that for $v \rightarrow 0$ the annihilation process is kinematically the same as a pseudo-scalar decay, implying that $\overline{\mid \mathcal{M}}^{2}$ cannot have any angular dependence. ${ }^{7}$ Eq. (3.9) will thus continue to hold for general $s$-wave annihilation, provided one replaces $4 m_{\chi} \rightarrow s$ in eq. (3.11). The squared matrix elements are here again summed (averaged) over final (initial) spins/helicities, leading to an overall symmetry factor of $\mathcal{S}=S /\left(S_{1 R} S_{23}\right)$ (with $S_{1 R}, S_{23}$ defined in accordance with footnote 6$)$.

We note that eq. (3.9) can be significantly simplified by a few well-motivated assumptions. Concretely, let us assume the off-shell particle to decay to massless final states, $m_{2}=$ $m_{3}=0$, and ${\overline{\left|\mathcal{M}_{1 \rightarrow 2}\right|}}^{2} \propto M^{2}$ close to the threshold; this implies $\tilde{\Gamma}_{R \rightarrow 23}=\left(m_{23} / M\right) \Gamma_{R \rightarrow 23}$. We also introduce a reduced cross section

$$
(\sigma v)_{\mathrm{red}} \equiv(\sigma v)_{\chi \chi \rightarrow 1 R} / \lambda^{n+1 / 2}\left(1, \mu_{1}, \mu_{R}\right)
$$

with $\mu_{R} \equiv m_{23}^{2} / s$ and $\mu_{1} \equiv m_{1}^{2} / s$, allowing for the two-body cross section close to threshold to be suppressed not only by a phase-space factor $(n=0)$, but by an additional such factor from the matrix element itself (as e.g. in the example of Higgsino annihilation below, for which we have $n=1)$. By definition, $(\sigma v)_{\text {red }}$ thus remains finite both above and below the threshold. Assuming $(\sigma v)_{\text {red }}$ to be independent of $m_{23}$ close to threshold, eq. (3.9) simplifies to

$$
\sigma v_{\chi \chi \rightarrow 1 R^{*}} \simeq \mathcal{S}(\sigma v)_{\mathrm{red}} \int_{0}^{\mu_{\max }} \frac{d \mu}{\pi} \frac{\gamma \mu}{(\mu-1)^{2}+\gamma^{2}} \lambda^{n+1 / 2}\left(1, \mu_{1}, \mu \mu_{R}\right)
$$

where $\mu_{\max }=\left(\sqrt{s}-m_{1}\right)^{2} / m_{R}^{2}$ and $\gamma \equiv \Gamma_{R} / M$. This expression is model-independent in the sense that the threshold correction can be directly estimated for any given two-body cross section (i.e. without first having to compute $\tilde{\sigma v}$ or $\tilde{\Gamma}$ ).

As an illustrative and concrete example, let us consider the process $\chi \chi \rightarrow W^{-} e^{+} \nu_{e}$ in the limit of pure Higgsino DM. For simplicity, we assume that sleptons are much heavier

\footnotetext{
${ }^{7}$ For this reason, the result takes the same form as for off-shell decays [51, 52], suggesting a straightforward generalization to 4-body final states dominated by the annihilation into two off-shell particles:

$$
\sigma v_{2 \rightarrow 4}^{\mathrm{res}}=\mathcal{S} \int \frac{d m_{12}^{2}}{\pi} \frac{d m_{34}^{2}}{\pi} \frac{m_{12}}{\left(m_{12}^{2}-M_{R_{1}}^{2}\right)^{2}+M_{R_{1}}^{2} \Gamma_{R_{1}}^{2}} \frac{m_{23}}{\left(m_{23}^{2}-M_{R_{2}}^{2}\right)^{2}+M_{R_{2}}^{2} \Gamma_{R_{2}}^{2}} \tilde{\Gamma}_{R_{1} \rightarrow 12} \tilde{\Gamma}_{R_{2} \rightarrow 34} \widetilde{\sigma v}_{\chi \chi} \rightarrow R_{1} R_{2} .
$$
}




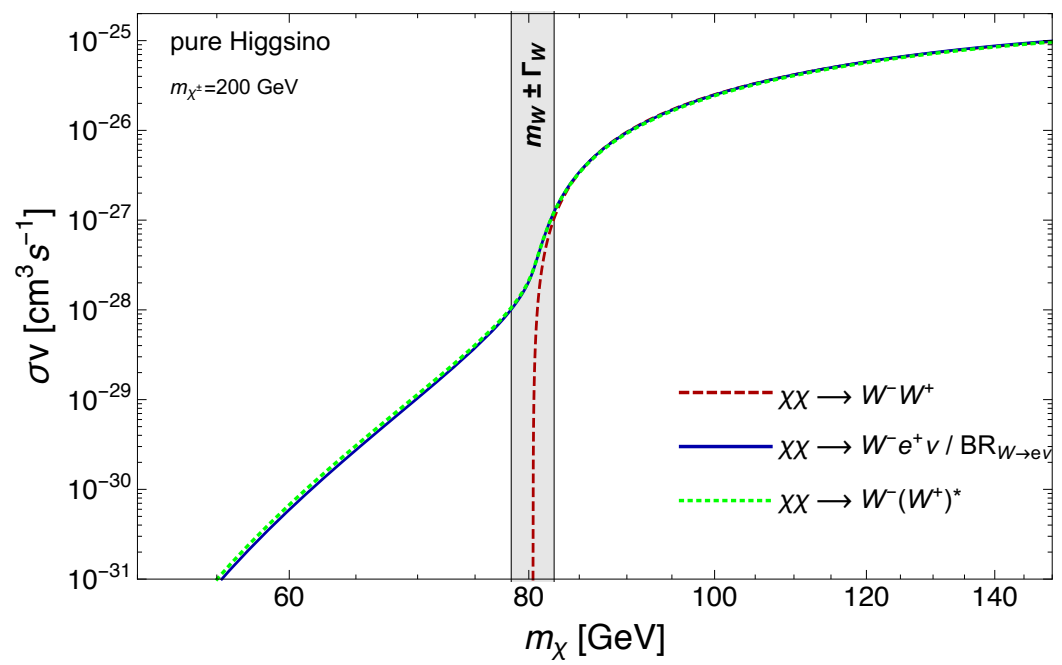

Figure 5. Cross section for pure Higgsinos annihilating to $W$ bosons, $\chi \chi \rightarrow W^{+} W^{-}$, compared to $\chi \chi \rightarrow W^{-} e^{+} \nu$. For the latter process, we show the cross section divided by the branching fraction $\Gamma_{W \rightarrow e \nu} / \Gamma_{W} \simeq 1 / 9$ (solid lines). For comparison, we also include the model-independent estimate of eq. (3.14) for $\chi \chi \rightarrow W^{-}\left(W^{+}\right)^{*}$ (dotted lines). For $m_{\chi} \lesssim m_{W}$, the three-body cross section is clearly larger than the lowest-order result; above the threshold, on the other hand, the two agree exactly.

than the neutralino, such that the only contributing diagrams are of the $V=W^{-}$ISR type, with a virtual Higgsino-like chargino and a resonance $R=W^{+*}$. In this limit, we find

$$
\widetilde{\sigma v}_{\chi \chi \rightarrow W W^{*}}=\frac{g^{4}}{512 \pi} \frac{\left(16 m_{\chi}^{4}-8 m_{\chi}^{2}\left(m_{W}^{2}+m_{23}^{2}\right)+\left(m_{W}^{2}-m_{23}^{2}\right)^{2}\right)^{\frac{3}{2}}}{\left(2 m_{\chi}^{2}+2 m_{\tilde{\chi}^{+}}^{2}-m_{W}^{2}-m_{23}^{2}\right)^{2}}
$$

and

$$
\tilde{\Gamma}_{W^{*} \rightarrow e \nu}=\frac{g^{2} m_{23}}{48 \pi}=\frac{m_{23}}{m_{W}} \Gamma_{W \rightarrow e \nu} .
$$

We calculate the full three-body cross section as derived in appendix A.1, in the pure Higgsino limit, and then compare it to the result given in eq. (3.9). As shown in figure 5, we obtain excellent agreement even though both the directly involved amplitudes and the numerical phase-space integrations are very different in nature (the two results for the three-body cross section, shown as solid lines, lie exactly on top of each other). This should of course be expected for a process which by construction only receives contributions from an off-shell final-state particle, but we stress that eq. (3.9) is in general much simpler to calculate in praxis for such cases. For comparison, we also indicate (with dotted lines) the model-independent result given in eq. (3.14); as one can see, even this simplified expression provides an excellent approximation to the full result.

Most importantly, our example illustrates the much more general point that a threebody process around or below the kinematic threshold of a large two-body process can be significantly enhanced over the total annihilation rate at lowest order. Above the threshold and rescaled to the relevant branching ratio for the decay of the resonance $R$, on the other 
hand, the three-body cross section for a process $\chi \chi \rightarrow 1 R, R \rightarrow 23$ equals almost exactly the two-body result - an effect which we will discuss in detail in the next section.

\section{Double counting issues}

We now turn to double counting issues related to unstable final-state particles. If the final state of a two-body annihilation process undergoes a subsequent $1 \rightarrow 2$ decay, in particular, this can also be viewed as a three-body process with the unstable particle (the resonance, in our wording) as an intermediate state. While we discussed the situation below the kinematic threshold for the production of the unstable particle in section 3.5 as a way of enhancing the total cross section, we are here interested in the kinematic region above the threshold. As before, this is relevant for all massive diboson as well as $\bar{t} t$ final states considered here.

One possibility to avoid over-counting identical kinematic configurations when adding two-body and three-body processes would be to altogether disregard the former for massive diboson or $\bar{t} t$ final states. Interferences between (nearly) on- and off-shell contributions to the amplitude would then be correctly accounted for, as well as the impact of the spin of the resonance. However, this procedure has several drawbacks on a practical level, and furthermore turns out to be incorrect for two-body processes with identical particles in the final state (such as e.g. $\chi \chi \rightarrow Z Z$ ), as will be discussed in more detail below. We therefore prefer to explicitly subtract on-shell contributions to the three-body processes, which allows us to keep most of the advantages of the full three-body computation while correctly taking into account all symmetry factors. In the following we describe this procedure in more detail for both the total cross section and the differential yield of e.g. gamma rays.

\subsection{Narrow width approximation and total cross section}

For three-body processes dominated by an on- or off-shell resonance, the total cross section can be written as in eq. (3.9). If the intermediate particle corresponds to a nearly on-shell resonance with $\Gamma \ll M$, furthermore, the Breit-Wigner propagator can be approximated as

$$
\frac{1}{\left(m_{23}^{2}-M^{2}\right)^{2}+M^{2} \Gamma^{2}} \rightarrow \frac{\pi}{M \Gamma} \delta\left(m_{23}^{2}-M^{2}\right) .
$$

This narrow-width approximation (NWA) yields the on-shell contribution of the resonance $R$, and we denote the corresponding, approximated cross section by $\sigma v^{N W A}$. Strictly speaking, for the approximation to work well, the kinematic boundaries have to be sufficiently far away from the pole, $\left|m_{23}^{2}-M^{2}\right| \gg M \Gamma$, and all contributions from the matrix element and phase-space factors apart from the Breit-Wigner propagator should be smooth functions of $m_{23}^{2}$ in the vicinity of the pole, which we assume in the following. With this replacement in eq. (3.9), we immediately recover the well-known result

$$
\sigma v_{2 \rightarrow 3}^{N W A}=\mathcal{S} \times \sigma v_{\chi \chi \rightarrow 1, R} \times B R_{R \rightarrow 23}
$$

where $B R_{R \rightarrow 23}=\Gamma_{R \rightarrow 23} / \Gamma$ is the branching ratio for the resonance $R$ to decay into particles 2 and $3, \Gamma_{R \rightarrow 23}=S_{1 \rightarrow 2} /(2 M) \int d \Phi_{2} \overline{\left|\mathcal{M}_{d}\right|^{2}}$ is the partial decay width, and $\sigma v_{\chi \chi \rightarrow 1 R}=$ $S_{2 \rightarrow 2} / P^{2} \int d \Phi_{2} \overline{\left|\mathcal{M}_{p}\right|^{2}}$ is the two-body cross section. 
In general, more than one resonance can contribute to a given three-body process, and one has to sum over all those contributions (in principle there can be interference effects for overlapping resonances with $\left|M_{1}-M_{2}\right| \lesssim \Gamma_{1}+\Gamma_{2}$; we will assume this is not the case). The narrow-width limit for the processes in eq. (3.3) is thus given by

$$
\begin{aligned}
\sigma v_{W^{+} f \bar{F}}^{N W A}= & \sigma v_{W W} B R(W \rightarrow f \bar{F})+\sigma v_{W^{+} H^{-}} B R\left(H^{-} \rightarrow f \bar{F}\right) \\
\sigma v_{W+b \bar{t}}^{N W A}= & \sigma v_{W^{+} H^{-}} B R\left(H^{-} \rightarrow b \bar{t}\right)+\sigma v_{t \bar{t}} B R\left(t \rightarrow W^{+} b\right) \\
\sigma v_{Z f \bar{f}}^{N W A}= & 2 \sigma v_{Z Z} B R(Z \rightarrow f \bar{f})+\sigma v_{Z H} B R(H \rightarrow f \bar{f}) \\
& +\sigma v_{Z h} B R(h \rightarrow f \bar{f}) \\
\sigma v_{H^{+} f \bar{F}}^{N W A}= & \sigma v_{W^{-} H^{+}} B R(W \rightarrow f \bar{F}) \\
\sigma v_{A f \bar{f}}^{N W A}= & \sigma v_{A h} B R(h \rightarrow f \bar{f})+\sigma v_{A H} B R(H \rightarrow f \bar{f}) \\
\sigma v_{H f \bar{f}}^{N W A}= & \sigma v_{A H} B R(A \rightarrow f \bar{f})+\sigma v_{Z H} B R(Z \rightarrow f \bar{f}) \\
\sigma v_{h f \bar{f}}^{N W A}= & \sigma v_{A h} B R(A \rightarrow f \bar{f})+\sigma v_{Z h} B R(Z \rightarrow f \bar{f}),
\end{aligned}
$$

where $f$ denotes any SM fermion, and $F$ its $\mathrm{SU}(2)_{L}$ doublet partner. The branching fractions $B R$ are given by the tree-level decay widths, divided by the total width appearing in the corresponding Breit-Wigner propagators. As stated in the second line, third-generation quarks have to be treated separately because of the contribution from top decay. Note that these results justify why the interference effects mentioned above can indeed be neglected: those would be potentially relevant only in small regions of the MSSM parameter space, where the charged Higgs is degenerate in mass with the $W$ boson or the top quark (or, instead, one of the heavy neutral Higgses close in mass to the $Z$ boson or the SM Higgs $h$ ).

The total annihilation cross section is then given by

$$
\sigma v=\sigma v_{2 \rightarrow 2}+\sigma v_{2 \rightarrow 3}-\sigma v_{2 \rightarrow 3}^{N W A},
$$

where each term corresponds to the sum over all possible 2- and three-body final states, respectively. ${ }^{8}$ In the following, we refer to the difference $\sigma v_{2 \rightarrow 3}^{\text {sub }} \equiv \sigma v_{2 \rightarrow 3}-\sigma v_{2 \rightarrow 3}^{N W A}$ as the (NWA-subtracted) contribution from three-body final states, with a similar definition for individual three-body final states. We note that $\sigma v_{2 \rightarrow 3}^{\text {sub }}$ can be negative (although $\sigma v>0$, of course). To match our conventions for the computation of three-body cross sections, the two-body cross sections appearing above should be evaluated in $s$-wave approximation. Finally, we stress that, even when summing over all possible three-body final states, $\sigma v_{2 \rightarrow 3}^{N W A}$ is not equal to the sum over two-body cross sections with diboson and $t \bar{t}$ final states, as

\footnotetext{
${ }^{8}$ We neglect loop corrections to $\sigma v_{2 \rightarrow 2}$ because only three-body final states can lift the $m_{f}^{2} / m_{\chi}^{2}$ suppression. For very heavy neutralinos, however, enhancements $\propto \frac{\alpha}{\pi} \ln ^{2}\left(m_{W}^{2} / m_{\chi}^{2}\right)$ from both soft/collinear IB and one-loop corrections to $\sigma v_{2 \rightarrow 2}$ can become sizeable. For EW corrections these logarithmic terms will in general give a non-zero contribution, unless the initial state is a singlet under $\mathrm{SU}(2)_{L} \times \mathrm{U}(1)_{Y}$, such as for a pure Bino [53, 54] (the latter also applies to U(1) and SU(3) IB; see ref. [29] for an efficient modelindependent way of taking the relevant loop contributions into account). Resummation of logarithmically enhanced contributions has been discussed e.g. in [55-57] for pure Winos and Higgsinos. In addition, for neutralinos with a significant Wino fraction and TeV mass, Sommerfeld enhancement can play an important role [58-60]. A joint treatment of all these effects for the general MSSM is beyond the scope of this work, but would be desirable in view of future indirect detection probes.
} 
one may have naively expected. This is partially because the Higgs resonances can also decay into pairs of bosons, and partially due to a mismatch in the combinatorial factors, which can be traced back to ambiguities in the narrow-width limit. For example, for $\chi \chi \rightarrow Z Z^{*} \rightarrow Z f \bar{f}$, each of the $Z$ bosons could act as intermediate resonance, which intuitively explains the factor $\mathcal{S}=2$ encountered in this case. These ambiguities would disappear if one were to treat both $Z$ bosons on equal footing, i.e. consider 4-body final states, which however is impractical for a general MSSM computation.

\subsection{Spectrum of stable particles}

In general, our final state particles $p$ will fragment and decay into potentially observable particles $P$, such as gamma rays or antiprotons. For a given three-body annihilation channel, and a conventional normalization to the corresponding yield from the two-body rate, the spectrum can be written as

$$
\frac{d N_{P}}{d E_{P}}=\frac{1}{\sigma v_{2 \rightarrow 2}^{\text {tot }}} \sum_{p} \int_{E_{p}^{\min }}^{E_{p}^{\max }} \frac{d N_{P}^{p \rightarrow P+\ldots}}{d E_{P}} \frac{d \sigma v_{2 \rightarrow 3}}{d E_{p}} d E_{p} .
$$

Here, $d N_{P}^{p \rightarrow P+\ldots /} / d E_{P}$ describes the number of stable particles $P$, per energy bin, that result from the inclusive process $p \rightarrow P+\ldots$ of a particle $p$ decaying in flight (with energy $E_{p}$ ), and the sum has in principle to be performed over all helicity states separately (because $d N / d E_{P}$ can differ for different helicities of $p$ ). Assuming $C P$ conservation and that the decaying particles have very narrow widths, a very useful approximation in practice consists in consid-

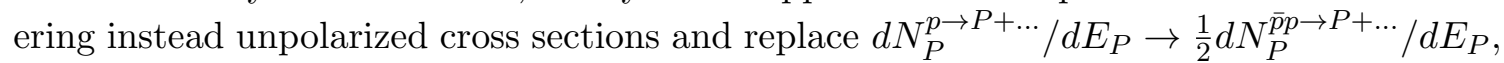
where the inclusive process $\bar{p} p \rightarrow P+\ldots$ is evaluated for a CMS energy of $2 E_{p}$ (see e.g. [18]). This quantity can easily be obtained from event generators like Pythia [61] and, unlike $d N_{P}^{p \rightarrow P+\ldots} / d E_{P}$, has the further advantage of being manifestly color neutral. ${ }^{9}$

The above expression depends on the differential cross section $d \sigma / d E_{p}$, rather than the total cross section discussed in the previous subsection, implying that we need to re-discuss how to correctly take into account double-counting issues. Consider for example the process $\chi \chi \rightarrow H^{+} f \bar{F}$. We want to remove the contribution already contained in $\chi \chi \rightarrow H^{+} W^{-}$, say in the differential cross section for the fermion $f$,

$$
\left(\frac{d \sigma v_{H^{+} f \bar{F}}}{d E_{f}}\right)^{s u b}=\frac{d \sigma v_{H^{+} f \bar{F}}}{d E_{f}}-\left(\frac{d \sigma v_{H^{+} f \bar{F}}}{d E_{f}}\right)^{N W A} .
$$

\footnotetext{
${ }^{9}$ Note that for quark final states eq. (4.11) is not correct beyond leading order, where partons fragment independently, because it ignores flux tubes. In general, for a final state consisting of a (color-neutral) boson $B$ and a quark pair $\bar{q} q$, which may have different masses, one should instead consider

$$
\sigma v_{2 \rightarrow 2}^{\text {tot }} \frac{d N_{P}}{d E_{P}}=\int_{E_{B}^{\min }}^{E_{B}^{\max }} \frac{d N_{P}^{B \rightarrow P+\cdots}}{d E_{P}} \frac{d \sigma v_{2 \rightarrow 3}}{d E_{B}} d E_{B}+\int_{E_{\bar{q}}^{\min }}^{E_{\bar{q}}^{\max }} \int_{E_{q}^{\min }}^{E_{q}^{\max }} \frac{d N_{P}^{\bar{q} q \rightarrow P+\cdots}}{d E_{P}} \frac{d \sigma v_{2 \rightarrow 3}}{d E_{\bar{q}} d E_{q}} d E_{\bar{q}} d E_{q} .
$$

The fragmentation function $d N_{P}^{\bar{q} q \rightarrow P+\ldots} / d E_{P}$ that appears here can be obtained by boosting to the backto-back system of the quarks, defined as $\mathbf{p}_{q}^{\mathrm{bb}}=-\mathbf{p}_{\bar{q}}^{\mathrm{bb}}$, then evaluate the fragmentation function supplied by, e.g., Pythia for a CMS energy of $E_{q}^{\mathrm{bb}}+E_{\bar{q}}^{\mathrm{bb}}$, and finally boosting back to the DM frame. As we only consider leading order effects here, and the expected difference in $d N_{P} / d E_{P}$ is anyway very small, we restrict our numerical implementation to eq. (4.11) also for quark final states.
} 
The question is, what to use for the NWA term. The simplest assumption would be to replace the branching ratio in eq. (4.2) by the differential spectrum, i.e.

$$
\left(\frac{d \sigma v_{H^{+} f \bar{F}}}{d E_{f}}\right)^{N W A}=\sigma v_{H^{+} W^{-}} \frac{d N_{W^{-} \rightarrow f \bar{F}}}{d E_{f}}
$$

where the last factor is the spectrum of $f$ per decay of the $W$, as seen in the CMS frame and normalized such that $\int d E_{f} d N_{W} / d E_{f}=B R(W \rightarrow f \bar{F})$. Obviously, it is straightforward to generalize eqs. (4.13), (4.14) to other final states, analogous to eqs. (4.3)-(4.9).

Unlike for the total cross section [50], however, the replacements (3.6) for vector and fermion resonances are in general not correct for the differential cross section. Instead, the latter can be affected by the correlation of the helicities/spins of the resonance between the production and decay processes, even in the limit $\Gamma / M \rightarrow 0$. Fortunately, conservation of $C P$ and total angular momentum uniquely fixes the polarization states of the vector resonances for the case of Majorana pair annihilation in the $s$-wave limit (see, e.g., [62]): vector bosons in $W W$ and $Z Z$ final states are necessarily transversely polarized, and those in $H^{ \pm} W^{\mp}, H Z$ and $h Z$ final states longitudinal (while $A Z$ final states are not possible, for the same reason). Therefore, spin correlations can fully be taken into account by using the appropriate energy spectra for polarized vector bosons in eq. (4.14), noting that the branching fractions are in fact independent of polarization (see appendix $\mathrm{C}$ for a more detailed discussion). For the example above, this implies that one should use $d N_{W_{L} \rightarrow f \bar{F}} / d E_{f}$ instead of $d N_{W \rightarrow f \bar{F}} / d E_{f}$; for e.g. $\chi \chi \rightarrow W W^{*} \rightarrow W f \bar{F}$, on the other hand, the corresponding narrow-width contribution contains $d N_{W_{T} \rightarrow f \bar{F}} / d E_{f}$. For the decay of a top resonance, $\chi \chi \rightarrow t^{*} \bar{t} \rightarrow W^{+} b \bar{t}$, conservation of angular momentum and $C P$ requires $t^{*}$ and $\bar{t}$ to have the same helicity. We note that the decay spectrum for polarized tops, $d N_{t_{h} \rightarrow W^{+} b} / d E_{b}$, differs for the two helicities $h= \pm 1 / 2$, due to the parity-violating $W$ coupling. Nevertheless, since both polarizations are produced with equal cross section, as a consequence of $C P$ conservation, the relevant contribution to the total narrow-width spectrum is given by

$$
\sum_{h= \pm 1 / 2} \sigma v_{t_{h} \bar{t}_{h}} \frac{d N_{t_{h} \rightarrow W^{+} b}}{d E_{b}}=\frac{\sigma v_{t \bar{t}}}{2}\left(\frac{d N_{t_{1 / 2} \rightarrow W^{+} b}}{d E_{b}}+\frac{d N_{t_{-1 / 2} \rightarrow W^{+} b}}{d E_{b}}\right)=\sigma v_{t \bar{t}} \frac{d N_{t \rightarrow W^{+} b}}{d E_{b}}
$$

where $\sigma v_{t \bar{t}} / 2$ is the usual cross section summed over all final-state helicities. Thus, also for top resonances, no polarization effects occur in the CP conserving MSSM.

In summary, the differential three-body cross section to be used in eq. (4.11) is given by

$$
\left(\frac{d \sigma v_{2 \rightarrow 3}}{d E_{p}}\right)^{\mathrm{sub}} \equiv \frac{d \sigma v_{2 \rightarrow 3}}{d E_{p}}-\left(\frac{d \sigma v_{2 \rightarrow 3}}{d E_{p}}\right)^{\mathrm{NWA}}
$$

where for fermionic final state particles $(p=f, \bar{f}, F, \bar{F})$ we have

$$
\begin{aligned}
\left(\frac{d \sigma v_{W^{+} f \bar{F}}}{d E_{p}}\right)^{\mathrm{NWA}} & =\sigma v_{W W} \frac{d N_{W_{T} \rightarrow f \bar{F}}}{d E_{p}}+\sigma v_{W^{+} H^{-}} \frac{d N_{H^{-} \rightarrow f \bar{F}}}{d E_{p}} \\
\left(\frac{d \sigma v_{Z f \bar{f}}}{d E_{p}}\right)^{\mathrm{NWA}} & =2 \sigma v_{Z Z} \frac{d N_{Z_{T} \rightarrow f \bar{f}}}{d E_{p}}+\sigma v_{Z H} \frac{d N_{H \rightarrow f \bar{f}}}{d E_{p}}+\sigma v_{Z h} \frac{d N_{h \rightarrow f \bar{f}}}{d E_{p}}
\end{aligned}
$$




$$
\begin{aligned}
\left(\frac{d \sigma v_{H^{+} f \bar{F}}}{d E_{p}}\right)^{\mathrm{NWA}} & =\sigma v_{W^{-} H^{+}} \frac{d N_{W_{L} \rightarrow f \bar{F}}}{d E_{p}} \\
\left(\frac{d \sigma v_{A f \bar{f}}}{d E_{p}}\right)^{\mathrm{NWA}} & =\sigma v_{A h} \frac{d N_{h \rightarrow f \bar{f}}}{d E_{p}}+\sigma v_{A H} \frac{d N_{H \rightarrow f \bar{f}}}{d E_{p}} \\
\left(\frac{d \sigma v_{H f \bar{f}}}{d E_{p}}\right)^{\mathrm{NWA}} & =\sigma v_{A H} \frac{d N_{A \rightarrow f \bar{f}}}{d E_{p}}+\sigma v_{Z H} \frac{d N_{Z_{L} \rightarrow f \bar{f}}}{d E_{p}} \\
\left(\frac{d \sigma v_{h f \bar{f}}}{d E_{p}}\right)^{\mathrm{NWA}} & =\sigma v_{A h} \frac{d N_{A \rightarrow f \bar{f}}}{d E_{p}}+\sigma v_{Z h} \frac{d N_{Z_{L} \rightarrow f \bar{f}}}{d E_{p}} .
\end{aligned}
$$

Here, the decay spectra are normalized to fermionic branching ratios, e.g. $\int d E_{f} d N_{W_{\lambda} \rightarrow f \bar{F}} / d E_{f}=B R(W \rightarrow f \bar{F})$. For scalars, or when neglecting correlations, these spectra are flat, e.g.

$$
\frac{d N_{h \rightarrow b \bar{b}}}{d E_{b}}=\frac{B R(h \rightarrow b \bar{b})}{E_{b}^{\max }-E_{b}^{\min }},
$$

where $E_{b}^{\max / \min }$ are the maximal and minimal allowed energy of the $b$ in the decay of the (boosted) Higgs. This is the usual box-shaped spectrum in a cascade decay. The non-trivial spectra are in principle straight-forward to derive (see appendix C), but not needed in our numerical implementation (see below). For bosonic final states $\left(p=Z, W^{ \pm}, h, H, H^{ \pm}, A\right)$, on the other hand, there is no polarization effect and only the energy allowed by the two-body kinematics contributes. This implies that one has to replace

$$
\sigma v_{p X} \frac{d N_{X_{(h)} \rightarrow f \bar{F}}}{d E_{p}} \longrightarrow \sigma v_{p X} B R(X \rightarrow f \bar{F}) \delta\left(E_{p}-E_{p}^{\chi \chi \rightarrow p X}\right)
$$

in eqs. (4.17)-(4.22), where $E_{p}^{\chi \chi \rightarrow p X}=m_{\chi}+\left(m_{p}^{2}-m_{X}^{2}\right) / 4 m_{\chi}$. Annihilation channels involving top quarks, finally, are slightly special:

$$
\begin{aligned}
& \left(\frac{d \sigma v_{W^{+} b \bar{t}}}{d E_{b}}\right)^{\mathrm{NWA}}=\sigma v_{W^{+} H^{-}} \frac{d N_{H^{-} \rightarrow t \bar{b}}}{d E_{b}}+\sigma v_{\bar{t} t} \frac{d N_{t \rightarrow W^{+} b}}{d E_{b}} \\
& \left(\frac{d \sigma v_{W^{+} b \bar{t}}}{d E_{\bar{t}}}\right)^{\mathrm{NWA}}=\sigma v_{W^{+} H^{-}} \frac{d N_{H^{-} \rightarrow t \bar{b}}}{d E_{\bar{t}}}+\sigma v_{\overline{t t}} B R(t \rightarrow b W) \delta\left(E_{\bar{t}}-m_{\chi}\right) \\
& \left(\frac{d \sigma v_{W^{+} b \bar{t}}}{d E_{W}}\right)^{\mathrm{NWA}}=\sigma v_{\bar{t} t} \frac{d N_{t \rightarrow W^{+} b}}{d E_{W}}+\sigma v_{W^{+} H^{-}} B R\left(H^{-} \rightarrow \bar{t} b\right) \delta\left(E_{W}-E_{W}^{\chi \chi \rightarrow W^{+} H^{-}}\right)
\end{aligned}
$$

Now let us consider these NWA corrections to the energy distribution of final state particles $p$ in the context of eq. (4.11), i.e. the spectrum of stable particles $P$. From each of the terms on the r.h.s. of eqs. (4.17)-(4.22), and a given channel $\chi \chi \rightarrow Y f \bar{F}$, we pick up a contribution of the form

$$
\begin{gathered}
\frac{\sigma v_{Y X}}{\sigma v_{2 \rightarrow 2}^{\text {tot }}}\left\{\sum_{p=f, \bar{F}} \int \frac{d N_{P}^{p \rightarrow P+\ldots}}{d E_{P}} \frac{d N_{X_{(h) \rightarrow f \bar{F}}}}{d E_{p}} d E_{p}+\left.B R(X \rightarrow f \bar{F}) \frac{d N_{P}^{Y \rightarrow P+\ldots}}{d E_{P}}\right|_{E_{Y}=E_{Y}^{\chi \chi \rightarrow Y X}}\right\} \\
\quad=B R(\chi \chi \rightarrow Y X)\left\{\frac{d N_{P}^{X}(h) \rightarrow f \bar{F} \rightarrow P+\ldots}{d E_{P}}+B R(X \rightarrow f \bar{F}) \frac{d N_{P}^{Y \rightarrow P+\ldots}}{d E_{P}}\right\}_{E_{X, Y}=E_{X, Y}^{\chi \chi \rightarrow Y X}}
\end{gathered}
$$




$$
\begin{aligned}
& \rightarrow B R(\chi \chi \rightarrow Y X) B R(X \rightarrow f \bar{F})\left\{\frac{d \hat{N}_{P}^{X}(h) \rightarrow P+\ldots}{d E_{P}}+\frac{d N_{P}^{Y \rightarrow P+\ldots}}{d E_{P}}\right\}_{E_{X, Y}=E_{X, Y}^{\chi \chi \rightarrow Y X}} \\
& =B R(\chi \chi \rightarrow Y X) B R(X \rightarrow f \bar{F}) \frac{d \hat{N}_{P}^{X Y \rightarrow P+\ldots}}{d E_{P}} .
\end{aligned}
$$

Here, we can replace $X_{(h)} \rightarrow X$ in the last step because, as stressed before, the helicity of $X$ in $\chi \chi \rightarrow X Y$ is uniquely fixed by conservation of angular momentum and $C P$ (for $Y$, on the other hand, the helicity has been fixed that way right from the start). Furthermore, we introduced the notation $d \hat{N}_{P}^{X(h) \rightarrow P+\ldots} / d E_{P}$ for the yield of species $P$ considering only

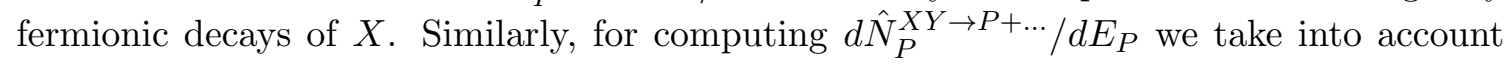
only fermionic decays of $X$ (while for $Y$ all decay modes are included). Note that the second step $(\rightarrow)$ is then only valid under the assumption that $d N / d E_{P}$ has the same shape for a single decay channel $X_{(h)} \rightarrow f \bar{F}$ as for the sum over all fermionic final states, which can be a rather poor approximation for a given channel (involving, e.g., neutrinos). The total spectrum of a given stable state particle $P$, however, is correctly recovered when summing eq. (4.30) over all possible three-body channels involving the boson $Y$ and a pair of fermions. Numerically, we implement this sum for all fermionic final states for decays of $X=$ $Z, W, h, H, H^{ \pm}, A$. For cases where a nearly on-shell $t^{*}$ quark gives a large contribution, even the single-channel yield is well approximated because the decay $t \rightarrow W b$ dominates.

Let us conclude this section with two comments regarding the correct use of final state helicities for the determination of yields of stable particles. i) eq. (4.11) has indeed to be summed over all helicities of $p$ that contribute to the cross section $\sigma_{2 \rightarrow 3}$; it is thus in general

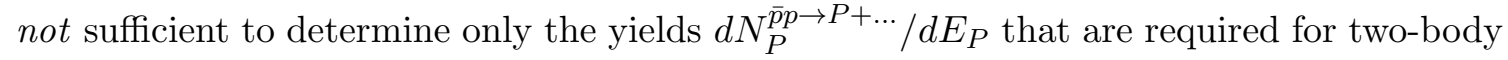
processes (for which the helicities of $p$ and $\bar{p}$ are fixed by the requirement $J^{P}=0^{-}$). ii) The yields from the NWA subtraction, on the other hand, do result from final states with helicities fixed by the same symmetry argument as in the two-body case. This implies that double counting is fully avoided in our prescription even if the yields $d N_{P}^{X Y \rightarrow P+\ldots / d E_{P}}$ are throughout approximated by using unpolarized final state particles $X$ and $Y$ : in that case, the procedure described above consistently removes the double counting related to the yields produced from decay and fragmentation of $Y$. For $X$, on the other hand, the full three-body matrix element automatically takes into account polarization effects in the decay $X \rightarrow f \bar{F}$, while the NWA term subtracts the yield for unpolarized decays. This means that, in this case, the NWA-subtracted three-body contribution accounts precisely for the difference, and correctly replaces the unpolarized by the polarized yield for the $X$ decay after adding two- and three-body contributions.

\section{Results for the MSSM}

In order to demonstrate the impact of our results on realistic models, we work in the framework of simplified phenomenological MSSM versions, introduced in section 5.1, that are however generic enough to capture the relevant phenomenology discussed in this work. We assess in turn the consequences for the overall annihilation cross section (section 5.2) and yields (section 5.3), before discussing in detail selected example spectra in section 5.4. 


\begin{tabular}{|c|c|c|c|c|c|c|c|c|c|}
\hline MSSM-91 & $\mu$ & $M_{2}$ & $M_{1}$ & $M_{A}$ & $\tan \beta$ & $A_{t}$ & $A_{b}$ & $M_{\tilde{q}}$ & $M_{\tilde{\ell}}$ \\
MSSM-92 & $\mu$ & $M_{2}$ & $M_{1}$ & $M_{A}$ & $\tan \beta$ & $A_{t}$ & $A_{b}$ & $M_{\text {sf }, 1+2 \text { nd gen. }}$ & $M_{\text {sf }, 3 r d \text { gen. }}$ \\
MSSM-93 & $\mu$ & $M_{2}$ & $M_{1}$ & $M_{A}$ & $\tan \beta$ & $A_{t b}$ & $M_{\tilde{q}}$ & $M_{\tilde{\ell}, 1+2 \text { nd gen. }}$ & $M_{\tilde{\ell}, 3 \text { rd gen. }}$ \\
MSSM-94 & $\mu$ & $M_{2}$ & $M_{1}$ & $M_{A}$ & $\tan \beta$ & $A_{t b}$ & $M_{\tilde{t}_{R}}$ & $M_{\tilde{t}_{L} / \tilde{b}_{L}}$ & $M_{\text {sf, rest }}$ \\
\hline
\end{tabular}

Table 4. Free parameters for the four types of MSSM models considered in this work. Note that for the last two models we assume $A_{t}=A_{b}$.

As we will see, some of the most relevant part of the parameter space involves SUSY spectra with degenerate particle states, that are typically more difficult to test in proton collider experiments.

\subsection{Theoretical benchmark models}

We introduce four phenomenological "pMSSM-9" realisations, each defined by 9 parameters at the electroweak scale as specified below (see also table 4):

MSSM-91. The Higgsino mass parameter $\mu$, the Bino and Wino masses $M_{1}$ and $M_{2}$, the $C P$-odd Higgs boson mass $M_{A}$, the ratio of Higgs vacuum expectation values $\tan \beta$, the squark and slepton mass terms $M_{\tilde{\mathrm{q}}}$, and $M_{\tilde{\ell}}$, and the third generation trilinear couplings, $A_{t}$ and $A_{b}$ (note that $M_{1}$ and $M_{2}$ are not constrained by the GUT unification relation).

MSSM-92. Here, instead of distinguishing squark and slepton masses, we decouple the $3^{\text {rd }}$ sfermion generation from the $1^{\text {st }}$ and $2^{\text {nd }}$ unified generations.

MSSM-93. Squark and slepton mass terms are decoupled as in MSSM-91. Here, we allow for a separate $3^{\text {rd }}$ generation slepton mass, and a common slepton mass for the $1^{\text {st }}$ and $2^{\text {nd }}$ generation, respectively. In this case we assume $A_{t}=A_{b}$.

MSSM-94. Adding more freedom to the squark sector, we allow here for an independent right-handed stop (UR) mass and left-handed $3^{\text {rd }}$ generation squark mass (L), while adopting a universal sfermion mass for all other cases.

In addition, we used in all cases a fixed value of $M_{3}=5 \mathrm{TeV}$ for the gluino mass parameter. We performed Bayesian scans over the parameter space of these models by using MultiNest [63], which we interface to DarkSUSY to compute all relevant quantities that enter in the likelihood evaluations. The joint likelihood that we adopt takes the form

$$
\ln \mathcal{L}_{\text {Joint }}=\ln \mathcal{L}_{\Omega_{\chi} h^{2}}+\ln \mathcal{L}_{m_{\text {Higgs }}}+\ln \mathcal{L}_{\mathrm{SUSY}}+\ln \mathcal{L}_{\mathrm{LUX}}
$$

where $\mathcal{L}_{\Omega_{\chi} h^{2}}$ refers to the constraint on the cold DM relic abundance from cosmic microwave background $(\mathrm{CMB})$ observations; $\mathcal{L}_{m_{\mathrm{Higgs}}}$ imposes the mass of the lightest SUSY Higgs to agree with the Higgs boson mass; $\mathcal{L}_{\text {SUSY }}$ includes constraints from sparticles searches at colliders; and $\mathcal{L}_{\text {LUX }}$ accounts for the constraints on DM-nucleon interactions from the LUX direct detection experiment. 


\begin{tabular}{|lcl|}
\hline Model parameters & Low-mass & High-mass \\
\hline$\mu[\mathrm{GeV}]$ & $(70,2000)$ & $(500,4000)$ \\
$M_{1}[\mathrm{GeV}]$ & $(70,2000)$ & $(500,4000)$ \\
$M_{2}[\mathrm{GeV}]$ & $(70,2000)$ & $(500,4000)$ \\
$M_{\tilde{q}}, M_{\tilde{\ell}}\left(M_{\mathrm{sf}, i}\right)[\mathrm{GeV}]$ & $(70,4000)$ & $(500,4000)$ \\
$A_{t} / M_{\tilde{t}_{R}}, A_{b} / M_{\tilde{t}_{R}}$ & $(-3,3)$ & $(-3,3)$ \\
$M_{A}[\mathrm{GeV}]$ & $(70,2000)$ & $(500,4000)$ \\
$\tan \beta$ & $(5,40)$ & $(5,40)$ \\
\hline
\end{tabular}

Table 5. Parameter ranges of the scans performed. The range for $M_{\mathrm{sf}, i}$ applies to all combinations of independent sfermion mass parameters for the four models.

The relic abundance is computed including co-annihilations [64, 65], using a central value of $\Omega_{\chi} h^{2}=0.1198$ [1] and a generous error of $20 \%$ to account for both experimental and, more importantly, theoretical uncertainties in the $\Omega_{\chi} h^{2}$ prediction. We impose the predicted mass of the lightest Higgs to match the measured Higgs mass, $m_{h}=$ $125.09 \pm 0.24 \mathrm{GeV}$ [66]. Since we are mainly interested in suppression lifting mechanisms, rather than a detailed phenomenological analysis of the MSSM parameter space, we adopt a conservative set of further constraints to roughly indicate some of the more relevant experimental constraints. Apart from LEP and TeVatron constraints as implemented in DarkSUSY [39], we impose LHC constraints on stop, sbottom, light squark and slepton masses assuming direct production [67-77], as well as null results from direct DM detection [78].

For each model, we performed scans with two different parameter ranges: one for low-mass neutralinos, roughly $\sim 50-2000 \mathrm{GeV}$, and one for high masses, $\sim 500-3000 \mathrm{GeV}$, adopting logarithmic priors on the parameters, except for the trilinear couplings $A$. Table 5 contains the parameters and the ranges of the "low-mass" and "high-mass" scans. We emphasise that we do not perform global scans of the MSSM in light of the most recent results from various experiments (for this, see instead ref. [79]), nor is it our purpose to do so here. In particular, we note that important additional constraints can arise from electroweakino and MSSM Higgs searches, that are however highly dependent on the specific configuration of masses and decay channels, and therefore beyond the scope of this work. Rather than identifying the most probable parameter regions of our pMSSM-9 models, taking into account all experimental constraints, our focus is simply to provide a phenomenological proof of concept concerning the impact of three-body final states on the annihilation of DM particles and on the resulting cosmic-ray fluxes.

\subsection{Total annihilation rate}

In our extensive scans, we identified many MSSM models where the total annihilation cross section is significantly enhanced by including the three-body final states we consider here. As an illustration, we show in figure 6 the ratio between the (NWA subtracted) three-body and total two-body cross section, in function of the neutralino mass. In the left panel, we furthermore indicate the neutralino composition, while in the right panel we indicate the models where the neutralino is either close to a threshold or there exists an almost 

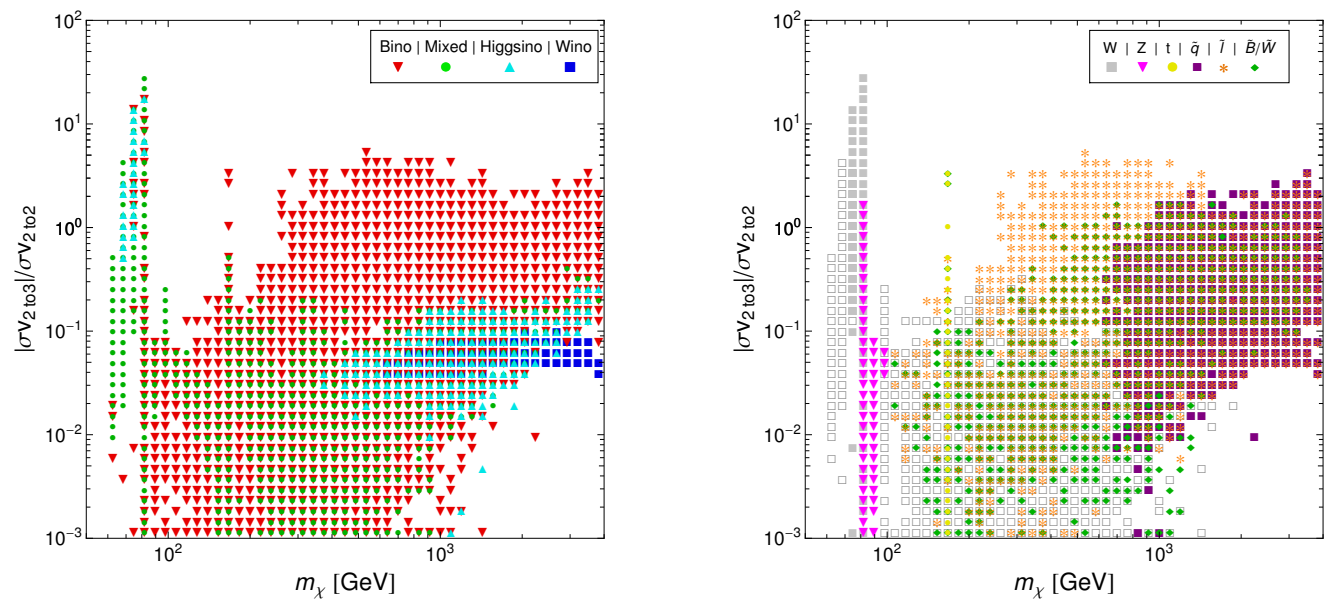

Figure 6. Left panel: ratio between the (NWA subtracted) three-body and total two-body cross section for the MSSM models defined in section 5.1, as a function of the lightest neutralino mass $m_{\chi}$. Different neutralino compositions are indicated as "Bino" $\left(\left|N_{11}\right|^{2}>0.8\right)$ in red, "Wino" $\left(\left|N_{12}\right|^{2}>0.8\right)$ in blue, "Higgsino" $\left(\left|N_{13}\right|^{2}+\left|N_{14}\right|^{2}>0.8\right)$ in cyan, and "mixed" (otherwise) in green. Right panel: same, but now broken down to models that are either (a) close to a threshold $X=W, Z, t$ (with $-4 \Gamma_{X}<m_{\chi}-m_{X}<\Gamma_{X}$, where $\Gamma_{X}$ is the total width of $X$ ) or (b) with a degenerate sfermion $\tilde{f}$ in the mass spectrum $\left(m_{\chi} / m_{\tilde{f}}>0.9\right)$ or $(c)$ Bino-like neutralinos with Wino coannihilation $\left(m_{\chi} / M_{2}>0.9\right.$ and $\left|N_{11}\right|^{2}>0.98$, without degenerate sfermions).

mass-degenerate SUSY particle. As anticipated, models with large enhancements indeed broadly fall into those two classes. In particular, cross section enhancements by $\mathcal{O}(10)$ factors are possible for models with neutralino masses close to the kinematical threshold of producing $W^{+} W^{-}, Z Z$ and $\bar{t} t$ final states; on the other hand, we did not find any models where the total cross section is significantly enhanced below the $Z h$ threshold. For models with neutralino masses close to the threshold for annihilation into a heavy Higgs boson and a SM particle $\left(Z H^{0}, A^{0} h, W^{ \pm} H^{\mp}\right)$ we identified up to $\sim 10 \%$ enhancements.

The second class of enhancement mechanisms are models with small mass splittings between the neutralino and other SUSY particles. For neutralino masses $m_{\chi} \gtrsim 200 \mathrm{GeV}$, degenerate sleptons can significantly increase $\sigma v$, while for even heavier neutralinos, $m_{\chi} \gtrsim$ $600 \mathrm{GeV}$, this also becomes possible for degenerate squarks. A final, somewhat less expected class of models where $\sigma v_{2 \rightarrow 3}$ can be of at least the same size as $\sigma v_{2 \rightarrow 2}$ are heavy Bino-like neutralinos with almost mass-degenerate Winos. Here, the relic density is set by Wino coannihilation, while the DM annihilation rate is highly suppressed due to either the small neutralino mixing (for $W W$ or $Z Z$ final states) or helicity factors $m_{f}^{2} / m_{\chi}^{2}$ (for fermionic final states). The lifting of the latter by three-body annihilation processes is thus very relevant in this case, even for models that do not feature degenerate sfermions.

One of the main new additions of this work is the full inclusion of final states containing a Higgs boson. To demonstrate their impact, we show in figure 7 the annihilation cross section to $H \bar{f} f / h \bar{f} f / A \bar{f} f / H^{ \pm} \bar{f} F$ as compared to the one for $Z \bar{f} f$ and $W^{ \pm} \bar{f} F$ final states. We find that Higgs boson IB alone can increase the lowest-order cross section by up to a factor of $\sim 3$, but in general the relative importance of the Higgs and gauge boson channels is very model dependent. Models where the former dominates (bottom right 


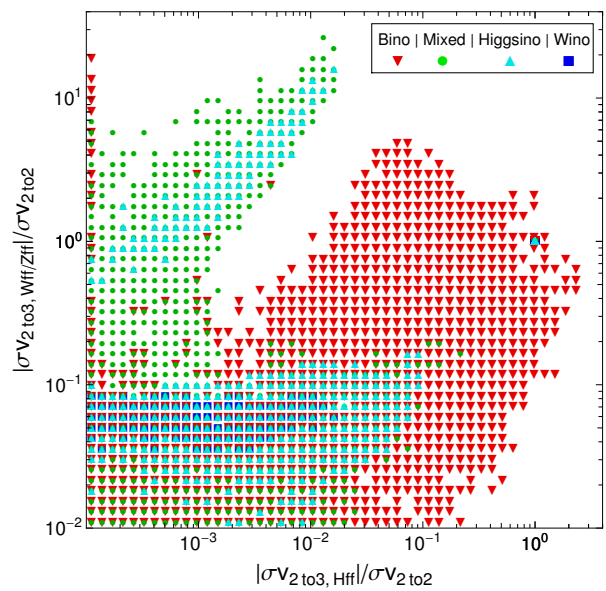

Figure 7. Cross section for annihilation into three-body final states containing a gauge boson versus that containing any of the Higgs bosons in the final state, normalized to the total two-body cross section. Different neutralino compositions are indicated as in figure 6 .

corner of the plot) feature neutralino masses in the $\mathrm{TeV}$ range, and a stop not much heavier than the neutralino (within a few hundred $\mathrm{GeV}$ ). The dominant annihilation channels are then $H t t, A t t, H^{ \pm} t b$, in particular via $\tilde{t}$ exchange in the $t / u$ channel, where the large Yukawa coupling to top quarks explains why the corresponding gauge boson final states are not equally pronounced. We note that these cases are examples where lifting of isospin suppression is more important than lifting of Yukawa suppression (see section 3.3).

A further class of models with large contributions of Higgs final states is characterized by large values of $\mu$ and $\tan \beta$, which lead to an enhancement of the Higgs coupling to sfermions. However, this enhances also the mixing between left- and right-handed sfermions and hence implies only a mild Yukawa suppression; nevertheless the three-body processes can be particularly important for the antiproton yield as discussed in more detail for benchmark model D3 further down.

For masses below the SM thresholds, annihilation into Higgs plus top final states is kinematically forbidden. For Binos, as clearly seen in the top left part of figure 7, this can result in large IB enhancements without Higgs contribution. For neutralinos with a significant Higgsino fraction, on the other hand, there is a correlation between gauge boson (mostly $W \bar{f} F$ ) and Higgs (mostly $h \bar{f} f$ ) final states (top middle part of figure 7 ). These models correspond to neutralino masses below the $W W$ threshold, and the three-body final states arise dominantly from virtual $W$ or $Z$ boson decays, $W W^{*} \rightarrow W \bar{f} F$ and $h Z^{*} \rightarrow h \bar{f} f$, respectively. The latter are suppressed compared to the former by the gaugino fraction, but otherwise of comparable size, which leads to the correlation ${ }^{10}$ seen in figure 7 .

\footnotetext{
${ }^{10}$ Note that this also explains the observed separation: the upper left part in the figure corresponds to threshold models, for which the Higgs contribution can be arbitrarily small in the pure Bino limit. The middle part corresponds to models where $t$-channel sfermion exchange is important. In this case, there is a $h \bar{f} f$ contribution even in the pure Bino limit, that arises from VIB emission of the Higgs boson, as discussed above. On the other hand, the gap between the pure Bino models on the very left part and the mixed and Higgsino-like models in the top middle part is due to (large, but still limited) statistics of our sample. The reason is that the ratio of $h \bar{f} f$ and $W / Z \bar{F} f$ cross section is extremely sensitive to the Higgsino fraction (roughly $\left.\propto\left(Z_{13}^{2}+Z_{14}^{2}\right)^{3}\right)$, and therefore varies very rapidly with the input parameters.
} 

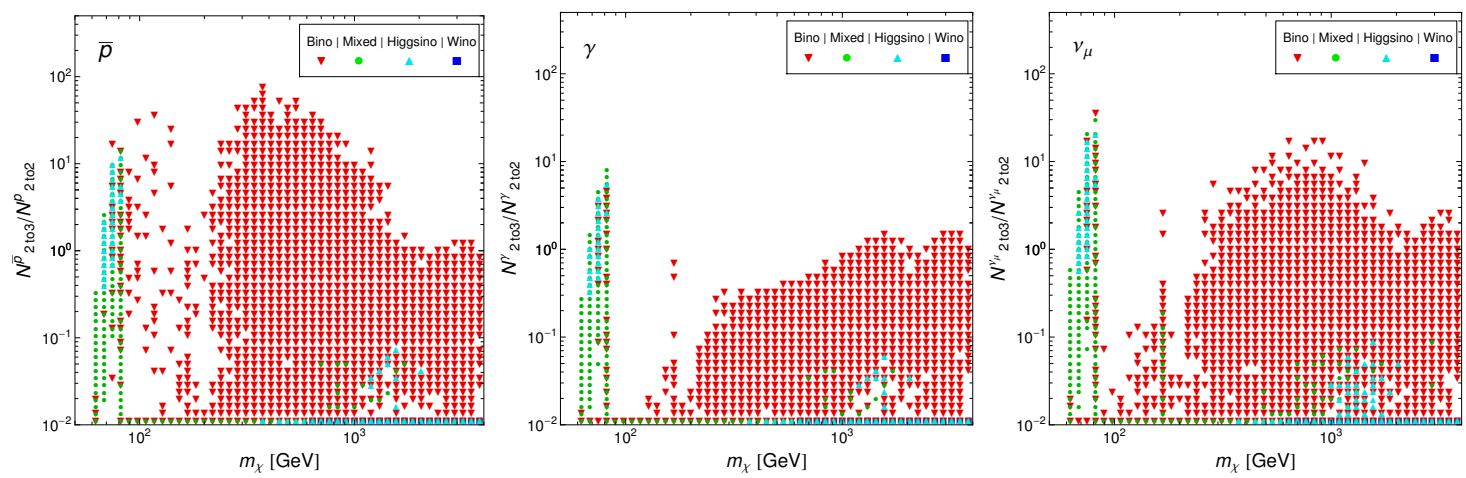

Figure 8. Ratio of integrated yields above $E_{X}>m_{\chi} / 10$ from (NWA-subtracted) three-body final states and the two-body result, for species $\mathrm{X}=\bar{p}, \gamma, \nu_{\mu}$, plotted against the neutralino mass $m_{\chi}$. The other light lepton yields $\left(\nu_{\tau}\right.$ and $\left.e^{+}\right)$are qualitatively very similar to the $\nu_{\mu}$ case. Different neutralino compositions are indicated as in figure 6 .
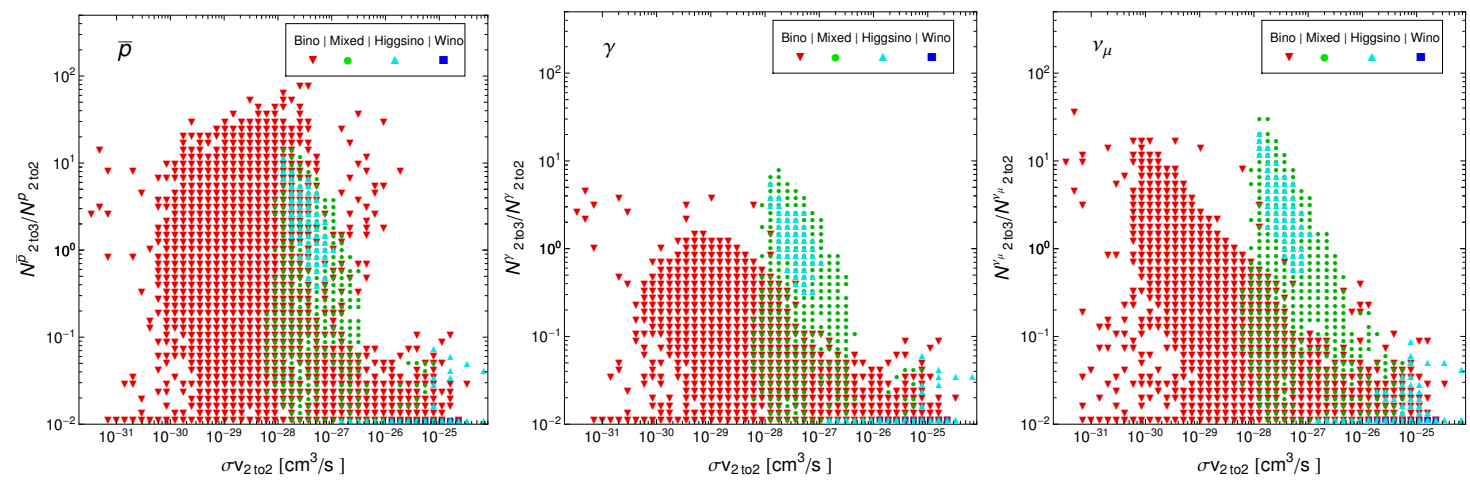

Figure 9. Same as figure 8, but now showing the integrated yields, for $E_{X}>m_{\chi} / 10$, against the total two-body cross section instead of the neutralino mass.

\section{$5.3 \quad$ Yield enhancement}

The zero-velocity annihilation cross section is already a good indicator for the reach of indirect detection experiments, but observationally more relevant is the resulting number of stable particles. Astrophysical background spectra are generally rather soft, i.e. they fall quickly with energy, such that from the point of view of indirect DM searches mostly annihilation products with relatively large energies are relevant (with the notable exception of CMB constraints that are mostly sensitive to the total energy deposition, see e.g. [80]).

In figures $8-10$, we therefore consider the integrated yield of all relevant stable particles $\mathrm{X}=\bar{p}, \gamma, \nu_{\mu}, \nu_{\tau}, e^{+}$above some threshold energy. Figure 8 , in particular, shows the ratio of the three-body yield to the typically considered yield expected from two-body final states, for $E_{X}>m_{\chi} / 10$, as a function of the neutralino mass and for different neutralino compositions. In figure 9 , we show the same quantity, but now as a function of the total two-body annihilation rate. This immediately allows us to make the interesting observation that the yields are most strongly enhanced for models with cross sections somewhat below the 'thermal' cross section of $\sim 10^{-26} \mathrm{~cm}^{3} \mathrm{~s}^{-1}$; including the effect of electroweak corrections, as 

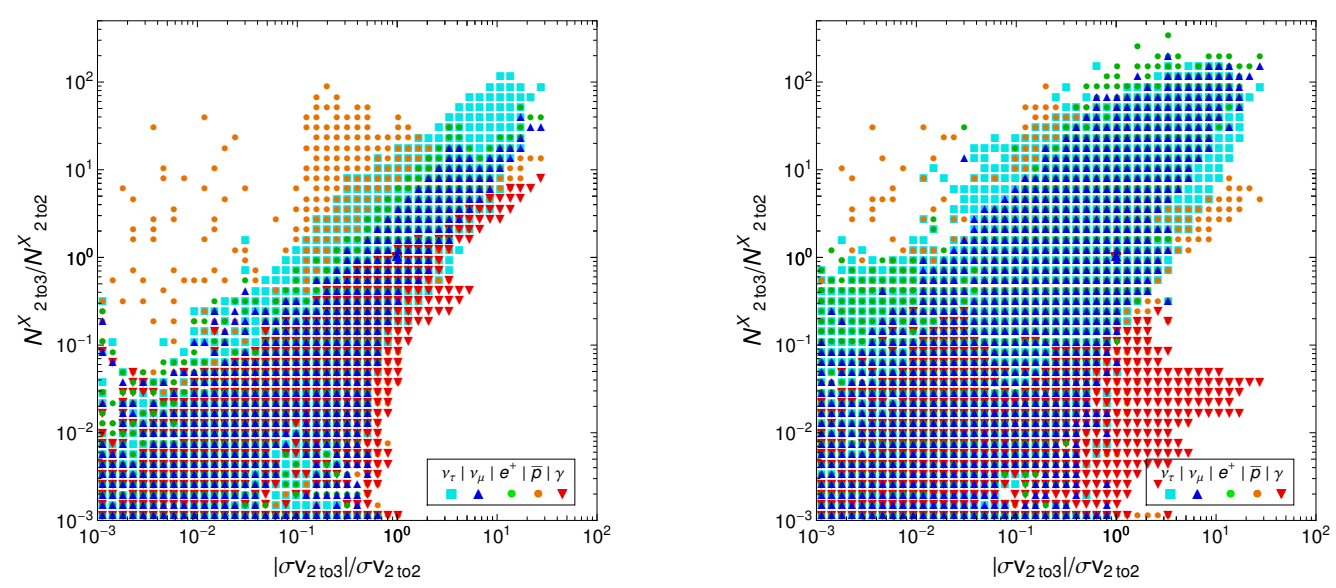

Figure 10. Same yield ratios as in figure 8, but now for all species $X=\bar{p}, \gamma, \nu_{\mu}, \nu_{\tau}, e^{+}$and plotted against the ratio of cross sections. Left panel: integrated yields above an energy threshold of $E_{X}>m_{\chi} / 10$. Right panel: integrated yields above an energy threshold of $E_{X}>m_{\chi} / 2$.

already pointed out in ref. [18], may thus turn out to be the decisive ingredient to make these models accessible by current and near-future indirect detection experiments. We complement these two sets of figures by figure 10, which demonstrates how the yield enhancement correlates with the cross section enhancement discussed in the previous subsection. This is done both for the yield enhancement at low energies (left) and at high energies close to the kinematic threshold (right), in order to get a first qualitative indication of how the spectral shape is affected. In the following, we discuss the various relevant stable particles in turn.

Antiprotons. For the bulk of the models considered here, the enhancement in the $\bar{p}$ yield scales as expected linearly with the enhancement in $\sigma v$. For $\tau^{+} \tau^{-}$final states, however, antiproton production is not kinematically allowed. Including the $\tau^{+} \tau^{-} Z$ and $\tau^{ \pm} \nu_{\tau} W^{\mp}$ channels can therefore drastically enhance the $\bar{p}$ yield even if the corresponding enhancement of the cross section is at most moderate. This effect, clearly seen in figure 10, is responsible for the largest enhancements (for Bino-like models) in the left panel of figure 8. It is also reflected in the left panel of figure 9, which shows that the enhancement is most pronounced for models with small two-body annihilation rates.

Antiprotons are only produced in the fragmentation and decay of the annihilation products, and therefore cannot obtain energies $E_{\bar{p}} \sim m_{\chi}$. The additionally emitted gauge or Higgs boson must first decay to quarks, inducing an additional step in the $\bar{p}$ production chain compared to two-body quark final states. The largest enhancement of the antiproton yield from three-body final states will thus on average occur mostly at small energies, an effect clearly seen when comparing the two panels of figure 10 .

Photons. Compared to antiprotons, gamma-ray yields lack the "atypical" enhancement from $\tau^{+} \tau^{-}$final states. Consequently, the enhancement in the yield is generally weaker, and more strongly correlated with the one in $\sigma v$. As for antiprotons, the spectrum is only enhanced at somewhat lower energies because the additional photons only result from steps further down in the decay chain. Unlike for the $\bar{p}$ case, on 
the other hand, our improved treatment of the NWA prescription for differential rates detailed in section 4.2 can actually decrease the photon yield at large energies (see section 5.4 for example spectra). The main reason for the large difference between the two panels of figure 10, however, are rather the monochromatic photon final states $\gamma Z$ and $\gamma \gamma$. While the annihilation rate into these states is loop-suppressed, they can still dominate the differential photon yield at the highest energies. ${ }^{11}$ The models where the photon-yield enhancement due to the inclusion of three-body final states is largest — which, from figure 8 are those close to the $W$ threshold - thus at the same time feature particularly large annihilation rates into $\gamma Z$ and $\gamma \gamma$, too.

Leptons. Unlike photons and antiprotons, leptons can appear directly in the final states considered here. This leads to a yield enhancement in particular at high energies, $E_{\ell} \gtrsim m_{\chi} / 2$, and with a strong correlation with the $\sigma v$ enhancement. Observationally, the resulting characteristic spectral features are especially relevant for positrons [10] in view of the excellent energy resolution of the AMS experiment [81], but also the neutrino spectra can be striking signatures to look for [82-84] if neutralino annihilation in the sun is sizeable (for more details, see the benchmark spectra discussed below).

As expected, $e^{+}$and $\nu_{e}$ yields are in general very similar, and the same holds for $\nu_{\mu}$. Large yield enhancements are in particular found (i) for models below the $W$ threshold, dominated by $\chi \chi \rightarrow W^{*} W \rightarrow \ell \nu W$, and (ii) for TeV models with almost degenerate sleptons and dominant three-body rates $Z \ell \ell$ and $W \ell \nu$. Compared to the other leptons, tau neutrinos can receive a somewhat larger yield enhancement, and generally feature a spectrum that is less pronounced at small energies. The reason for both effects is that the decay of the copiously produced pions, both from two-body final states and due to the additional final state boson, results in many low-energy $1^{\text {st }}$ and $2^{\text {nd }}$ generation leptons, but hardly any $3^{\text {rd }}$ generation leptons.

\subsection{Indirect detection spectra}

The additional final state boson may not only enhance the yields significantly, as described above, but also change the shape of the resulting cosmic-ray spectra in a characteristic way. The maybe most striking examples that we identified are sharp spectral features near the kinematic endpoint of neutrino and positron spectra, resembling in fact the often discussed cases of positron [14] and gamma-ray [13] spectra for photon VIB. In this section, we discuss in more detail a few example models where the spectrum of at least one type of stable particles changes significantly once three-body processes are taken into account.

For this purpose, we define seven pMSSM-9 benchmark models in table 6 , and collect in table 7 the phenomenological properties that are most relevant for our discussion. In particular, we include two threshold models, T1 and T2, with neutralino masses just below the

\footnotetext{
${ }^{11}$ Note that, throughout the manuscript, we include final states that vanish at tree-level when referring to two-body final states. This is not only the usual convention adopted in DarkSUSY, but serves to stress our emphasis on the differences between two-body and three-body final states (rather than between tree-level and next-to-leading order results).
} 


\begin{tabular}{|c|c|c|c|c|c|c|c|c|c|c|c|c|}
\hline $\begin{array}{c}\text { Bench } \\
\text { mark }\end{array}$ & Type & $\begin{array}{c}\mu \\
(\mathrm{GeV})\end{array}$ & $\begin{array}{c}M_{2} \\
(\mathrm{GeV})\end{array}$ & $\begin{array}{c}M_{1} \\
(\mathrm{TeV})\end{array}$ & $\begin{array}{c}M_{A} \\
(\mathrm{GeV})\end{array}$ & $\tan \beta$ & \multicolumn{2}{|c|}{$A\left(M_{\tilde{q}}\right)$} & \multicolumn{2}{|c|}{$M_{\tilde{q}}(\mathrm{GeV})$} & \multicolumn{2}{|c|}{$M_{\tilde{\ell}}(\mathrm{GeV})$} \\
\hline D1 & 93 & -2022 & 1257 & 1125 & 2841 & 12.4 & -2.00 & 2.00 & 1715 & 1715 & 1180 & 1147 \\
D2 & 94 & 3531 & 3458 & 3396 & 2562 & 22.5 & 1.94 & 1.94 & 3599 & $3665^{\mathrm{R}}$ & 3599 & 3599 \\
D3 & 93 & 3586 & 411.9 & 380.2 & 1766 & 11.7 & -1.43 & -1.43 & 3714 & 3714 & 485.3 & 470.1 \\
T1 & 91 & -102.0 & 601.7 & 119.8 & 521.1 & 12.5 & -2.00 & -1.81 & 1350 & 1350 & 1072 & 1072 \\
T2 & 91 & -586.4 & 189.0 & 172.6 & 981.4 & 8.66 & -1.56 & 2.34 & 1733 & 1733 & 698.7 & 698.7 \\
W & 93 & -3717 & 1346 & 1319 & 3160 & 9.01 & -1.91 & -1.91 & 2872 & 2872 & 1748 & 1885 \\
H & 93 & 3492 & 3976 & 3371 & 1418 & 9.47 & 1.57 & 1.57 & 3629 & 3629 & 3405 & 3783 \\
\hline
\end{tabular}

Table 6. Benchmark models for which we show the resulting spectra of stable particles in figures 1114, with model parameters for the various pMSSM-9 types introduced in section 5.1. $A_{t / b}$ are given in units of $M_{\tilde{t}_{R}}$. Model D2 takes as input the right-handed stop mass $(\mathrm{R})$, the left-handed third generation squarks mass (L), and otherwise assumes a common mass scale for all other sfermion mass terms. See table 7 for some phenomenological properties, and main text for more details.

\begin{tabular}{|c|c|c|c|c|c|c|c|c|c|}
\hline $\begin{array}{l}\text { Bench } \\
\text { mark }\end{array}$ & $\begin{array}{c}m_{\chi} \\
{[\mathrm{GeV}]}\end{array}$ & $\tilde{\chi}^{0}$ & $\begin{array}{c}m_{\tilde{q}}(\tilde{q}) \\
{[\mathrm{GeV}]}\end{array}$ & $\begin{array}{l}m_{\tilde{l}}(\tilde{l}) \\
{[\mathrm{GeV}]}\end{array}$ & $\begin{array}{c}m_{\tilde{\chi}^{i}} \\
{[\mathrm{GeV}]}\end{array}$ & $\frac{\sigma v_{3 b}}{\sigma v_{2 b}}$ & $\frac{\sigma v_{3 b, h}}{\sigma v_{3 b, W / Z}}$ & $\frac{N_{3 b, X}}{N_{2 b, X}}$ & $X$ \\
\hline D1 & 1125 & $\tilde{B}$ & $1551\left(\tilde{t}_{1}\right)$ & $1129\left(\tilde{\tau}_{1}\right)$ & 1254 & 0.58 & 0.12 & 61 & $\nu_{\mu}$ \\
\hline D2 & 3396 & $\tilde{B}$ & $3478\left(\tilde{b}_{1}\right)$ & $3397\left(\tilde{\tau}_{1}\right)$ & 3458 & 2.7 & 0.64 & 8.9 & $\nu_{\tau}$ \\
\hline D3 & 380.1 & $\tilde{B}$ & $3585.5\left(\tilde{t}_{1}\right)$ & $385.3\left(\tilde{\tau}_{1}\right)$ & 411.4 & 0.17 & 0.52 & 80 & $\bar{p}$ \\
\hline T1 & 80.34 & $\tilde{B} / \tilde{W}$ & $1176\left(\tilde{t}_{1}\right)$ & $1070\left(\tilde{\nu}_{e}\right)$ & 113.1 & 25 & $<10^{-3}$ & 43 & $e^{+}$ \\
\hline T2 & 172.4 & $\tilde{B}$ & $1604\left(\tilde{t}_{1}\right)$ & $693.7\left(\tilde{\tau}_{1}\right)$ & 188.8 & 3.0 & $<10^{-3}$ & 2.8 & $\nu_{\tau}$ \\
\hline W & 1319 & $\tilde{B}$ & $2720\left(\tilde{t}_{1}\right)$ & $1746\left(\tilde{\nu}_{e}\right)$ & 1345 & 0.39 & 0.12 & 26 & $\nu_{\mu}$ \\
\hline $\mathbf{H}$ & 3371 & $\tilde{B}$ & $3404\left(\tilde{b}_{1}\right)$ & $3400\left(\tilde{\mu}_{1}\right)$ & 3976 & 3.1 & 4.9 & 2.8 & $\nu_{\tau}$ \\
\hline
\end{tabular}

\begin{tabular}{|c|cc|cccc|}
\hline $\begin{array}{c}\text { Bench } \\
\text { mark }\end{array}$ & \multicolumn{2}{|c|}{$2 \rightarrow 2$} & & \multicolumn{3}{|c|}{$2 \rightarrow 3$} \\
\hline $\mathbf{D 1}$ & $g g(48 \%)$ & $b b(42 \%)$ & $W \nu \ell(37 \%)$ & $Z \ell \ell(33 \%)$ & $Z \nu \nu(9 \%)$ & $W t b(5 \%)$ \\
$\mathbf{D 2}$ & $H W / Z, h A(54 \%)$ & $g g(25 \%)$ & $W \nu \ell(30 \%)$ & $h \ell \ell(17 \%)$ & $Z \ell \ell(16 \%)$ & $H t b(10 \%)$ \\
$\mathbf{D 3}$ & $\tau \tau(99 \%)$ & $\gamma \gamma(0.3 \%)$ & $W \nu \ell(42 \%)$ & $h \ell \ell(37 \%)$ & $Z \ell \ell(20 \%)$ & \\
$\mathbf{T} 1$ & $c c(37 \%)$ & $g g(31 \%)$ & $W Q q(63 \%)$ & $W \nu \ell(33 \%)$ & $Z q q(2.6 \%)$ & \\
$\mathbf{T 2}$ & $W W(59 \%)$ & $g g(21 \%)$ & $W t b(99 \%)$ & & & \\
$\mathbf{W}$ & $g g(56 \%)$ & $b b(28 \%)$ & $W \nu \ell(31 \%)$ & $Z \ell \ell(28 \%)$ & $W t b(14 \%)$ & $Z \nu \nu(7.5 \%)$ \\
$\mathbf{H}$ & $H W / Z, h A(62 \%)$ & $g g(25 \%)$ & $H t b(42 \%)$ & $H t t(19 \%)$ & $A t t(17 \%)$ & $W t b(6.8 \%)$ \\
\hline
\end{tabular}

Table 7. Characteristic properties of the benchmark models defined in table 6 . The upper table shows neutralino mass and composition $(\tilde{B}=$ Bino-like, $\tilde{B} / \tilde{W}=\operatorname{mixed}$ Bino/Wino $)$, identity and mass of the lightest squark and slepton, next-to lightest neutralino, ratio of three-body to twobody cross section, ratio of three-body cross sections involving Higgs bosons to that involving weak gauge bosons, and the ratio of three-body to two-body yields for various species $X$ (integrated above $E_{X}>m_{\chi} / 10$ for D3, T1, T2 and above $E_{X}>m_{\chi} / 2$ for D1, D2, W, H). The lower table shows the dominant two- and three-body annihilation channels (for leptonic channels we sum over all three generations, while for quarks we quote separately the final states involving top quarks). 
$W$ and $t$ mass, respectively. Three of the benchmark models, D1 to D3, show a mass spectrum where at least one of the sfermions is degenerate in mass with the neutralino, while model $\mathrm{W}$ describes a TeV-scale Bino DM candidate where the correct relic density is obtained due to coannihilations with an almost degenerate Wino. Model H, finally, is a model example with a particularly large rate to three-body final states containing a Higgs boson.

Degenerate mass spectra. Models with all sleptons degenerate in mass with the neutralino show a significant overall enhancement of the yield in leptonic channels, $\ell=e^{ \pm}, \nu_{\mu}, \nu_{\tau}$, caused by sharp spectral features at the kinematic end-point of those spectra. In full analogy to the positron spectrum from VIB $e^{+} e^{-} \gamma$ final states [14], the annihilation in these models is dominated by $t$-channel diagrams with $\ell$ appearing directly in the final state (and additionally in the decay of the $W$ or $Z$ boson in the three-body final state); these diagrams lift the helicity suppression, and are maximized when the corresponding sleptons are degenerate with the neutralino. As an example of this type of models, we show in figure 11 the spectrum of benchmark D1. For leptonic final states (left panel) we can clearly see these sharp spectral features, leading to a yield enhancement as large as $\mathcal{O}(100)$ at high energies for all leptons. $\mathrm{SU}(2)$ corrections thus further enhance the $e^{+}$feature associated to photon IB, indicated separately with dotted lines, which the AMS experiment is highly sensitive to [10]. In addition, similar features appear also in neutrino final states, giving rise to a potential smoking-gun signature for annihilating Majorana DM at neutrino telescopes $[82,83,85] \cdot{ }^{12}$ In the right panel of figure 11 , we show instead the impact of radiative corrections on the gamma-ray and antiproton spectra. The impact of photon IB on the former is as expected large [13], while $\mathrm{SU}(2)$ corrections lead to much less significant, though still noticeable, spectral distortions. The antiproton spectrum only receives an overall enhancement directly related to the total $\sigma v$ enhancement.

If, on the other hand, only squarks are degenerate, then the lepton spectrum does not show any significant distortion but just an overall enhancement proportional to the one in $\sigma v$, while the photon spectrum again hardens slightly. In the left panel of figure 12 we show for illustration the case of benchmark model D2, which features both degenerate squarks and sleptons and hence, as expected qualitatively very similar spectra compared to those of D1. ${ }^{13}$

The spectra of benchmark model D3 (right panel of figure 12) are again qualitatively very different and feature a significant enhancement only in the antiproton channel. The reason, as already discussed in section 5.3, is that the two-body annihilation in D3 is largely dominated by $\tau \tau$ final states. Therefore, the three-body final states,

\footnotetext{
${ }^{12}$ If only first and second generation sleptons were degenerate in mass with the neutralino, a corresponding feature for $\nu_{\tau}$ (but not for $e^{ \pm}$and $\nu_{\mu}$ ) would be absent. The (non-)detection of such features can thus in addition be a powerful tool to robustly discriminate between such scenarios.

${ }^{13}$ One noticeable feature is the step-like behaviour of lepton yields from two-body annihilation (dashed lines). This drop by more than an order of magnitude, at $x \gtrsim 0.85$, is due the channels $H^{ \pm} W^{\mp}, h A$ and $H Z$ which contribute about $50 \%$ to the two-body cross section: the $W / Z$ decays constrain the resulting lepton energy to $E_{\ell}<E_{W / Z}=m_{\chi}\left(1-\left(m_{H}^{2}-m_{W / Z}^{2}\right) /\left(4 m_{\chi}^{2}\right)\right) \sim 0.85 m_{\chi}$ (for $m_{H}=2.6 \mathrm{TeV}$ as in D2). The threebody yields (solid lines) smear out the abrupt step (for $\nu_{\mu}$ ) or lead to a pronounced bump at $x \sim 1$ (for $\nu_{\tau}$ ).
} 

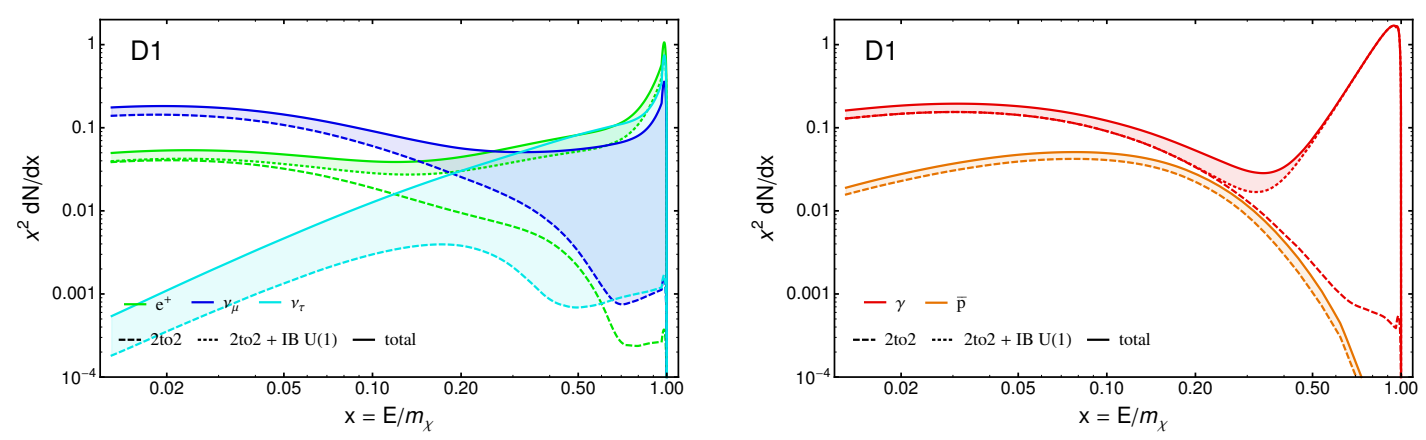

Figure 11. Spectral energy distribution of a model where all sleptons are degenerate (D1 in table 6). In the left panel, we display leptonic final states $e^{ \pm}, \nu_{\mu}$, and $\nu_{\tau}$ (green, blue and cyan line respectively) and, in the right panel photon (red) and antiproton (orange) spectra. Solid lines indicate the total (NWA-corrected two-body plus three-body) contribution, the dashed lines the twobody result. The dotted lines represent the contribution from two-body final states plus that from photon bremsstrahlung alone. Shaded areas thus highlight the effect of including $\mathrm{SU}(2)$ corrections.
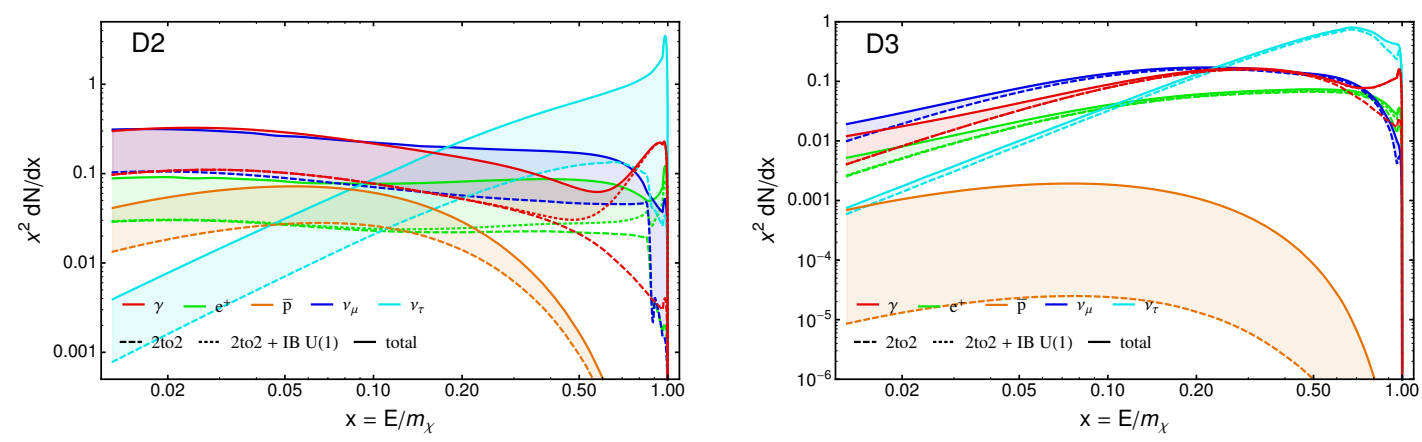

Figure 12. Same as figure 11, but now for models where both squarks and sleptons are degenerate in mass with the neutralino (model D2, left panel) or with a degenerate stau that is a mixture of $\tilde{\tau}_{L}$ and $\tilde{\tau}_{R}$ (model D3, right panel).

specifically $h \tau \tau$ and $W \tau \nu$, lead to a very large enhancement of the $\bar{p}$ flux - even though this only leads to a relatively small $(\sim 20 \%)$ correction in $\sigma v{ }^{14}$

Threshold models. In figure 13, we display the spectra for two models just below the $W$ and top quark threshold, corresponding to models T1 and T2 from table 6, respectively. Models with $m_{\chi} \sim m_{W}$ show a strong enhancement in all channels and at all energies. In particular, the three-body contributions induce pronounced bump-like features for $\nu_{\mu}, \nu_{\tau}, e^{ \pm}$spectra at $x \sim 0.7$, which result from the decay $W^{(*)} \rightarrow \ell \nu_{\ell}$. As expected from the discussion in section 3.5, these enhancements are most signifi-

\footnotetext{
${ }^{14}$ This model features a very large $\mu$-term $(\sim 3.5 \mathrm{TeV})$ and $\tan (\beta) \sim 12$, leading to a large stau mixing and hence only a mild helicity suppression for $\chi \chi \rightarrow \tau^{+} \tau^{-}$. Corrections to leptonic channels are thus small in this specific case, despite an almost degenerate stau. We also note that $h \tau \tau$ final states, via Higgs VIB, are enhanced with respect to gauge boson IB in this type of model. The reason is that the mixing contribution $\propto g \mu v_{E W} \tilde{\tau}_{L} \tilde{\tau}_{R}$ to the stau mass is directly linked to a large $\tilde{\tau}_{L} \tilde{\tau}_{R} h$ coupling to the Higgs boson (since the VEV and the Higgs field appear in the combination $v_{E W}+h$ in the Lagrangian).
} 

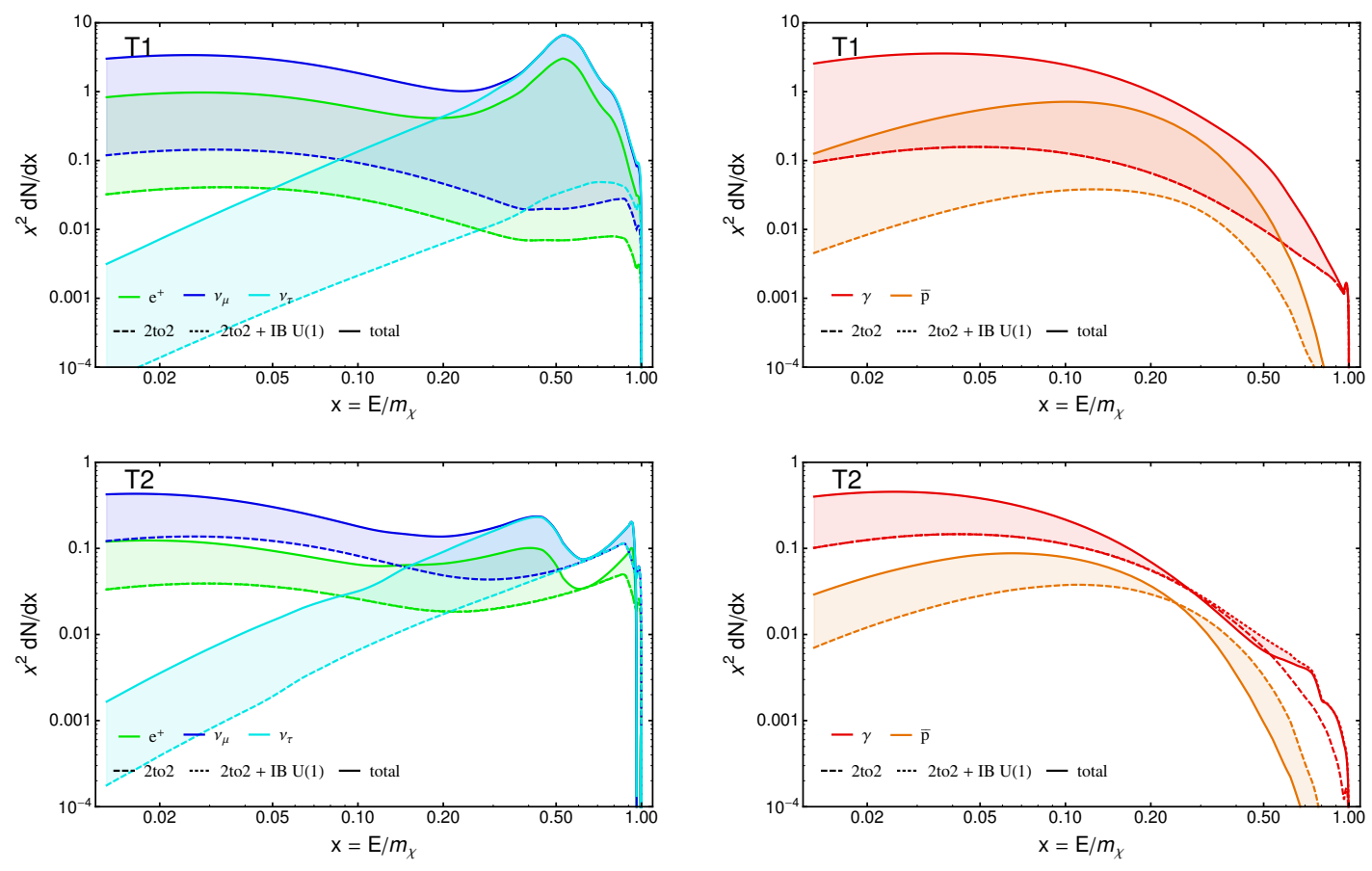

Figure 13. Same as figure 11, but for a $W$ threshold model (T1, top) and a top-quark threshold model (T2, bottom). The features in the shape of the lepton and gamma ray spectra are due to an interplay of various effects as discussed in detail in the text.

cant for $m_{\chi}$ slightly below $m_{W}$. Photon and antiproton spectra, on the other hand, are somewhat softer than the two-body spectra, but can be greatly enhanced at all energies.

We also find enhancements in all channels when $m_{\chi} \sim m_{t}$, and observe a peculiar double structure in the spectrum of leptonic channels for T2: a bump-like feature at $x \sim 0.4$ and a sharp spectral feature at higher energies. The first is directly related to the dominant off-shell top decay close to the threshold, $\chi \chi \rightarrow t \bar{t}^{(*)} \rightarrow W t b$. The line-like feature close to $x=1$, on the other hand, arises from leptonic decays of the transversely polarized $W$-bosons in the process $\chi \chi \rightarrow W \bar{W} \rightarrow W \ell \nu_{\ell}{ }^{15}$

For the antiproton and gamma spectra, finally, the inclusion of the three-body result causes relatively large deviations as the two-body yields can both increase and decrease, depending on the energy. ${ }^{16}$

\footnotetext{
${ }^{15}$ Note that the NWA-subtracted cross section for $W \ell \nu_{\ell}$ is much smaller than for the kinematically accessible $\chi \chi \rightarrow W^{+} W^{-}$. The enhancement at $x \sim 1$ originates thus exclusively from our three-body computation taking the (transverse) $W$ polarization into account (see appendix C, specifically eq. (C.37)). This type of correction can occur whenever $m_{\chi} \gg m_{W}\left(m_{Z}\right)$ and $\chi \chi \rightarrow W^{+} W^{-}, Z Z$ proceeds with a significant rate.

${ }^{16}$ The peculiar shape of the gamma-ray spectrum, in particular, can be explained as follows: for low energies $x \lesssim 0.2$ there is a strong enhancement from $W t b$ final states, while for intermediated energies $x \sim 0.2-0.6$, the spectrum is slightly suppressed compared to the two-body case. At high energies $x \gtrsim 0.6$, $W W \gamma$ and $\bar{\ell} \ell \gamma$, i.e. photon IB final states dominate; the sharp drop around $x \sim 0.8$ is due to the kinematic endpoint of the $W W \gamma$ contribution, while photons from $\bar{\ell} \ell \gamma$ dominate for $x>0.8$.
} 

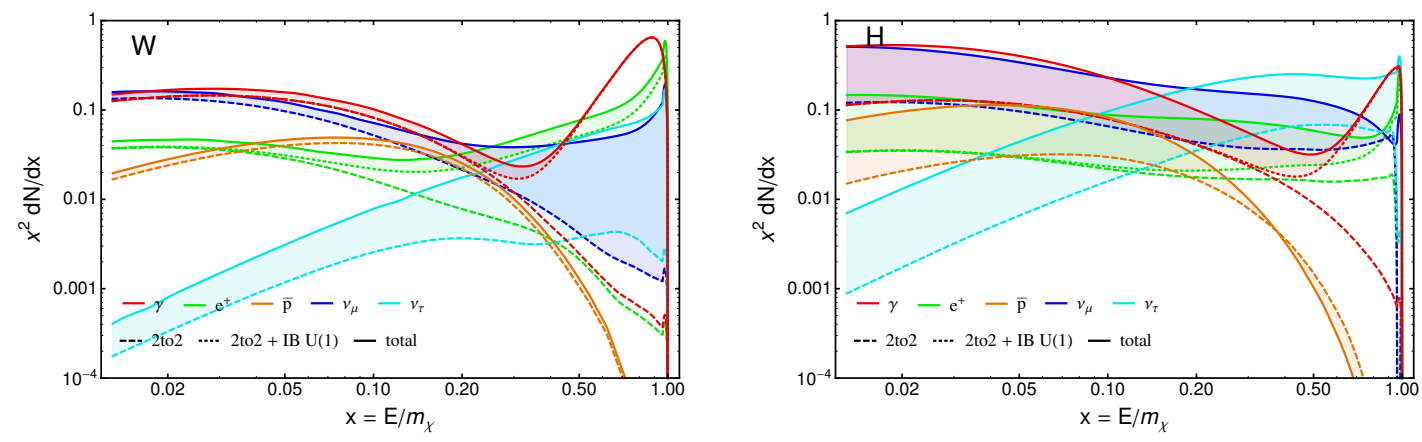

Figure 14. Same as figure 11, but for a Bino-like neutralino with almost degenerate Wino (W, left) and a model with large $H f \bar{f}$ contribution (H, right). The features in the shape of the lepton and gamma ray spectra are due to an interplay of various effects as discussed in detail in the text. Left panel: bino-like neutralino with almost degenerate Wino (benchmark model W). Final state channels: photons (red), antiprotons (orange), positrons (green), $\nu_{\mu}$ (blue), and $\nu_{\tau}$ (cyan). Solid lines indicate the total (two-body and three-body) contribution, the dashed lines the two-body process. Right panel: large $H f \bar{f}$ contribution (benchmark model $\mathrm{H}$ ).

Special cases. In figure 14, we show two interesting cases that do not fall in either of the categories above. In the left panel, we present benchmark model W, a Binolike neutralino degenerate with the Wino. The (small) two-body annihilation rate is dominated by $g g$ final states, followed by $\bar{f} f$. The three-body process thus lifts the helicity suppression of the latter and can be important even if the sfermions are not highly degenerate in mass with the neutralino. Because the contribution to the neutrino and positron spectra still come dominantly from $W \nu \ell$ final states, they show sharp spectral features like in models with even more degenerate sleptons. The right panel of figure 14, instead, corresponds to a model with a large $(\sim 85 \%)$ contribution to the cross section from channels that involve the MSSM Higgs bosons and top quarks (benchmark model $\mathrm{H})$. The neutralino mass is rather heavy $(\sim 3.3 \mathrm{TeV})$ such that even $t \bar{t}$ final states suffer from a certain amount of helicity suppression. Due to the large top Yukawa coupling, the suppression is lifted preferably via Higgsstrahlung. For this model, leptons are dominantly produced indirectly, and correspondingly lepton spectra are enhanced broadly at all energies. The small additional spike at very high energies results from the $W / Z$ decay from $W \bar{F} f(10 \%)$ and $Z \bar{f} f(5 \%)$ final states.

In figure 15, finally, we show for a subset of our benchmark models the ratios of threebody to two-body yields, illustrating some of the features discussed above on a model-bymodel basis from a slightly different angle. We note in particular the strong enhancement of high-energy lepton spectra for model $\mathrm{H}$, which is explained - similar to the situation for model D2 - by a sharp drop in the two-body yield from $W^{ \pm} H^{\mp}$ and $Z H$ due to the maximal lepton energy from $W / Z$ decays that is kinematically possible.

A widely used phenomenological approach to take into account electroweak corrections to DM annihilation spectra, often referred to as 'model-independent' in the literature, is based on splitting functions inspired by a parton picture [16, 17]. These effectively 

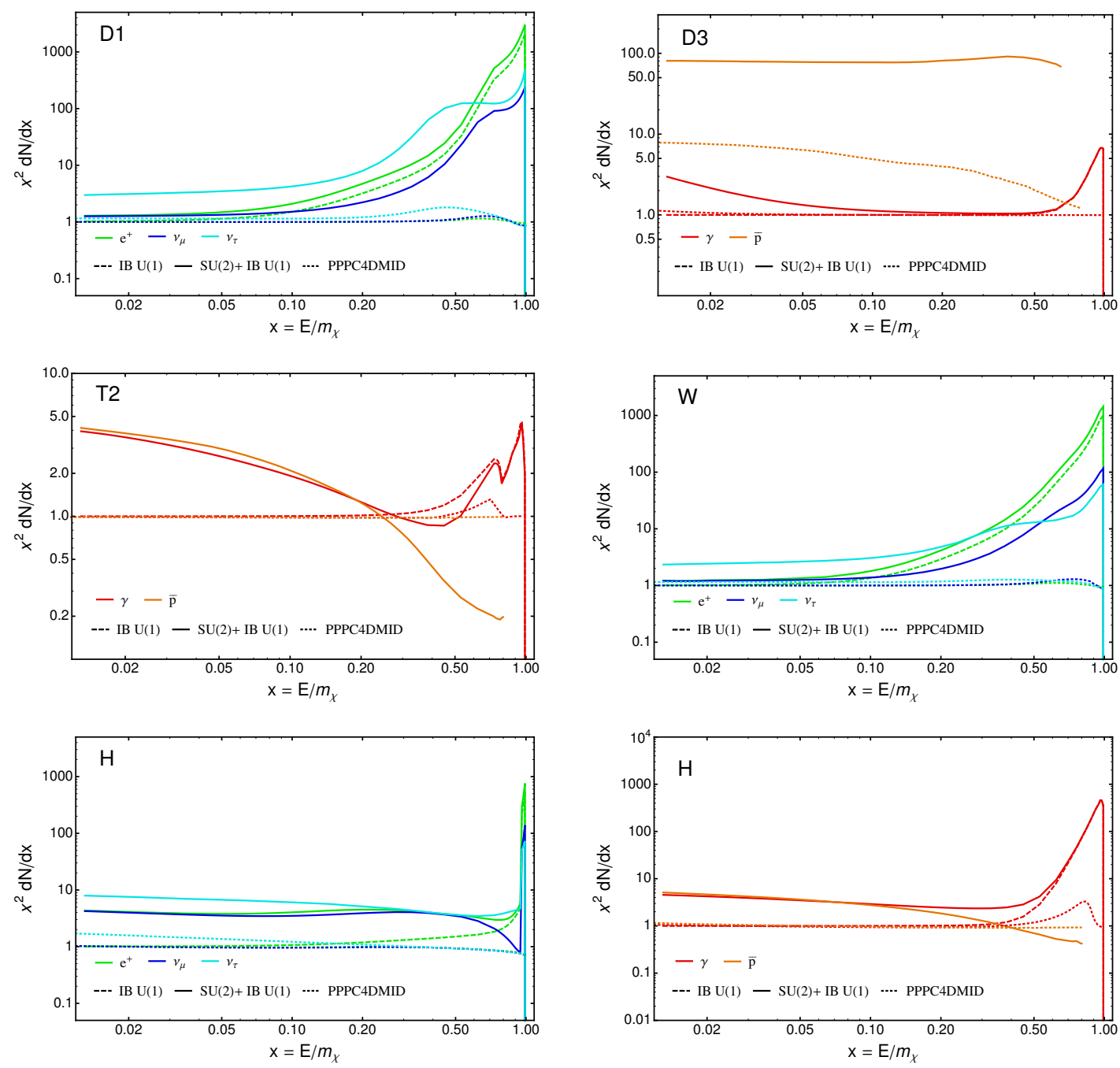

Figure 15. Ratio of three-body to two-body yields, for some of the benchmark models from table 6 . Solid lines show the full ratios, while dashed lines indicate the contribution from photon IB alone. Dotted lines show the result from the method implemented in the 'Poor Particle Physicist Cookbook for Dark Matter Indirect Detection' (PPPC4DMID) [17]; note that the relatively small resulting corrections are not restricted to these benchmark models but generic for neutralino annihilation. We cut the $\bar{p}$ spectrum at $x \sim 0.8$ in order to avoid numerical artefacts from very small, and hence poorly sampled, two-body yields provided by DarkSUSY. See text for more details.

result from assuming point-like interactions being responsible for the two-body annihilation channels, such that three-body final states are dominated by gauge (or Higgs) bosons that are soft or collinear with the final state particle they are radiated from. In figure 15, we therefore also indicate the ratios that result from this approach. ${ }^{17}$ As one can see, the resulting changes in the yield differ at times drastically. In fact, for the pMSSM models

\footnotetext{
${ }^{17}$ Since only the SM Higgs boson is implemented in [17], we have approximated the missing yields by replacing the heavy MSSM Higgs bosons with Goldstone bosons, i.e. longitudinal gauge boson components. For the sake of figure 15 we thus implemented, e.g., $h A$ final states as $50 \%$ hh and $50 \% Z_{L} Z_{L}$ final states.
} 
studied here, we find much more generally that the full annihilation spectra from final states containing fermions deviate substantially from the model-independent approximation whenever electroweak corrections induce even $\mathcal{O}(10 \%)$ changes to the two-body rates. Also in the case of $\mathrm{TeV} \mathrm{DM}$ models, the difference between two-body and corrected yields is much larger than what one would expect when adopting the PPPC [17] implementation.

For neutralino annihilation, this is quite straight-forward to understand, and for this reason we also expect these conclusions to hold in even more generic MSSM models. In the 'model-independent' prescription, in particular, none of the enhancement mechanisms described in detail in section 3 is captured. Final states containing leptons are thus necessarily still suppressed by $m_{\ell}^{2} / m_{\chi}^{2}$, while quark and gluon final states are hardly affected by electroweak corrections due to soft or collinear radiation [16] given the strong dynamics leading to essentially immediate fragmentation. Furthermore, MSSM specific final states (heavy Higgs bosons) are not included, and even for the final states that are included the energy distribution of the final state particles can differ substantially when taking into account the full matrix element or just assuming a point-like interaction, respectively. The latter is for example visible in the antiproton spectrum of model D3: as the two-body annihilation is dominated by $\tau$ lepton final states, the main contribution must come from the final state boson; in the PPPC case the softer antiproton spectrum can then be traced back to the fact that the emitted boson has a much softer spectrum.

\section{Conclusions}

In this article, we have studied in detail the annihilation of Majorana DM particles into a pair of fermions and an electroweak gauge or Higgs boson in the final state. We have revisited the arguments why the annihilation to fermionic two-body final states is helicity suppressed, and pointed out that this can be traced back to a combination of two fundamentally distinct effects, dubbed Yukawa- and isospin suppression, which can independently be lifted if the final state boson carries isospin. Furthermore, we have consistently generalized a standard way of avoiding 'double-counting' of contributions from two- and three-body final states, which consists in subtracting the latter in the narrow-width approximation, to differential cross sections and the yield of stable particles relevant for indirect DM searches. The latter constitutes one of our main results, which we believe will prove useful also in other contexts.

As a concrete application, we have performed the first full analytical calculation of all differential cross sections for the internal bremsstrahlung of electroweak gauge bosons, as well as any MSSM Higgs boson, for fermion final states from neutralino annihilation. We have performed a detailed analysis of these results in light of our general discussion, recovering specific examples pointed out previously in the literature, and extending them, but also pointing out qualitatively new processes. In order to estimate the size of the corrections reported here, we have performed dedicated scans over the parameter space of various MSSM realizations.

We find that both lifting of the Yukawa and lifting of the isospin suppression can significantly increase the total neutralino annihilation rate. Even more importantly, how- 
ever, the resulting spectra of positrons, neutrinos, antiprotons and gamma rays can differ substantially from those obtained for two-body final states - even when including the 'model-independent' electroweak corrections implemented in PPPC [17]. We stress that this is a generic result which is not restricted to specific cases but holds whenever electroweak corrections to fermionic channels are (at least) comparable to the two-body results, e.g. for TeV DM models. Given that the supersymmetric neutralino still is the prototype WIMP DM candidate, our results thus underline the importance of performing full computations consistent with the model that is being studied. In other words, such radiative corrections are intrinsically highly model-dependent, and even the often adopted 'modelindependent' approach of ref. [17] can be argued to simply rest on one rather specific model realization (in the sense that the underlying assumptions essentially describe a point like interaction, which is a good approximation under roughly the same conditions under which an effective operator analysis is valid to leading order in perturbation theory. ${ }^{18}$ )

To conclude, we have shown that the way electroweak corrections to DM annihilation are commonly estimated can lead to rather misleading results for a given DM model. The consistently computed spectra of stable particles from DM annihilation can be much larger or offer striking spectral features, either of which may significantly help to indirectly detect DM in forthcoming experiments. We stress that this holds for all yields relevant for indirect DM detection, i.e. both gamma rays, charged cosmic rays $\left(\bar{p}\right.$ and $\left.e^{+}\right)$and neutrinos. The routines needed to compute all relevant rates and particle yields for neutralino annihilation in the MSSM will be included in the shortly upcoming public release 6.0 of the DarkSUSY package [40].

\section{Acknowledgments}

We thank Maria Eugenia Cabrera Catalan, Marco Cirelli, Feng Luo, Are Raklev, Roberto Ruiz de Austri Bazan and Filippo Sala for useful discussions.

\section{A Neutralino annihilation amplitudes}

In this appendix, we review our analytical approach of calculating the matrix elements by means of an expansion in helicity amplitudes (A.1). For illustration of our full results, we then consider a number of phenomenologically interesting limiting cases concerning the composition of the lightest neutralino (A.2).

\section{A.1 Expansion of amplitudes in the helicity basis}

For the analytical calculation of amplitudes we closely follow the procedure of ref. [18], presented in detail in chapter 4 and corresponding appendices of ref. [87]. We thus modify the generic MSSM model file shipped with the FeynArts mathematica package [88] such

\footnotetext{
${ }^{18}$ Note that while some of the effects of helicity suppression lifting could in principle be described via additional higher-dimensional effective operators in specific regions of parameter space [86], it turns out that within the MSSM the part of parameter space in which this is possible typically does not coincide with the one where the corrections are most relevant.
} 
as to agree with the conventions adopted in DarkSUSY $[40,45]$. We then use FeynArts to generate all possible Feynman diagrams for neutralino annihilation into three-body final states containing a fermion, an anti-fermion and a Boson. In the next step, given that we want to restrict ourselves to the $v \rightarrow 0$ limit, we project out the singlet state $\left(J^{P}=0^{-}\right)$of the annihilating neutralino pair with total momentum $p$ by replacing the two external Majorana spinors in the amplitude with $P_{S_{0}} \equiv \frac{\gamma^{5}}{\sqrt{2}}\left(m_{\chi}-\not p / 2\right)$. Finally, we expand the amplitude for each diagram in terms of helicity amplitudes, applying a method used originally for neutralino annihilation to two-body final states [89] and extended to three-body final states in $[18,87]$.

Let us review those final steps in a bit more detail. By applying the $P_{1} S_{0}$ projector, in particular, we can reduce any of the matrix elements considered here to the generic form

$$
\mathcal{M} \propto \bar{u}_{r}\left(k_{1}\right) \Gamma_{r s} v_{s}\left(k_{2}\right) \epsilon_{\mu}^{*}\left(k_{3}\right)
$$

where only the final state spinors appear and $\Gamma$ is a $4 \times 4$ matrix. We use Feyncalc [90] to decompose $\Gamma$ into the standard basis of matrices where the corresponding Dirac bilinears are real and have definite transformation properties under the Lorentz group (i.e. scalar, vector, tensor, pseudo-vector and pseudo-scalar, respectively). In order to assign helicities, we work in the back-to-back frame of the outgoing fermion-antifermion pair, which we define by $\mathbf{k}_{\mathbf{1}}=-\mathbf{k}_{\mathbf{2}}$. For states of definite helicity $h= \pm 1 / 2$, this implies that we can use [91]

$$
\chi_{h}\left(\hat{\mathbf{k}}_{1,2}\right)=\chi_{-h}\left(-\hat{\mathbf{k}}_{1,2}\right)
$$

for the two-component spinors that appear in the explicit Dirac representations of both $u$ and $v$. Choosing the fermion momentum $\mathbf{k}_{1}$ to be aligned with the $z$-axis, we thus obtain

$$
u_{+}=\left(\begin{array}{c}
\eta_{k_{1}}^{+} \\
0 \\
\eta_{k_{1}}^{-} \\
0
\end{array}\right), \quad u_{-}=\left(\begin{array}{c}
0 \\
\eta_{k_{1}}^{+} \\
0 \\
-\eta_{k_{1}}^{-}
\end{array}\right), \quad v_{+}=\left(\begin{array}{c}
\eta_{k_{2}}^{-} \\
0 \\
-\eta_{k_{2}}^{+} \\
0
\end{array}\right), \quad v_{-}=\left(\begin{array}{c}
0 \\
\eta_{k_{2}}^{-} \\
0 \\
\eta_{k_{2}}^{+}
\end{array}\right) \text {, }
$$

where we have introduced $\eta_{k_{1,2}}^{ \pm} \equiv\left(k_{1,2}^{0} \pm m_{1,2}\right)^{1 / 2}$. In this frame, we can furthermore choose the momentum of the final state boson, $\mathbf{k}_{3}$, to lie in the $y-z$ plane, spanning an angle $\theta$ with the $z$-axis. For the case of a massive vector boson, the 3 possible polarization states $\epsilon_{\lambda}$ of definite helicity are thus given by

$$
\epsilon_{ \pm}=\frac{1}{\sqrt{2}}(0, \mp 1,-i \cos \theta, i \sin \theta), \quad \epsilon_{0}=\frac{1}{m_{3}}\left(\left|\mathbf{k}_{3}\right|, 0, k_{3}^{0} \sin \theta, k_{3}^{0} \cos \theta\right) .
$$

Singlet and triplet spin states of the two-fermion system can now be constructed from the individual helicity states as in eqs. (2.4), (2.5), i.e. we can decompose each bilinear as

$$
\begin{aligned}
(\bar{u} \Gamma v)_{(0,0)} & =\left(\bar{u}_{+} \Gamma v_{+}-\bar{u}_{-} \Gamma v_{-}\right) / \sqrt{2}, \\
(\bar{u} \Gamma v)_{(1,-1)} & =\bar{u}_{-} \Gamma v_{+}, \\
(\bar{u} \Gamma v)_{(1,0)} & =\left(\bar{u}_{+} \Gamma v_{+}+\bar{u}_{-} \Gamma v_{-}\right) / \sqrt{2}, \\
(\bar{u} \Gamma v)_{(1,+1)} & =\bar{u}_{+} \Gamma v_{-} .
\end{aligned}
$$




\begin{tabular}{|c|c|c|c|c|}
\hline$(\bar{u} \Gamma v)_{h}$ & $h=(0,0)$ & $h=(1,-1)$ & $h=(1,0)$ & $h=(1,1)$ \\
\hline $\bar{u} v$ & 0 & 0 & $p_{+}$ & 0 \\
$\bar{u} \gamma^{\mu} v$ & 0 & $E_{+} e_{+}^{\mu}$ & $p_{-} e_{u}^{\mu}-E_{-} e_{0}^{\mu}$ & $E_{+} e_{-}^{\mu}$ \\
$\bar{u} \sigma^{\mu \nu} v$ & $i p_{+}\left(e_{-}^{\mu} e_{+}^{\nu}-e_{+}^{\mu} e_{-}^{\nu}\right)$ & $i p_{-}\left(e_{+}^{\mu} e_{0}^{\nu}-e_{0}^{\mu} e_{+}^{\nu}\right)$ & $i E_{+}\left(e_{0}^{\mu} e_{u}^{\nu}-e_{u}^{\mu} e_{0}^{\nu}\right)$ & $i p_{-}\left(e_{-}^{\mu} e_{0}^{\nu}-e_{0}^{\mu} e_{-}^{\nu}\right)$ \\
& $+i E_{-}\left(e_{u}^{\mu} e_{+}^{\nu}-e_{+}^{\mu} e_{u}^{\nu}\right)$ & & \\
$\bar{u} \gamma^{\mu} \gamma^{5} v$ & $\left.p_{-} e_{u}^{\mu} e_{-}^{\nu}-e_{-}^{\mu} e_{u}^{\nu}\right)$ \\
$\bar{u} \gamma^{5} v$ & $-E_{+}^{\mu}$ & $-p_{+} e_{+}^{\mu}$ & 0 & $p_{+} e_{-}^{\mu}$ \\
\hline
\end{tabular}

Table 8. Decomposition of basis Dirac bilinears into helicity eigenstates of the two final state fermions. For a definition of the quantities appearing here, see eqs. (A.9)-(A.11).

In table 8, we show the result of this decomposition for each of the 16 basis Dirac bilinears, where for ease of notation we have introduced the following kinematic quantities (note the different normalization convention with respect to [87]):

$$
\begin{aligned}
& E_{ \pm} \equiv \sqrt{2}\left(\eta_{k_{1}}^{+} \eta_{k_{2}}^{+} \pm \eta_{k_{1}}^{-} \eta_{k_{2}}^{-}\right) \\
& p_{ \pm} \equiv \sqrt{2}\left(\eta_{k_{1}}^{+} \eta_{k_{2}}^{-} \pm \eta_{k_{1}}^{-} \eta_{k_{2}}^{+}\right)
\end{aligned}
$$

Four-vectors and tensors, furthermore, are more conveniently expressed in the helicity basis,

$$
\left\{\tilde{e}_{(\mu)}\right\}=\left\{\tilde{e}_{u}, \tilde{e}_{+}, \tilde{e}_{-}, \tilde{e}_{0}\right\} \equiv\left\{(1,0,0,0),\left(0, \frac{-1}{\sqrt{2}}, \frac{-i}{\sqrt{2}}, 0\right),\left(0, \frac{1}{\sqrt{2}}, \frac{-i}{\sqrt{2}}, 0\right),(0,0,0,1)\right\},
$$

which is an orthonormal basis choice just like the canonical coordinate basis $\left\{e_{(\mu)}\right\}$ with $\left(e_{(\mu)}\right)^{\nu}=\delta_{\mu}^{\nu}$. This implies, e.g., that the components of a four-vector $V$ for these basis choices are related by $V^{\mu}=A^{\mu}{ }_{\nu} \tilde{V}^{\nu}$, where $A_{\mu \nu} \equiv e_{(\mu)} \cdot \tilde{e}_{(\nu)}$.

With the above decompositions of fermion and vector boson polarizations even the full analytic expressions for the amplitudes turn out to be relatively easily manageable. We evaluate the amplitude for every helicity configuration, for each diagram separately, and simplify it further by explicitly contracting the remaining polarisation vectors (when applicable), basis vectors and four-momenta. We then sum over all diagrams to obtain the total helicity amplitudes $\mathcal{M}^{(h, \lambda)}$, where $h$ is the helicity of the fermion-antifermion pair in the back-to-back system and $\lambda$ the polarisation state of the emitted vector boson. Finally, we obtain the total amplitude squared by averaging over initial $(r, s)$ spins and summing over final $\left(r^{\prime}, s^{\prime}\right)$ degrees of freedom:

$$
\overline{\mid \mathcal{M}}^{2} \equiv \frac{1}{4} \sum_{r, s, r^{\prime}, s^{\prime}, \lambda}\left|\mathcal{M}_{\chi \chi \rightarrow \bar{F} f X}\right|^{2} \equiv \frac{1}{4} \sum_{h, \lambda}\left|\mathcal{M}_{\chi \chi \rightarrow \bar{F} f X}^{(h, \lambda)}\right|^{2},
$$

where $X$ is either a vector boson $(W / Z)$ or a scalar Higgs (in which case no polarisation is present). For convenience, we then transform back to the CMS frame. This allows us to compute the total three-body cross section by integrating over the phase space,

$$
\frac{d\left(\sigma v_{2 \rightarrow 3}\right)}{d E_{1} d E_{2}}=\frac{1}{16 m_{\chi}^{2}} \frac{1}{(2 \pi)^{3}}|\mathcal{M}|^{2}
$$


where $E_{1}$ and $E_{2}$ are the CMS energies of any two final state particles. For details concerning the numerical implementation, we refer to appendix B.

\section{A.2 Results for expanded amplitudes}

Let us consider our analytical results in the limit of heavy neutralino masses, which amounts to taking the ratio of the electroweak VEV and the neutralino mass, $\delta_{v} \equiv v_{E W} / m_{\chi}$, and expanding the full results for the amplitude around $\delta_{v}=0$. Besides allowing for compact analytic expressions, this limit is particularly useful for deriving the scaling behaviour of the amplitudes not only with $\delta_{v} \equiv v_{E W} / m_{\chi}$, but also with the Yukawa couplings $y_{f}$ and the gauge coupling $g$. We express the results of this procedure in terms of the ratio of the three-body to the corresponding two-body amplitudes, the latter of which are suppressed by a factor $m_{f}=y_{f} v_{E W}$. If the three-body process lifts Yukawa suppression, the amplitude ratio will thus scale as $\propto 1 / y_{f}$, and if it lifts isospin suppression it scales as $\propto 1 / \delta_{v}$.

We therefore introduce the dimensionless ratio of the helicity amplitudes to the spinsummed/-averaged matrix element for the corresponding two-body process,

$$
R^{\lambda, h} \equiv m_{\chi} \frac{\mathcal{M}^{\lambda, h}}{\sqrt{\mid \overline{\left.\mathcal{M}\right|^{2}}}{ }_{2 \rightarrow 2}} .
$$

For the total amplitude squared, the individual helicity contributions have to be summed over, cf. eq. (A.12). For the sake of our discussion here, we organize this sum in a slightly different way and split it into contributions from final state fermions with equal or opposite chirality (rather than singlet and triplet states), as well as longitudinal and transverse polarizations (like before). We note that, since the external fermions are massless in the limit that we are considering here, helicity coincides with chirality and hence becomes Lorentz invariant. In the back-to-back frame introduced in appendix A.1, the helicity components $h=+-$ and $h=-+$ then correspond to chiralities $f_{R} \bar{f}_{R}$ or $f_{L} \bar{f}_{L}$, respectively, and coincide with the spin-triplet components $(1,+1)$ and $(1,-1)$ discussed there. The helicity combinations $h=++$ and $h=--$ of the fermion pair, on the other hand, correspond to $f_{R} \bar{f}_{L}$ and $f_{L} \bar{f}_{R}$ states, respectively. The sum over these latter two contributions is then equivalent to the sum over the singlet and the remaining triplet states, $(0,0)$ and $(1,0)$ in the notation from above. Altogether, this yields the decomposition

$$
\sum_{\lambda, h}\left|R^{\lambda, h}\right|^{2}=\left(R_{L R / R L}^{L}\right)^{2}+\left(R_{L L / R R}^{L}\right)^{2}+\left(R_{L R / R L}^{T}\right)^{2}+\left(R_{L L / R R}^{T}\right)^{2}
$$

where

$$
\begin{aligned}
\left(R_{L R / R L}^{L}\right)^{2} & \equiv \sum_{\substack{\lambda=0 \\
h=++,--}}\left|R^{\lambda, h}\right|^{2}, \quad\left(R_{L L / R R}^{L}\right)^{2} \equiv \sum_{\substack{\lambda=0 \\
h=+-,-+}}\left|R^{\lambda, h}\right|^{2}, \\
\left(R_{L R / R L}^{T}\right)^{2} & \equiv \sum_{\substack{\lambda= \pm 1 \\
h=++,--}}\left|R^{\lambda, h}\right|^{2}, \quad\left(R_{L L / R R}^{T}\right)^{2} \equiv \sum_{\substack{\lambda= \pm 1 \\
h=+-,-+}}\left|R^{\lambda, h}\right|^{2} .
\end{aligned}
$$


For Higgs final states, the summation over polarizations is absent, and we define the corresponding ratios $R_{L R / R L}$ and $R_{L L / R R}$ analogously, corresponding to $h \bar{f}_{R} f_{L}+h \bar{f}_{L} f_{R}$ and $h \bar{f}_{L} f_{L}+h \bar{f}_{R} f_{R}$ final states, respectively.

We then start from our full result for the helicity amplitudes, using the explicit representations of the generic couplings and mass matrices that appear there, and expand them up to $\mathcal{O}\left(\delta_{v}^{2}\right)$. Note that the limit $\delta_{v} \rightarrow 0$ implies in particular that we expand in the fermion mass $m_{f} \propto y_{f} v_{E W}$ and in gauge boson masses $m_{W / Z} \propto g v_{E W}$. In order to simplify the resulting analytic expressions, we set all sfermion masses equal to the neutralino mass, noting that larger sfermion masses would suppress $t / u$-channel rates relatively strongly because $\sigma v_{2 \rightarrow 3}^{t-\text { channel }} \propto m_{\tilde{f}}^{-8}\left(m_{\tilde{f}}^{-4}\right)$ for Bino- (Higgsino/Wino-)like neutralinos, as discussed previously [31, 33, 36, 37, 92]. Furthermore, we use the notation $B \bar{F} f$ where for neutral bosons ( $B=Z, h)$ the final state fermion types are identical, $\bar{F}=\bar{f}$, while for charged bosons we adopt in the following the convention that $f$ denotes the up-type fermion (e.g. the top quark in $\chi \chi \rightarrow W \bar{b} t)$. In these cases, we keep for simplicity only the dependence on the Yukawa coupling of the up-type fermion, and set the other one to zero.

We furthermore consider six distinct scenarios describing the dominant neutralino composition, which result from different assumptions about the involved mass hierarchies and which are of particular phenomenological interest:

- Higgsino DM, with small Bino admixture $\left(\mu \ll M_{2}, M_{1} \rightarrow \infty\right)$

- Higgsino DM, with small Wino admixture $\left(\mu \ll M_{1}, M_{2} \rightarrow \infty\right)$

- Bino DM, with small Wino admixture $\left(M_{1} \ll M_{2}, \mu \rightarrow \infty\right)$

- Bino DM, with small Higgsino admixture $\left(M_{1} \ll \mu, M_{2} \rightarrow \infty\right)$

- Wino DM, with small Bino admixture $\left(M_{2} \ll M_{1}, \mu \rightarrow \infty\right)$

- Wino DM, with small Higgsino admixture $\left(M_{2} \ll \mu, M_{1} \rightarrow \infty\right)$

From the Bino-, Wino- and Higgsino mass parameters $M_{1}, M_{2}$ and $\mu$, we define the dimensionless mass suppression factors $\delta_{M_{1}} \equiv m_{\chi} / M_{1}, \delta_{M_{2}} \equiv m_{\chi} / M_{2}$ and $\delta_{\mu} \equiv m_{\chi} / \mu$. For all six scenarios listed above, we expand the amplitude ratios to leading order in these mass suppression factors. Effectively, the neutralino mixing between either Bino or Wino and Higgsino then becomes a perturbative 'mass insertion' $\propto g v_{E W}$ represented by the respective off-diagonal entries in the mass matrix of eq. (3.2). Furthermore, for definiteness, we also expand to linear order in $\delta_{A} \equiv m_{\chi} / M_{A}$, i.e. we work in the decoupling limit where the heavy Higgs states are much heavier than the neutralino or SM-like Higgs boson. We note that it is straightforward to generalize these results, and our numerical results anyway include all MSSM Higgs bosons and are valid for arbitrary mass hierarchies.

To lowest order in the expansion parameters defined above, isospin and fermion chirality have to be conserved in all interaction vertices (assuming that the mass splitting between $M_{1}, M_{2}$ and $|\mu|$ is large compared to $\left.g v_{E W}\right)$. One of the implications, as it turns out, is that the gauge-invariant subset of $t$-channel diagrams discussed in section 3.1 can be further split into two separate gauge-invariant sets. The first, which we will denote by 
$(I)$, does not contain any neutralino mixing insertion $\propto g v_{E W}$, and would hence contribute even in the limit of a pure neutralino state. The set of diagrams that contain at least one such insertion (denoted by $(I I)$ ), on the other hand, require a mixing in the neutralino sector (just like is the case for all $s$-channel diagrams).

In tables 9-11, we show the results of this expansion for the helicity-summed ratios $R$ that we have introduced in eq. (A.16), where the different tables correspond to the three types of final states ( $W \bar{F} f, Z \bar{f} f$, and $h \bar{f} f$, respectively). Each table contains the results for all six mass hierarchy scenarios specified above, broken down to contributions from each set of gauge-invariant diagrams. ${ }^{19}$ For the sake of the presentation, we keep only contributions that lift the isospin- or Yukawa suppression of the corresponding two-body process (or both). In particular, as apparent from table 2, the ratio $R_{L R / R L}^{T}$ cannot lift any of these suppressions, and is therefore not included in tables 9-11. Furthermore, for each of the gauge-invariant sets of diagrams, we include only those amplitude ratios that actually do lift at least one of the suppression factors. For the remaining entries, a ' 0 ' indicates three-body amplitudes that vanish to the order we consider, while for entries containing a '-' both 2- and three-body amplitudes vanish. ${ }^{20}$ The two-body amplitudes, finally, are for convenience summarized in table 12 .

\footnotetext{
${ }^{19}$ We checked explicitly (up to $\left.\mathcal{O}\left(\delta_{v}^{2}\right)\right)$ that the Ward identities, eqs. (2.7), (2.8) are satisfied for each set separately. Note that we use Breit-Wigner widths in the amplitudes, and while they break gauge invariance at $\mathcal{O}\left(\delta_{v}\right)$, they do not contribute to the amplitudes as $\delta_{v} \rightarrow 0$.

${ }^{20}$ While in this case the ratio would be formally ill-defined, we only identified one example where the two-body amplitude vanishes while the three-body amplitude does not, marked with a $(*)$. We note that the relevant process, $\chi \chi \rightarrow W W^{*} \rightarrow W \bar{F} f$ for a Wino-like neutralino, is phenomenologically not important because for $m_{\chi}>M_{W}$ it is largely captured by annihilation into $W W$, while Wino-like neutralinos with $m_{\chi}<M_{W}$ are practically excluded.
} 


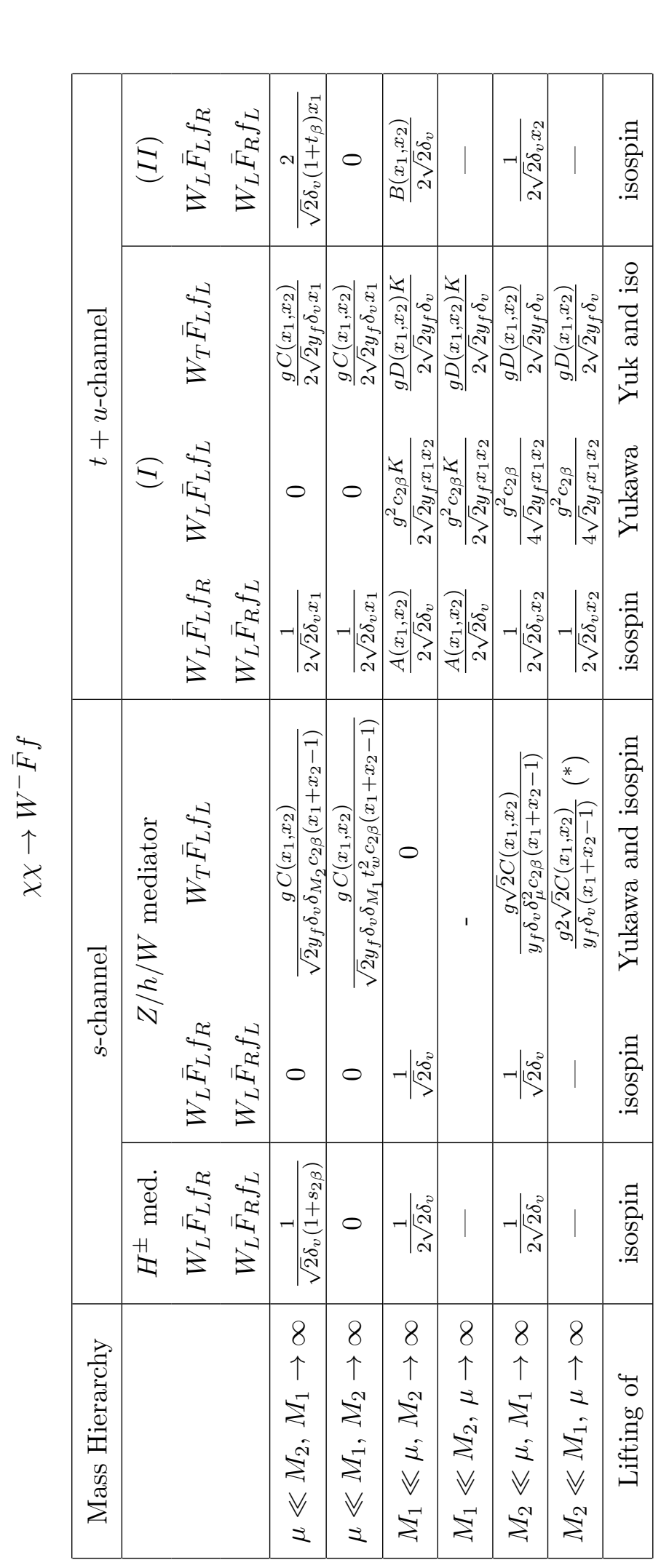

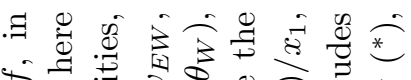

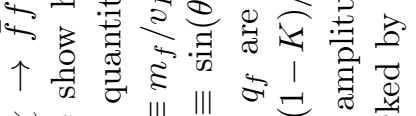

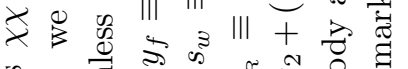

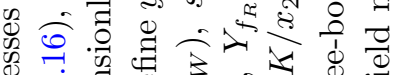

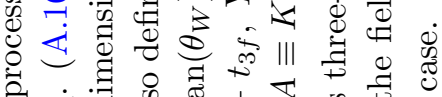

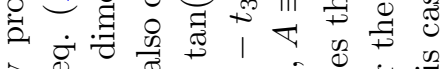
B. 0 व 영

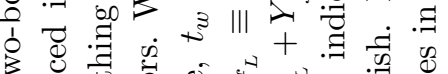

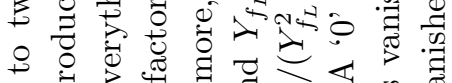
ธ.

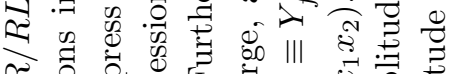

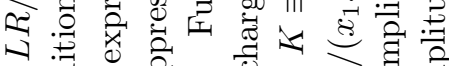

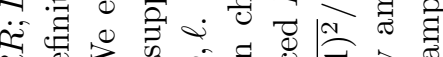

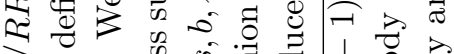

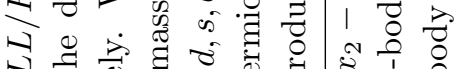
$\checkmark \exists$ $\|$ 원

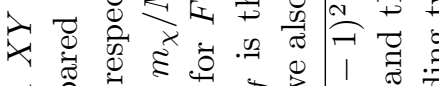

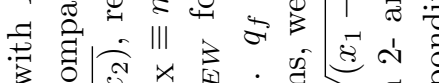

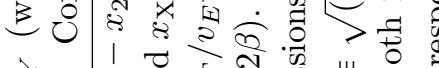
« It)

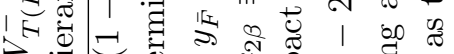

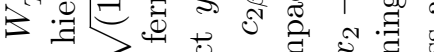
$\uparrow$ 出 ૪

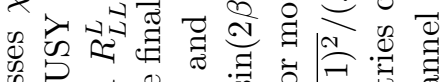

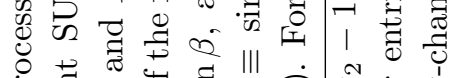

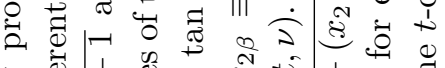

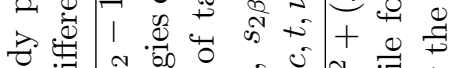

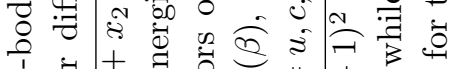

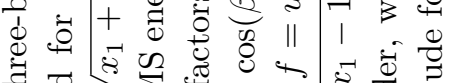

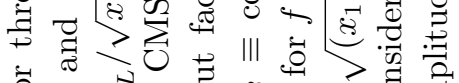

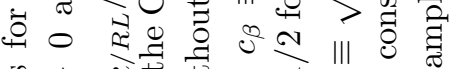

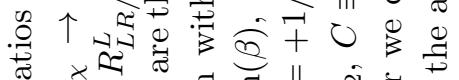

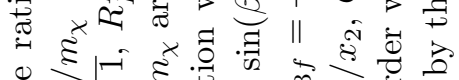

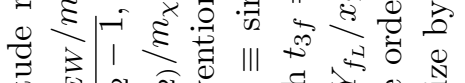

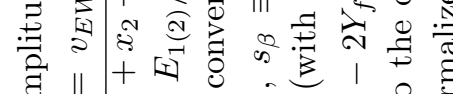

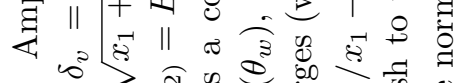
$0 . \frac{1}{3}$

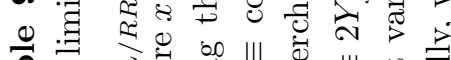

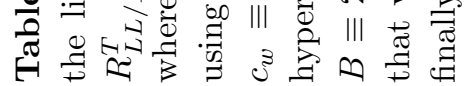




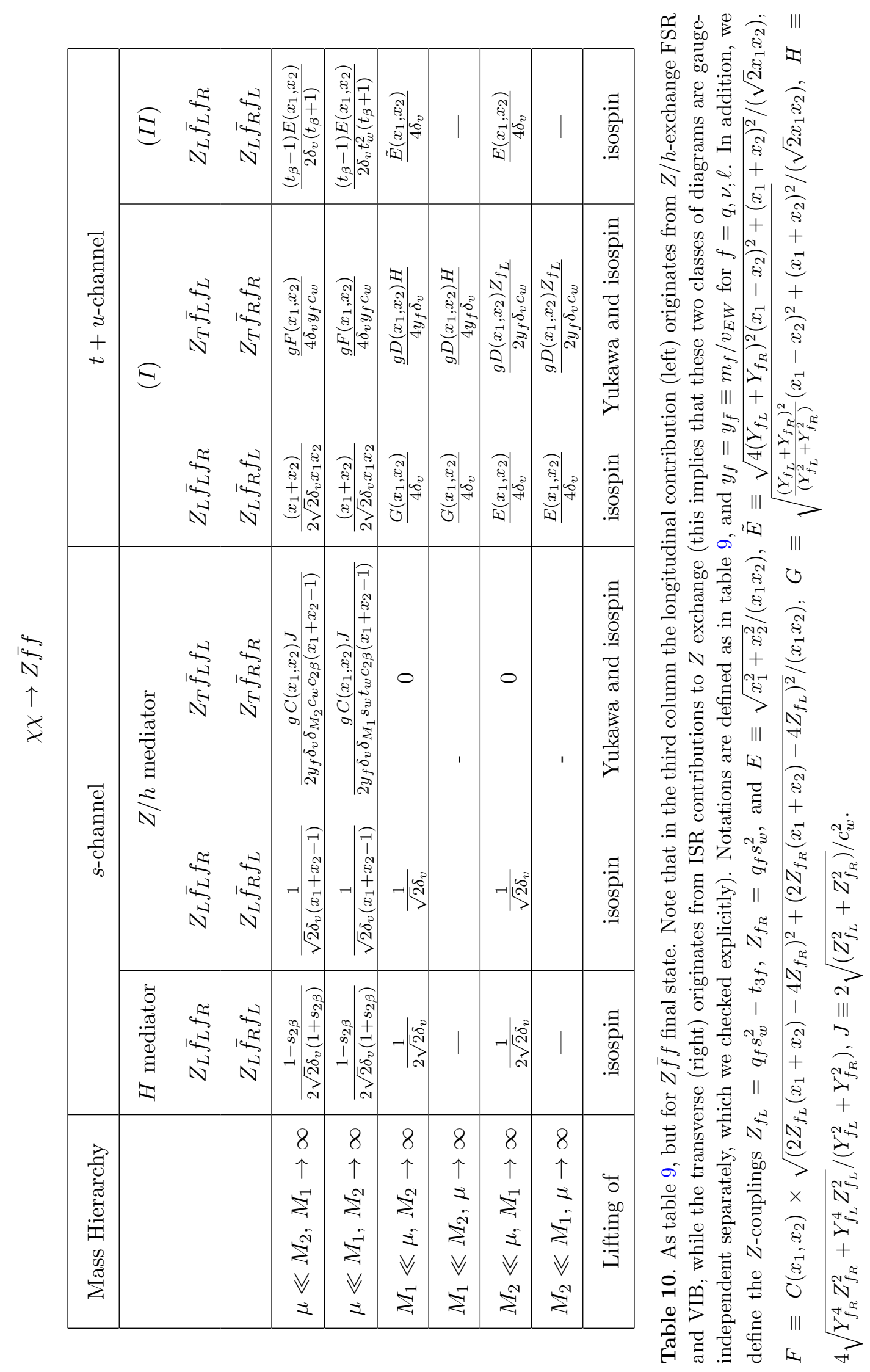




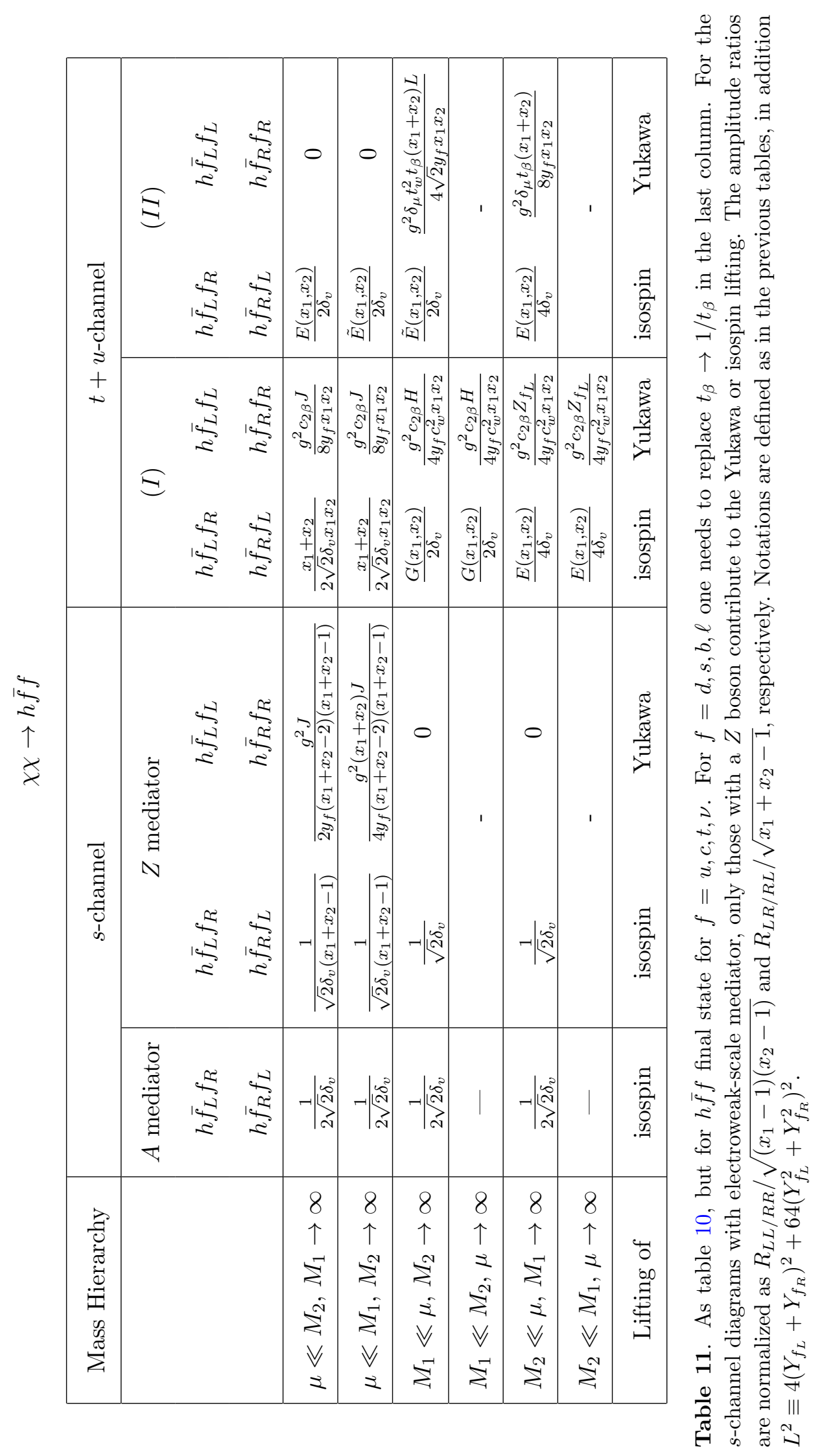




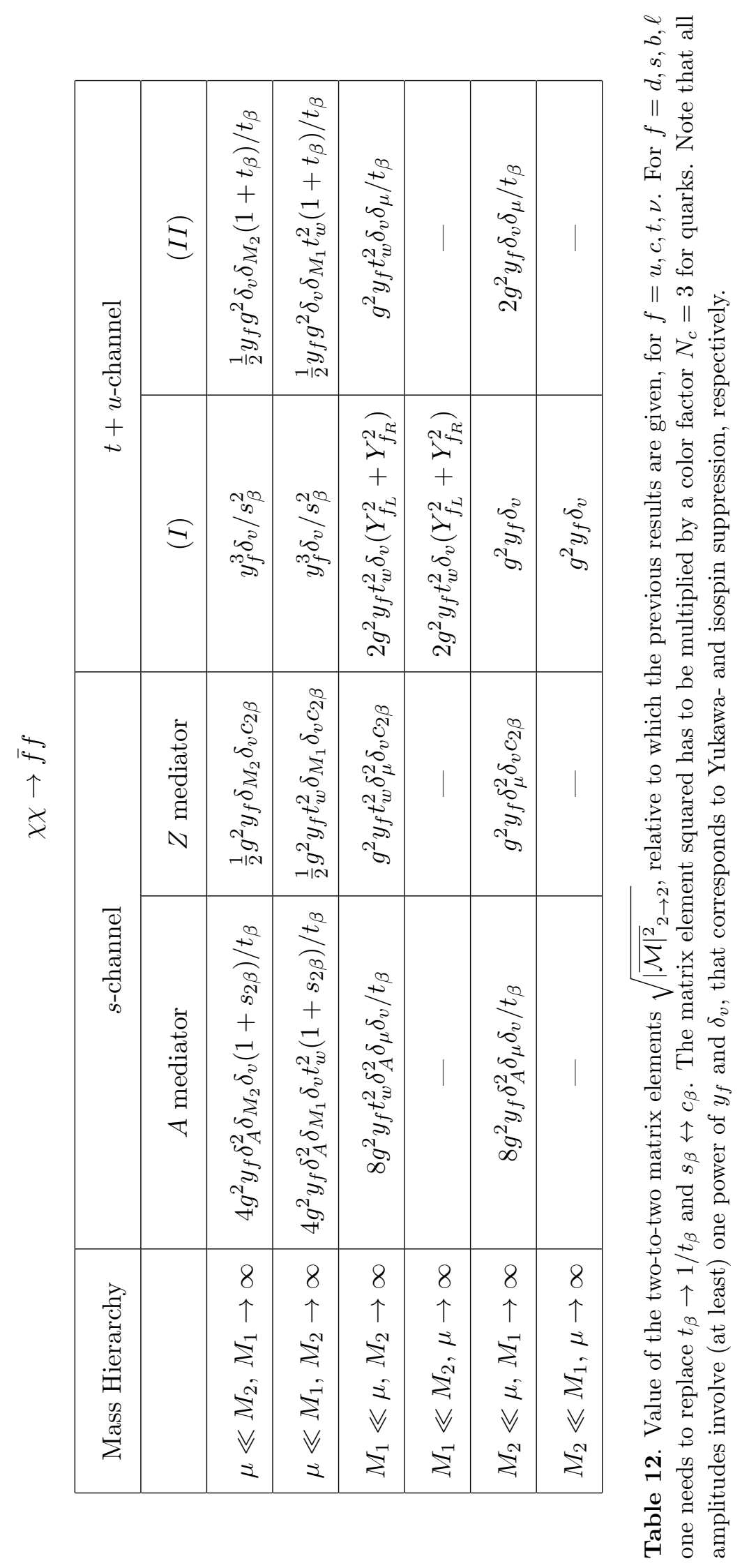


In order to assess the parametric enhancement of three-body over two-body processes, it is sufficient to consider the amplitude ratios just presented, and we will continue with a more detailed discussion of the various lifting mechanisms at the level of individual diagrams in the following subsection A.3. Before doing so, let us briefly remark that the corresponding cross section ratio for $\chi \chi \rightarrow B \bar{F} f$, normalized to the one for $\chi \chi \rightarrow \bar{f} f$, is obtained by

$$
\frac{1}{\sigma v_{2 \rightarrow 2}} d(\sigma v)_{2 \rightarrow 3}=\frac{1}{4 \pi^{2}} \frac{1}{\sqrt{1-m_{f}^{2} / m_{\chi}^{2}}} \sum_{\lambda, h}\left|R^{\lambda, h}\right|^{2} d x_{1} d x_{2}
$$

where $x_{i}=E_{i} / m_{\chi}$ are the dimension-less fermion energies of the three-body final state. Using the results from tables 9-11, one can thus obtain the contribution to this ratio from each of the gauge-invariant subsets of diagrams separately. In the limit of massless final state particles, the integration ranges are $0<x_{1}<1$ and $1-x_{1}<x_{2}<1$, implying that some of these integrations become logarithmically divergent. This is an expected artefact of the expansion in $\delta_{v}$ and, in practice, the corresponding infrared divergent contributions are cut off by the non-zero mass of the vector boson. Throughout this work, we assume that the resulting logarithmic enhancement $\mathcal{O}\left(\frac{\alpha}{\pi} \ln ^{2}\left(E_{B} / \sqrt{s}\right)\right)$ can be treated perturbatively down to the infrared cutoff $E_{B} \sim m_{B} \sim g v_{E W}$. This imposes an upper limit on the neutralino mass of roughly $m_{\chi} \ll \mathcal{O}\left(g v_{E W} e^{\pi / g}\right) \sim \mathcal{O}(10) \mathrm{TeV}$. If one is interested in higher masses, it would be interesting to apply the resummation methods discussed e.g. in refs. [55-57]. On the other hand, we stress that the logarithmic sensitivity to $\ln ^{2}\left(g \delta_{v}\right)$ does not spoil the power counting arguments related to lifting of isospin suppression factors, since the latter is described by powers $\delta_{v}^{n}$ of $\delta_{v}$. In our numerical results, we fully take into account the masses of all annihilation products.

\section{A.3 Suppression lifting from individual diagrams}

It is rather illustrative to reflect the results of the previous subsection at the level of individual diagrams. In table 3, displayed for clarity already in the main text (see section 3.3), we therefore organize all relevant amplitudes in a large table, with the four rows corresponding to the four gauge-invariant subsets. For each type of diagram, and assuming a Bino- or Wino-like neutralino, we furthermore explicitly indicate the scaling with the gauge coupling $g$, the Yukawa coupling $y_{f}$, and the vev $v_{E W}$ (we comment on the Higgsino-like case below). Let us start our discussion with the first column, which contains the diagrams contributing to the two-body process $\chi \chi \rightarrow \bar{f} f$. As expected, all these amplitudes scale as $\propto g^{2} y_{f} v_{E W}$, but the origin differs:

$\boldsymbol{t}$-channel $\boldsymbol{I}$. The factor $y_{f} v_{E W}$ enters either via the chirality flip of one of the final-state fermions, or via a $L / R$ mixing insertion of the sfermion (for brevity, we show only one representative diagram in table 3 for each of these cases).

$\boldsymbol{t}$-channel $\boldsymbol{I I}$. The factor $v_{E W}$ enters via the gaugino/Higgsino mixing insertion on one of the initial lines, and the Yukawa suppression enters via the Higgsino-sfermion-fermion coupling. 
$s$-channel EW. The $s$-channel with electroweak-scale mediator corresponds to the $Z$ exchange diagram mentioned earlier. In the $s$-wave limit, and from the perspective of the unbroken theory, this diagram is represented by the exchange of the pseudoscalar Goldstone boson $G^{0}$. The factor $v_{E W}$ arises from the gaugino/Higgsino mixing, and the Yukawa coupling from the Yukawa interaction $G^{0} \bar{f} f$.

$\boldsymbol{s}$-channel $\boldsymbol{M}_{\boldsymbol{A}}$. This case is similar to the previous one, except that the mediator is replaced by the (physical) heavy pseudoscalar Higgs $A$.

Let us now turn our discussion to the remaining columns of table 3, which contain all relevant three-body processes. Here, the second column shows representative Feynman diagrams that lead to a lifting of both isospin and Yukawa suppression, while the third and fourth column show diagrams that lift only one of them, respectively:

Lifting of Yukawa and isospin suppression. Both suppression factors can be lifted only for two of the gauge-invariant sets of diagrams $(t-I$ and $s$-EW). In the former case, a transverse $Z_{T}$ or $W_{T}$ is emitted from either fermion line in the final state, from the sfermion line, or from the initial lines (this last case cannot occur in the Bino-like case). We remark that FSR can only lift the helicity suppression if the virtual fermion is strongly off-shell, i.e. not for soft and collinear photons (which are sometimes defined as FSR, see footnote 4). In the $s$-channel case, the diagrams can be thought of as an annihilation $\chi \chi \rightarrow W W^{*}$, with subsequent decay of $W^{*}$ (see section 3.5 for a discussion of such off-shell internal states). It is impossible to lift both suppression factors for the other two classes: for $t-I I$, this would require a gaugino-Higgsino- $W / Z$ vertex, which is absent for $v_{E W} \rightarrow 0$. The same applies for $s-M_{A}$, noting in addition that the $A \bar{f} f$ coupling requires also the presence of a Yukawa coupling.

Lifting of only isospin suppression. The isospin suppression can be lifted for all four subsets, by replacing the insertion of $v_{E W}$ within the two-body amplitude by the emission of a Higgs boson or a Goldstone boson, respectively. Note that for the set $t-I$ this amounts to replacing the fermion mass insertion by a fermion-fermion-Higgs/Goldstone coupling (or replacing the sfermion $L / R$ mixing insertion by a sfermion-sfermion-Higgs/Goldstone coupling, respectively). For all other sets one replaces the gaugino-Higgsino mixing insertion in the initial line by a gaugino-Higgsino-Higgs/Goldstone vertex. For the $s$-channel, the diagrams can also be thought of as an annihilation into a pair of scalars, with subsequent decay of one of them. This mechanism of suppression lifting is very general, and appears for all gauge invariant subsets of diagrams as well as for all final states (involving $W / Z$ or a Higgs boson). We expect it to be relevant especially for heavy neutralino masses.

Lifting of only Yukawa suppression. This case is in some sense the most difficult to realize. The reason is that it requires a Higgs (or Goldstone) boson in the final state, and therefore only diagrams where the final-state boson does not couple directly to the final-state fermions can potentially contribute in the limit $y_{f} \rightarrow 0$. We identified three such processes, shown in the last column in table 3: for $t-I$, the Higgs (or charged 
Goldstone boson; note that there is no sfermion-sfermion- $G^{0}$ vertex for $y_{f} \rightarrow 0$ ) can be emitted from the sfermion line in the $t$-channel, i.e. via VIB. The corresponding vertex is derived from a four-scalar sfermion-sfermion-Higgs-Higgs interaction, involving the full Higgs doublets. This coupling leads to the required vertices at $\mathcal{O}\left(v_{E W}\right)$, and scales with $g^{2}$ for $y_{f} \rightarrow 0$ within the MSSM (see refs. [33] and [20] for a discussion within a toy model for the Goldstone- and Higgs-emission, respectively). In addition, for $t-I I$, the Higgs can be emitted via ISR (second row, last column of table 3). While this contribution lifts Yukawa suppression, it is suppressed compared to the two-body process for a large mass hierarchy between gaugino and Higgsino mass parameters; we nevertheless kept this contribution, because the former effect can easily compensate for the latter. Finally, for the $s$-EW case, the Higgs can be emitted from the $s$-channel mediator via a Goldstone-Higgs- $Z$ coupling (third row, last column in table 3). Note that this mechanism is distinct from the one discussed in [38], and that the toy-model discussed there cannot be realized within the MSSM. To the best of our knowledge, both the $t$-channel ISR and the $s$-channel Higgstrahlung processes that we identified within the MSSM have not been discussed before.

One can understand the diagrams that lift Yukawa or isospin suppression as shown in table 3 based on basic properties of the unbroken MSSM Lagrangian, as well as the symmetry requirement $J_{C P}=0_{-}$of the $s$-wave initial state. For example, mixing insertions $\propto g v_{E W}$ of the neutralino line can turn a Bino into a Higgsino, but not into a Wino. In addition, the Higgsino coupling to fermion/sfermion pairs is proportional to the Yukawa coupling, while the corresponding coupling for Bino- and Wino-like neutralinos involves a gauge coupling and is therefore generally much less suppressed (except for the top quark). One slightly more involved example is the diagram in the last column of the first row. For final states involving a longitudinal $W_{L}$, the corresponding sfermion vertex derives from the interaction term $\propto g^{2}\left(\tilde{f}_{L}^{\dagger} H\right)\left(H^{\dagger} \tilde{f}_{L}\right)$ present for sfermion fields that transform as doublet under $\mathrm{SU}(2)_{L}$. After inserting the decomposition $H=\left(G^{+},\left(v_{E W}+h+i G^{0} / \sqrt{2}\right)\right)$ of the SM-like Higgs doublet one easily verifies that at linear order in $v_{E W}$ one obtains a sfermion coupling to $G^{ \pm}$and $h$, but not to $G^{0}$, which explains why no longitudinal $Z_{L}$ boson can be produced in this case. The Higgs final state also receives a further contribution from the interaction term $\propto H^{\dagger} H \tilde{f}^{\dagger} \tilde{f}$, which exists for all (left and right) sfermion fields. Furthermore, for the $s$-channel processes of the type $\chi \chi \rightarrow h B^{*} \rightarrow h \bar{f} f$ that give a non-zero contribution in the $s$-wave limit, the mediator $B$ is a pseudoscalar or transverse vector (i.e. $G^{0}, A^{0}, Z_{T}$ ), while for $\chi \chi \rightarrow G^{0} B^{*} \rightarrow G^{0} \bar{f} f, B$ is a scalar (i.e. $h, H^{0}$ ). This is consistent with the odd $C P$ parity of the initial state.

Note that the above arguments are only valid when expanding around the unbroken theory, and representing longitudinal degrees of freedom by Goldstone bosons. In fact, within the broken theory, analogous arguments would be hampered by large cancellations that occur among individual diagrams, and that make the power counting less transparent. Nevertheless, we carefully cross checked that all these arguments can indeed be reproduced when using the full matrix elements within the broken theory, and expanding the sum of all diagrams within a gauge invariant subset for heavy neutralino mass. 
While the discussion above assumed a gaugino-like neutralino, the case of a Higgsinolike neutralino is very similar. For the third and fourth row in table 3, in particular, nothing changes except that the incoming neutralino is now a Higgsino in the limit $v_{E W} \rightarrow 0$, and the insertion $\propto g v_{E W}$ denotes mixing with either a Bino or Wino (in addition, both $Z_{T}$ and $W_{T}$ ISR is possible, while only $W_{T}$ ISR is possible for Wino-like neutralinos). The same applies to the second line, after interchanging the label of $g$ and $y_{f}$ on the vertices involving a sfermion in all diagrams in the first and second column (this does not affect the overall scaling of the amplitude), while the diagram in the last column would receive an additional $y_{f}^{2}$ suppression. For the first row, the two neutralino-sfermion-fermion vertices scale with $y_{f}$ instead of $g$ in all diagrams. Thus, this class is additionally suppressed by a factor $y_{f}^{2}$ compared to the other subsets. Nevertheless, for completeness, we kept this case because the three-body processes can lift the additional suppression factors $y_{f} v_{E W}$ of the two-body amplitude in the same way as for a gaugino-like neutralino.

In summary, we confirmed the general symmetry arguments outlined in section 2.2 for the MSSM and explicitly identified the contributions to the three-body amplitudes that realize the suppression lifting, focussing on final states containing (tranverse or longitudinal) gauge bosons as well as the SM-like Higgs boson. By expanding the full amplitudes in various limits that correspond to Bino-, Wino- or Higgsino-like neutralino, respectively, we find that (almost) all of the possibilities allowed by symmetries are realized. The cases for which we did not find a contribution within the MSSM are marked by a '-' in table 2. For processes involving $W$ bosons and purely right-handed fermions an additional suppression arises that can be traced back to the chiral structure of the $\mathrm{SU}(2)_{L}$ interaction. For processes involving $Z_{L}$ (represented by $G^{0}$ ) or $A$, and fermions of equal chirality, on the other hand, lifting of Yukawa suppression would require that the amplitude does not contain Yukawa interaction vertices. In addition, vertices such as sfermion-sfermion- $G^{0} / A$ are absent for $y_{f} \rightarrow 0$ (as required by $C P$-invariance), such that a $t$-channel process analogous to the one in the first row, last column of figure 3 does not exist. For the $s$-channel, the symmetries of the initial state would require a CP-even mediator if the $G^{0}$ or $A$ was emitted via ISR. Within the MSSM, only the Higgs bosons are available. However, their coupling to fermions necessarily involve a Yukawa coupling, such that Yukawa suppression cannot be lifted in this specific process. Similarly, one can convince oneself that the $s$-channel VIB process (3rd row, 4th column of figure 3) as well as the remaining $t$-channel process (2nd row, 4th column) cannot occur when replacing $h \rightarrow G^{0}, A$.

\section{B Numerical implementation}

For each Feynman diagram, we have implemented the full analytical expressions for the helicity amplitudes in DarkSUSY [40]. We numerically sum over these contributions to obtain the total amplitude for a given helicity configuration, $\mathcal{M}_{\chi \chi \rightarrow \bar{F} f X}^{(h, \lambda)}$, as introduced in appendix A.1. Differential and partial cross sections are computed according to eq. (A.13), by numerically integrating over the energies of the final state particles; for consistency checks, this can be done for any pair of energies and in any specified order. In order to 
improve convergence and accuracy of the numerical integrations, we use taylored integration routines that make use of the known locations of kinematic resonances [87].

For the total cross sections, we have explicitly implemented the NWA approximations contained in eqs. (4.3)-(4.9). We have extensively checked our code, and hence also the prescription of subtracting the NWA contribution detailed above, by comparing the total cross section defined in eq. (4.10), on a channel-by-channel basis and for various SUSY models, with numerical results obtained with CalcHEP [93]. ${ }^{21}$ For all models, and all annihilation channels, we find remarkable agreement. We also checked agreement for individual classes of diagrams $(s / t u$-channel, ISR/FSR/VIB) as classified in section 3.1. Let us stress that in terms of computation time the implementation via helicity amplitudes, together with the taylored integration routines, is less expensive compared to the evaluation of squared matrix elements via Monte Carlo integration as implemented in CalcHEP. This is especially significant for the three-body processes to which a large number of diagrams contribute, and for which the difference in computation times amounts to several orders of magnitude in the specific kinematic limit we are interested in here.

For the yields of stable particles, we have implemented the procedure described in section 4.2, using unpolarized yields for decaying particles given that these are the only ones that are currently available in DarkSUSY [95]. As discussed, as long as the total yields (i.e. summed over all channels) are concerned, our prescription still captures any double counting. We note that extending our implementation to fully polarized yields will be straight-forward for future work, given the results provided in section 4.2 and the helicity amplitudes reported in appendix A.

Let us mention a few of the extensive numerical checks that we performed to test the yield implementation. We considered, in particular, models for which the three-body annihilation is dominated by an almost on-shell intermediate resonance. In this case, the

\footnotetext{
${ }^{21}$ We compared our implementation of three-body cross sections based on DarkSUSY 5.1 with CalcHEP 3.4 [93]. In particular, we adapted the ewsbMSSM implementation of CalcHEP to compute the spectrum from a given set of pMSSM input parameters at scale $Q=M_{Z}$ (except for $M_{A}$ which is the pole mass) using SoftSusy 3.4 [94]. The Susy les Houches output file written by SoftSusy is then used as input for DarkSUSY via the slha interface. In order to be able to directly compare the output it is necessary to adapt various routines in order to match the conventions. Apart from making sure that all SM input parameters agree (we used $m_{b}=4.92 \mathrm{GeV}, \sin \left(\theta_{W}\right)=0.47162, \Gamma_{W}=2.07 \mathrm{GeV}, \Gamma_{t}=2.0 \mathrm{GeV}$ ), we made the following changes for the purpose of cross checking: for CalcHEP, we switched off the running bottom mass $(\mathrm{dMbOn}=0)$ and used unitary gauge (for the comparison on a diagram-by-diagram basis; only the sum is gauge-independent). For DarkSUSY, the Yukawa couplings are by default read in from the blocks YU, YE and YD in the slha file. For the purpose of comparison, it is convenient to fix the Yukawa couplings at $y_{i}=m_{i} / v$, especially for the top. Therefore, we commented out the corresponding lines in dsfromslha.f. Additionally, in su/dssuconst_yukawa_running.f, we commented out the running Yukawas, such that the default Yukawa couplings, which are simply related to the (on-shell) masses, are used. In addition, the call to dshigwid() was commented out in dsfomslha.f in order to avoid a rescaling of Higgs couplings that takes certain NLO corrections into account. For the purpose of comparison, it is more convenient to have tree-level couplings. In addition, we then set the Higgs widths to a common value in both programs. Finally, we set the first and second generation quark masses to zero and the CKM mixing matrix to unity in order to match the conventions of the ewsbMSSM model implemented in CalcHEP. We verified that the conventions agree by comparing also the two-body cross sections for all channels allowed at $s$-wave, for which we find perfect agreement after the changes described above.
} 
subtraction procedure described in section 4.2 is expected to lead to a large cancellation between the full three-body contribution and the NWA term. We explicitly verified this cancellation for all yields of stable particles, and over the full energy range. The cancellation amounts to several orders of magnitude in specific cases, and therefore provides a robust check of the implementation. In addition, we also verified that the yields obtained from all of the models contained in our MSSM scan results pass a number of checks (e.g. yields within an expected range at $E>m_{\chi} / 2$ and $\left.E>m_{\chi} / 10\right)$. Finally, we also considered three-body final states that contain directly one or more stable particles (such as e.g. $\chi \chi \rightarrow W e \nu)$. In this case, we verified that the neutrino and positron spectra match the analytical result discussed in appendix $\mathrm{C}$ for specific models for which this final state is dominantly produced by an intermediate $W$ resonance.

\section{Spin correlations of decaying resonances}

In section 4, we discussed how to subtract double counting due to on-shell intermediate states ('resonances') contributing to three-body annihilation processes. If the resonance carries a spin, the spectrum of final state particles depends on how much the various helicity states of the resonance contribute. In section 4 we argued that for annihilation of Majorana fermions in the $s$-wave limit, $\mathrm{CP}$ and angular momentum conservation uniquely determine the helicity of all possible intermediate states that can contribute to the threebody processes considered here. Here we present a formal derivation of this result, based on a description that would in principle allow us to treat also more general cases.

In full generality, several helicity states of the resonance contribute to the amplitude, and can also interfere with each other when taking the absolute value squared. As a starting point we consider the example $\chi \chi \rightarrow H W \rightarrow H f \bar{F}$. We are interested in the contribution from the on-shell intermediate $W$ boson. The full matrix element squared can then be written in the form

$$
{\overline{\left|\mathcal{M}_{\mathrm{res}}\right|}}^{2} \equiv \sum_{s 1, s 2}\left|\sum_{\lambda} \mathcal{M}_{2 \rightarrow 2}^{\mu} \epsilon_{\mu}^{* \lambda} \epsilon_{\nu}^{\lambda}\left(\mathcal{M}_{s 1, s 2}^{\nu}\right)_{1 \rightarrow 2}\right|^{2},
$$

where we indicated explicitly the summation over the final-state spins of the fermions, and the polarization states of the internal $W$. To extract the on-shell contribution in the narrow-width limit we assume that the momentum $q_{\mu}$ of the $W$ is (almost) on-shell, $q^{2} \simeq M_{W}^{2}$. This implies that the kinematics of the $H$ and $W$ momenta is identical to the two-body annihilation. The first term inside the square contains the helicity amplitude for the two-body part,

$$
\mathcal{M}_{2 \rightarrow 2}^{\lambda} \equiv \mathcal{M}_{2 \rightarrow 2}^{\mu} \epsilon_{\mu}^{* \lambda}
$$

For concreteness we can take the momentum of the $W$ to be along the z-axis, $q_{\mu}=$ $\left(E_{q}, 0,0,|q|\right)$, where $E_{q}=\sqrt{|q|^{2}+M_{W}^{2}}$ and $|q|$ is determined by the neutralino, W and Higgs mass via the two-body kinematics (identical to $\left|p_{3}\right|$, see (C.22) below). The decay $W \rightarrow f \bar{F}$ gives (fermion momenta $p_{1}$ and $p_{2}$ )

$$
\left(\mathcal{M}_{s 1, s 2}^{\nu}\right)_{1 \rightarrow 2}=\bar{u}_{s_{1}}\left(p_{1}\right)\left(g P_{L} \gamma^{\nu}\right) v_{s_{2}}\left(p_{2}\right)
$$


Inserting these into the resonant matrix element, and writing out the square gives after some renaming of indices

$$
\begin{aligned}
{\overline{\mid \mathcal{M}_{\mathrm{res}}}}^{2} & =\sum_{\lambda, \lambda^{\prime}} \mathcal{M}_{2 \rightarrow 2}^{* \lambda^{\prime}} \mathcal{M}_{2 \rightarrow 2}^{\lambda} \epsilon_{\mu}^{* \lambda^{\prime}} \epsilon_{\nu}^{\lambda} \sum_{s_{1}, s_{2}}\left(\mathcal{M}_{s_{1} s_{2}}^{* \mu}\right)_{1 \rightarrow 2}\left(\mathcal{M}_{s_{1} s_{2}}^{\nu}\right)_{1 \rightarrow 2} \\
& =\sum_{\lambda, \lambda^{\prime}} \mathcal{M}_{2 \rightarrow 2}^{* \lambda^{\prime}} \mathcal{M}_{2 \rightarrow 2}^{\lambda} \times \mathcal{D}_{\lambda \lambda^{\prime}}
\end{aligned}
$$

where

$$
\begin{aligned}
\mathcal{D}_{\lambda \lambda^{\prime}} & \equiv \epsilon_{\mu}^{* \lambda^{\prime}} \epsilon_{\nu}^{\lambda} \sum_{s_{1}, s_{2}}\left(\mathcal{M}_{s_{1} s_{2}}^{* \mu}\right)_{1 \rightarrow 2}\left(\mathcal{M}_{d, s_{1} s_{2}}^{\nu}\right)_{1 \rightarrow 2} \\
& =\epsilon_{\mu}^{* \lambda^{\prime}} \epsilon_{\nu}^{\lambda} g^{2} \operatorname{tr}\left(\left(p_{2}-m_{F}\right) \gamma_{\mu} P_{R}\left(p p_{1}+m_{f}\right) P_{L} \gamma_{\nu}\right) \\
& =\epsilon_{\mu}^{* \lambda^{\prime}} \epsilon_{\nu}^{\lambda} 2 g^{2}\left(p_{2}^{\mu} p_{1}^{\nu}-g^{\mu \nu} p_{1} \cdot p_{2}+p_{2}^{\nu} p_{1}^{\mu}+i \epsilon^{\mu \nu \kappa \rho} p_{2 \kappa} p_{1 \rho}\right)
\end{aligned}
$$

The decorrelation-approximation (i.e. the replacement of the matrix element by the product of two matrix elements with independent summation over spin states, (3.6)) would be obtained by replacing

$$
\mathcal{D}_{\lambda \lambda^{\prime}} \rightarrow \mathcal{D}_{\lambda \lambda^{\prime}}^{\text {decorrelated }} \equiv \frac{1}{3} \delta_{\lambda \lambda^{\prime}} \sum_{\lambda^{\prime \prime}} \mathcal{D}_{\lambda^{\prime \prime} \lambda^{\prime \prime}}
$$

Instead, one can also try to use the full matrix $\mathcal{D}_{\lambda \lambda^{\prime}}$. To compute it, one can use the helicity basis

$$
\epsilon_{\mu}^{ \pm}=(0,1, \pm i, 0) / \sqrt{2}, \quad \epsilon_{\mu}^{0}=\left(|q|, 0,0, E_{q}\right) / M_{W}
$$

which fulfill $\epsilon_{\mu}^{* \lambda^{\prime}} \epsilon^{\mu \lambda}=-\delta_{\lambda \lambda^{\prime}}$ as well as $\sum_{\lambda} \epsilon_{\mu}^{* \lambda} \epsilon_{\nu}^{\lambda}=-g_{\mu \nu}+q_{\mu} q_{\nu} / M_{W}^{2}$.

A convenient frame to evaluate it is the rest frame of the $W$, obtained by boosting along the $z$ direction. In this frame $\epsilon_{\mu}^{0}=(0,0,0,1)$. The momenta of the fermions can be parameterized by the angle w.r.t. to the $\mathrm{z}$-axis (which is singled out as the polarization axis of the $W$ ),

$$
p_{1}=\left(E_{p_{1}}^{*}, 0,\left|p_{1}^{*}\right| \sin \theta,\left|p_{1}^{*}\right| \cos \theta\right), \quad p_{2}=\left(M_{W}-E_{p_{1}}^{*}, 0,-\left|p_{1}^{*}\right| \sin \theta,-\left|p_{1}^{*}\right| \cos \theta\right)
$$

where $\left|p_{1}^{*}\right|$ and $E_{p_{1}}^{*}=\sqrt{\left|p_{1}^{*}\right|^{2}+m_{f}^{2}}$ are the momentum and energy of $f$ in the $W$ rest frame, see (C.22). Inserting this and evaluating the trace yields an explicit expression for $\mathcal{D}_{\lambda \lambda^{\prime}}=\mathcal{D}_{\lambda \lambda^{\prime}}(\theta)$ in terms of $\theta$. Using that the polarization vectors have zero temporal component in the basis we are working in, and that $\vec{p}_{2}=-\vec{p}_{1}$,

$$
\mathcal{D}_{\lambda \lambda^{\prime}}(\theta)=2 g^{2}\left(-2\left(\vec{\epsilon}_{\lambda^{\prime}} \vec{p}_{1}\right)^{*}\left(\vec{\epsilon}_{\lambda} \vec{p}_{1}\right)+\delta_{\lambda \lambda^{\prime}} p_{1} \cdot p_{2}+i M_{W}\left(\vec{\epsilon}_{\lambda^{\prime}}^{*} \times \vec{\epsilon}_{\lambda}\right) \cdot \vec{p}_{1}\right)
$$

Now one can use $p_{1} \cdot p_{2}=\left(M_{W}^{2}-m_{f}^{2}-m_{F}^{2}\right) / 2$ and

$$
\begin{aligned}
\vec{\epsilon}_{0} \vec{p}_{1} & =\left|p_{1}^{*}\right| \cos \theta, \quad \vec{\epsilon}_{ \pm} \vec{p}_{1}= \pm i \frac{\left|p_{1}^{*}\right|}{\sqrt{2}} \sin \theta \\
\vec{\epsilon}_{+}^{*} \times \vec{\epsilon}_{+} & =+i \vec{\epsilon}_{0}, \quad \vec{\epsilon}_{-}^{*} \times \vec{\epsilon}_{-}=-i \vec{\epsilon}_{0}, \quad \vec{\epsilon}_{0}^{*} \times \vec{\epsilon}_{ \pm}=\mp i \vec{\epsilon}_{ \pm}, \quad \vec{\epsilon}_{ \pm}^{*} \times \vec{\epsilon}_{0}=\mp i \vec{\epsilon}_{\mp}
\end{aligned}
$$


The result is

$$
\begin{aligned}
\mathcal{D}_{00} & =2 g^{2}\left(p_{1} \cdot p_{2}-2\left|p_{1}^{*}\right|^{2} \cos ^{2} \theta\right) \\
\mathcal{D}_{ \pm \pm} & =2 g^{2}\left(p_{1} \cdot p_{2}-\left|p_{1}^{*}\right|^{2} \sin ^{2} \theta \mp M_{W}\left|p_{1}^{*}\right| \cos \theta\right) \\
\mathcal{D}_{ \pm \mp} & =2 g^{2}\left|p_{1}^{*}\right|^{2} \sin ^{2} \theta \\
\mathcal{D}_{ \pm 0} & =-i \sqrt{2} g^{2}\left|p_{1}^{*}\right| \sin \theta\left(M_{W} \mp 2\left|p_{1}^{*}\right| \cos \theta\right)=\mathcal{D}_{0 \pm}^{*}
\end{aligned}
$$

One can check that the average over the diagonal contributions corresponds to the usual unpolarized decay matrix element,

$$
\overline{\left|\mathcal{M}_{1 \rightarrow 2}\right|^{2}}=\frac{1}{3} \sum_{\lambda} \mathcal{D}_{\lambda \lambda}=\frac{1}{3} g^{2}\left(6 p_{1} \cdot p_{2}-4\left|p_{1}^{*}\right|^{2}\right)=g^{2}\left(M_{W}^{2}-m_{f}^{2}-m_{F}^{2}-\frac{4}{3}\left|p_{1}^{*}\right|^{2}\right)
$$

To obtain the diff. cross section, we use the representation of the phase space in the form

$$
d\left(\sigma v_{H f \bar{F}}\right)=d \Phi \frac{\overline{|\mathcal{M}|}^{2}}{4 M_{\chi}^{2}}=\left.\frac{1}{(2 \pi)^{5}} \frac{1}{16\left(2 M_{\chi}\right)^{2}} \overline{|\mathcal{M}|}\right|^{2}\left|p_{1}^{*}\right|\left|p_{3}\right| d m_{12} d \Omega_{1}^{*} d \Omega_{3}
$$

where $p_{3}$ is the Higgs momentum, and $m_{12}^{2}=q^{2}$ the resonant momentum. Now we can do an approximation where we replace

$$
\overline{\mid \mathcal{M}}^{2} \rightarrow{\overline{\mid \mathcal{M}_{\mathrm{res}}}}^{2} \frac{\pi}{M_{W} \Gamma_{W}} \delta\left(q^{2}-M_{W}^{2}\right)
$$

but keep the fully correlated matrix element ${\overline{\mid \mathcal{M}_{\text {res }}}}^{2}$. By integrating over $d m_{12}=$ $d m_{12}^{2} /\left(2 m_{12}\right)=d q^{2} /\left(2 M_{W}\right)$, and doing the trivial Higgs angle $d \Omega_{3}$ and $d \phi_{1}^{*}$ integrals, one obtains the diff. cross section w.r.t. to the angle $\theta$ of the fermion $f$ and the polarization axis of the $\mathrm{W}$ boson in the back-to-back system,

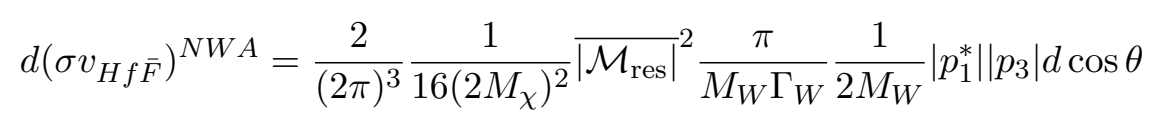

where

$$
\begin{aligned}
& \left|p_{1}^{*}\right|=\frac{\left[\left(M_{W}^{2}-\left(m_{f}+m_{F}\right)^{2}\right)\left(M_{W}^{2}-\left(m_{f}-m_{F}\right)^{2}\right)\right]^{1 / 2}}{2 M_{W}} \\
& \left|p_{3}\right|=\frac{\left[\left(\left(2 M_{\chi}\right)^{2}-\left(m_{W}+m_{H}\right)^{2}\right)\left(\left(2 M_{\chi}\right)^{2}-\left(m_{W}-m_{H}\right)^{2}\right)\right]^{1 / 2}}{4 M_{\chi}}
\end{aligned}
$$

One can rewrite this expression, using that the two-to-two and $\mathrm{W}$ decay rate are given by

$$
\begin{aligned}
\sigma v_{H W} & =\frac{1}{8 \pi} \frac{\left|p_{3}\right|}{\left(2 M_{\chi}\right)^{2}} \sum_{\lambda}\left|\mathcal{M}_{2 \rightarrow 2}^{\lambda}\right|^{2} \\
\Gamma_{W \rightarrow f \bar{F}} & =\frac{1}{8 \pi} \frac{\left|p_{1}^{*}\right|}{M_{W}^{2}} \overline{\mid \mathcal{M}}_{1 \rightarrow 2}^{2}
\end{aligned}
$$

where $\mathcal{M}_{2 \rightarrow 2}^{\lambda}$ is the helicity amplitude and ${\overline{\left|\mathcal{M}_{1 \rightarrow 2}\right|}}^{2}$ is the usual summed/averaged decay matrix element. Expressed in terms of the matrix introduced above, ${\overline{\left|\mathcal{M}_{1 \rightarrow 2}\right|}}^{2}=\frac{1}{3} \sum_{\lambda} \mathcal{D}_{\lambda \lambda}$. 
The dependence on the angle cancels in this sum. Then,

$$
\begin{aligned}
\frac{d\left(\sigma v_{H f \bar{F}}\right)^{N W A}}{d \cos \theta} & =\sigma v_{H W} \frac{\Gamma_{W \rightarrow f \bar{F}}}{\Gamma_{W}} \times \frac{{\overline{\mid \mathcal{M}_{\mathrm{res}}}}^{2}}{2\left(\sum_{\lambda}\left|\mathcal{M}_{2 \rightarrow 2}^{\lambda}\right|^{2}\right)\left(\frac{1}{3} \sum_{\lambda} \mathcal{D}_{\lambda \lambda}\right)} \\
& =\sigma v_{H W} B R(W \rightarrow f \bar{F}) \times \mathcal{F}_{\chi \chi \rightarrow H W \rightarrow H f \bar{F}}(\theta)
\end{aligned}
$$

where we have defined the function $\mathcal{F}$ which characterizes the angular dependence. Using also (C.4) for $\mathcal{M}_{\text {res }}$, one can write it as

$$
\mathcal{F}_{\chi \chi \rightarrow H W \rightarrow H f \bar{F}}(\theta)=\frac{\sum_{\lambda, \lambda^{\prime}} \mathcal{M}_{2 \rightarrow 2}^{* \lambda^{\prime}} \mathcal{M}_{2 \rightarrow 2}^{\lambda} \times \mathcal{D}_{\lambda \lambda^{\prime}}(\theta)}{2\left(\sum_{\lambda}\left|\mathcal{M}_{2 \rightarrow 2}^{\lambda}\right|^{2}\right)\left(\frac{1}{3} \sum_{\lambda} \mathcal{D}_{\lambda \lambda}\right)} .
$$

If one would replace the matrix $\mathcal{D}_{\lambda \lambda^{\prime}}$ by the decorrelated approximation (C.8), the last term becomes constant

$$
\left.\mathcal{F}_{\chi \chi \rightarrow H W \rightarrow H f \bar{F}}(\theta)\right|_{\mathcal{D} \rightarrow \mathcal{D}^{\text {decorrelated }}}=\frac{1}{2} .
$$

Integrating over the angle $d \cos \theta$ (which yields a factor 2), one then recovers the familiar relation for the NWA of the total cross section. However, in general the matrix $\mathcal{D}_{\lambda \lambda^{\prime}}$ differs from the decorrelated approximation, and has a non-trivial angular dependence as well as off-diagonal entries.

For Majorana DM annihilation into a scalar and a vector, only the longitudinal polarization contributes to the $s$-wave, i.e. $\mathcal{M}_{2 \rightarrow 2}^{\lambda= \pm 1} \rightarrow 0$ for $v \rightarrow 0$. Using the explicit expression for $\mathcal{D}_{\lambda \lambda^{\prime}}$, this imples that

$$
\mathcal{F}_{\chi \chi \rightarrow H W \rightarrow H f \bar{F}}^{s-\text { wave }}(\theta)=\frac{\mathcal{D}_{00}(\theta)}{2 \frac{1}{3} \sum_{\lambda} \mathcal{D}_{\lambda \lambda}}=\frac{M_{W}^{2}-m_{f}^{2}-m_{F}^{2}-4\left|p_{1}^{*}\right|^{2} \cos ^{2} \theta}{2\left(M_{W}^{2}-m_{f}^{2}-m_{F}^{2}-\frac{4}{3}\left|p_{1}^{*}\right|^{2}\right)} \approx \frac{3 \sin ^{2} \theta}{4},
$$

where the last expression applies for massless fermions. This corresponds to the decay spectrum of a longitudinally polarized $W$ boson. The integral of this expression over $d \cos \theta$ coincides with the decorrelated case. Therefore, the result for the total cross section in the NWA is nevertheless accurate, with error governed by $\Gamma_{W} / M_{W}$, as expected.

Instead of the angle $\theta$ one can use the energy $E_{f}$ of the fermion in the rest frame of the annihilating particles,

$$
E_{f}=\gamma\left(\sqrt{\left|p_{1}^{*}\right|^{2}+m_{f}^{2}}+\left|p_{1}^{*}\right| \beta \cos \theta\right), \quad d E_{f}=\gamma \beta\left|p_{1}^{*}\right| d \cos \theta
$$

where $\beta=\left|p_{3}\right| / \sqrt{\left|p_{3}\right|^{2}+M_{W}^{2}}$ and $\gamma=\left(1-\beta^{2}\right)^{-1 / 2}$. This finally yields the fermion spectrum in the narrow-width limit,

$$
\left(\frac{d \sigma v_{H f \bar{F}}}{d E_{f}}\right)^{N W A}=\frac{\sigma v_{H W} B R(W \rightarrow f \bar{F})}{\gamma \beta\left|p_{1}^{*}\right|} \times\left.\mathcal{F}_{\chi \chi \rightarrow H W \rightarrow H f \bar{F}}(\theta)\right|_{\cos \theta=\frac{E_{f}-\gamma \sqrt{\left|p_{1}^{*}\right|^{2}+m_{f}^{2}}}{\left|p_{1}^{*}\right| \beta \gamma}}
$$

This procedure can be generalized to other three-body final states in a straightforward way. For example, for $\chi \chi \rightarrow W f \bar{F}$, the contribution from the $W$ resonance is

$$
\left.\left(\frac{d \sigma v_{W^{+} f \bar{F}}}{d E_{f}}\right)^{N W A}\right|_{R=W}=\frac{\sigma v_{W W} B R\left(W^{-} \rightarrow f \bar{F}\right)}{\gamma \beta\left|p_{1}^{*}\right|} \times\left.\mathcal{F}_{\chi \chi \rightarrow W W \rightarrow W^{+} f \bar{F}}(\theta)\right|_{\cos \theta=\frac{E_{f}-\gamma \sqrt{\left.||_{1}^{*}\right|^{2}+m_{f}^{2}}}{\left|p_{1}^{*}\right| \beta \gamma}(\mathrm{C} .33)}
$$


where

$$
\mathcal{F}_{\chi \chi \rightarrow W W \rightarrow W+f \bar{F}}(\theta)=\frac{\sum_{\lambda, \lambda^{\prime}, \lambda_{3}} \mathcal{M}_{2 \rightarrow 2}^{* \lambda_{3} \lambda^{\prime}} \mathcal{M}_{2 \rightarrow 2}^{\lambda_{3} \lambda} \times \mathcal{D}_{\lambda \lambda^{\prime}}(\theta)}{2\left(\sum_{\lambda, \lambda_{3}}\left|\mathcal{M}_{2 \rightarrow 2}^{\lambda_{3} \lambda}\right|^{2}\right)\left(\frac{1}{3} \sum_{\lambda} \mathcal{D}_{\lambda \lambda}\right)},
$$

and $\mathcal{M}_{2 \rightarrow 2}^{\lambda_{3} \lambda}=\epsilon_{\mu}^{\lambda_{3}}\left(p_{3}\right) \epsilon_{\mu}^{\lambda}(q) \mathcal{M}_{2 \rightarrow 2}^{\mu \nu}$ is the helicity amlitude for the $\chi \chi \rightarrow W W$ annihilation process. In comparison to before, we have to sum in addition over the polarizations of the $W^{+}$that appears in the three-body final state. The matrix $\mathcal{D}_{\lambda \lambda^{\prime}}(\theta)$ is the same as before.

For $s$-wave annihilation the pair of vector bosons is in a state with $S=L=1, J=0$, and $L_{z}=S_{z}=0$, when choosing the $z$-axis along the momentum of one of the final state particles. The possible spin projections $m_{1}$ and $m_{2}$ of the vector bosons are then determined by the Clebsch-Gordon coefficients for coupling two spin-1 states $\left(S_{1}=S_{2}=1\right)$ to a total spin $S=1$ state with $m \equiv S_{z}=0$,

$$
\left\langle S_{1} S_{2} ; m_{1} m_{2} \mid S_{1} S_{2} ; S m\right\rangle= \begin{cases}\sqrt{1 / 2} & m_{1}=1, m_{2}=-1 \\ 0 & m_{1}=0, m_{2}=0 \\ -\sqrt{1 / 2} & m_{1}=1, m_{2}=-1\end{cases}
$$

Since the spatial momenta of the vectors are opposite, this means they can only be in equal helicity states, and additionally have to be transverse, more precisely

$$
\mathcal{M}_{p}^{\lambda \lambda^{\prime}} \propto\left\langle 11 ; \lambda\left(-\lambda^{\prime}\right) \mid 11 ; 10\right\rangle \propto \operatorname{diag}(1,0,-1)
$$

which implies

$$
\begin{aligned}
\mathcal{F}_{\chi \chi \rightarrow W W \rightarrow W^{+} f \bar{F}}^{s-\text { wave }}(\theta) & =\frac{M_{W}^{2}-m_{f}^{2}-m_{F}^{2}-2\left|p_{1}^{*}\right|^{2} \sin ^{2} \theta}{2\left(M_{W}^{2}-m_{f}^{2}-m_{F}^{2}-\frac{4}{3}\left|p_{1}^{*}\right|^{2}\right)} \\
& \approx \frac{3}{4}\left(1-\frac{1}{2} \sin ^{2} \theta\right) .
\end{aligned}
$$

This corresponds to the decay spectrum of transversely polasized $W$ bosons, and the last line applies to massless fermions. It is straightforward to derive the corresponding matrix $\mathcal{D}_{\lambda \lambda^{\prime}}$ for $Z$ decay and to generalize the procedure to a fermionic (top) resonance. One finds similarly that due to $\mathrm{CP}$ and angular momentum conservation only a definite helicity state can contribute, which then determines the decay spectrum.

Open Access. This article is distributed under the terms of the Creative Commons Attribution License (CC-BY 4.0), which permits any use, distribution and reproduction in any medium, provided the original author(s) and source are credited.

\section{References}

[1] Planck collaboration, P.A.R. Ade et al., Planck 2015 results XIII. Cosmological parameters, Astron. Astrophys. 594 (2016) A13 [arXiv:1502.01589] [INSPIRE].

[2] G. Bertone, Particle dark matter: observations, models and searches, Cambridge University Press, Cambridge U.K., (2010). 
[3] B.W. Lee and S. Weinberg, Cosmological lower bound on heavy neutrino masses, Phys. Rev. Lett. 39 (1977) 165 [INSPIRE].

[4] P. Gondolo and G. Gelmini, Cosmic abundances of stable particles: improved analysis, Nucl. Phys. B 360 (1991) 145 [INSPIRE].

[5] LUX collaboration, D.S. Akerib et al., Improved limits on scattering of weakly interacting massive particles from reanalysis of 2013 LUX data, Phys. Rev. Lett. 116 (2016) 161301 [arXiv: 1512.03506] [INSPIRE].

[6] PandaX-II collaboration, A. Tan et al., Dark matter results from first 98.7 days of data from the PandaX-II experiment, Phys. Rev. Lett. 117 (2016) 121303 [arXiv:1607.07400] [INSPIRE].

[7] ATLAS collaboration, Dark matter interpretations of ATLAS searches for the electroweak production of supersymmetric particles in $\sqrt{s}=8 \mathrm{TeV}$ proton-proton collisions, JHEP 09 (2016) 175 [arXiv: 1608.00872] [INSPIRE].

[8] CMS collaboration, Phenomenological MSSM interpretation of CMS searches in pp collisions at $\sqrt{s}=7$ and $8 \mathrm{TeV}$, JHEP 10 (2016) 129 [arXiv:1606.03577] [INSPIRE].

[9] Fermi-LAT collaboration, M. Ackermann et al., Searching for dark matter annihilation from milky way dwarf spheroidal galaxies with six years of Fermi Large Area Telescope data, Phys. Rev. Lett. 115 (2015) 231301 [arXiv:1503.02641] [InSPIRE].

[10] L. Bergstrom, T. Bringmann, I. Cholis, D. Hooper and C. Weniger, New limits on dark matter annihilation from AMS cosmic ray positron data, Phys. Rev. Lett. 111 (2013) 171101 [arXiv: 1306.3983] [INSPIRE].

[11] L. Bergstrom, Radiative processes in dark matter photino annihilation, Phys. Lett. B 225 (1989) 372 [INSPIRE].

[12] R. Flores, K.A. Olive and S. Rudaz, Radiative processes in LSP annihilation, Phys. Lett. B 232 (1989) 377 [INSPIRE].

[13] T. Bringmann, L. Bergstrom and J. Edsjö, New gamma-ray contributions to supersymmetric dark matter annihilation, JHEP 01 (2008) 049 [arXiv:0710.3169] [INSPIRE].

[14] L. Bergstrom, T. Bringmann and J. Edsjö, New positron spectral features from supersymmetric dark matter - a way to explain the PAMELA data?, Phys. Rev. D 78 (2008) 103520 [arXiv:0808.3725] [INSPIRE].

[15] M. Kachelriess, P.D. Serpico and M.A. Solberg, On the role of electroweak bremsstrahlung for indirect dark matter signatures, Phys. Rev. D 80 (2009) 123533 [arXiv:0911.0001] [INSPIRE].

[16] P. Ciafaloni, D. Comelli, A. Riotto, F. Sala, A. Strumia and A. Urbano, Weak corrections are relevant for dark matter indirect detection, JCAP 03 (2011) 019 [arXiv:1009.0224] [INSPIRE].

[17] M. Cirelli et al., PPPC 4 DM ID: a Poor Particle Physicist Cookbook for Dark Matter Indirect Detection, JCAP 03 (2011) 051 [Erratum ibid. 10 (2012) E01] [arXiv:1012.4515] [INSPIRE].

[18] T. Bringmann and F. Calore, Significant enhancement of neutralino dark matter annihilation from electroweak bremsstrahlung, Phys. Rev. Lett. 112 (2014) 071301 [arXiv:1308.1089] [INSPIRE]. 
[19] H. Goldberg, Constraint on the photino mass from cosmology, Phys. Rev. Lett. 50 (1983) 1419 [Erratum ibid. 103 (2009) 099905] [INSPIRE].

[20] F. Luo and T. You, Enhancement of Majorana dark matter annihilation through Higgs bremsstrahlung, JCAP 12 (2013) 024 [arXiv: 1310.5129] [INSPIRE].

[21] T. Toma, Internal bremsstrahlung signature of real scalar dark matter and consistency with thermal relic density, Phys. Rev. Lett. 111 (2013) 091301 [arXiv:1307.6181] [INSPIRE].

[22] F. Giacchino, L. Lopez-Honorez and M.H.G. Tytgat, Scalar dark matter models with significant internal bremsstrahlung, JCAP 10 (2013) 025 [arXiv:1307.6480] [INSPIRE].

[23] A. Ibarra, T. Toma, M. Totzauer and S. Wild, Sharp gamma-ray spectral features from scalar dark matter annihilations, Phys. Rev. D 90 (2014) 043526 [arXiv:1405.6917] [INSPIRE].

[24] F. Giacchino, L. Lopez-Honorez and M.H.G. Tytgat, Bremsstrahlung and gamma-ray lines in 3 scenarios of dark matter annihilation, JCAP 08 (2014) 046 [arXiv: 1405.6921] [INSPIRE].

[25] F. Giacchino, A. Ibarra, L. Lopez Honorez, M.H.G. Tytgat and S. Wild, Signatures from scalar dark matter with a vector-like quark mediator, JCAP 02 (2016) 002 [arXiv: 1511.04452] [INSPIRE].

[26] G. Bambhaniya, J. Kumar, D. Marfatia, A.C. Nayak and G. Tomar, Vector dark matter annihilation with internal bremsstrahlung, Phys. Lett. B 766 (2017) 177 [arXiv: 1609.05369] [INSPIRE].

[27] N.F. Bell, Y. Cai, J.B. Dent, R.K. Leane and T.J. Weiler, Enhancing dark matter annihilation rates with dark bremsstrahlung, Phys. Rev. D 96 (2017) 023011 [arXiv: 1705.01105] [INSPIRE].

[28] G. Jungman, M. Kamionkowski and K. Griest, Supersymmetric dark matter, Phys. Rept. 267 (1996) 195 [hep-ph/9506380] [INSPIRE].

[29] T. Bringmann, A.J. Galea and P. Walia, Leading QCD corrections for indirect dark matter searches: a fresh look, Phys. Rev. D 93 (2016) 043529 [arXiv: 1510.02473] [InSPIRE].

[30] P. Ciafaloni, M. Cirelli, D. Comelli, A. De Simone, A. Riotto and A. Urbano, On the importance of electroweak corrections for Majorana dark matter indirect detection, JCAP 06 (2011) 018 [arXiv: 1104.2996] [INSPIRE].

[31] N.F. Bell, J.B. Dent, A.J. Galea, T.D. Jacques, L.M. Krauss and T.J. Weiler, $W / Z$ bremsstrahlung as the dominant annihilation channel for dark matter, revisited, Phys. Lett. B 706 (2011) 6 [arXiv:1104.3823] [INSPIRE].

[32] N.F. Bell, J.B. Dent, T.D. Jacques and T.J. Weiler, Dark matter annihilation signatures from electroweak bremsstrahlung, Phys. Rev. D 84 (2011) 103517 [arXiv:1101.3357] [INSPIRE].

[33] M. Garny, A. Ibarra and S. Vogl, Antiproton constraints on dark matter annihilations from internal electroweak bremsstrahlung, JCAP 07 (2011) 028 [arXiv:1105.5367] [INSPIRE].

[34] K. Shudo and T. Nihei, Electroweak bremsstrahlung in binolike dark matter annihilations, Phys. Rev. D 88 (2013) 055019 [arXiv:1306.5901] [INSPIRE].

[35] L.A. Cavasonza, M. Krämer and M. Pellen, Electroweak fragmentation functions for dark matter annihilation, JCAP 02 (2015) 021 [arXiv:1409.8226] [INSPIRE].

[36] M. Garny, A. Ibarra and S. Vogl, Dark matter annihilations into two light fermions and one gauge boson: general analysis and antiproton constraints, JCAP 04 (2012) 033 [arXiv:1112.5155] [INSPIRE]. 
[37] P. Ciafaloni, D. Comelli, A. De Simone, A. Riotto and A. Urbano, Electroweak bremsstrahlung for wino-like dark matter annihilations, JCAP 06 (2012) 016 [arXiv:1202.0692] [INSPIRE].

[38] J. Kumar, J. Liao and D. Marfatia, Dark matter annihilation with s-channel internal Higgsstrahlung, Phys. Lett. B 759 (2016) 277 [arXiv: 1605.00611] [INSPIRE].

[39] P. Gondolo, J. Edsjö, P. Ullio, L. Bergstrom, M. Schelke and E.A. Baltz, DarkSUSY: computing supersymmetric dark matter properties numerically, JCAP 07 (2004) 008 [astro-ph/0406204] [INSPIRE].

[40] T. Bringmann, J. Edsjö, P. Gondolo, P. Ullio and L. Bergstrom, DarkSUSY 6.0: an advanced tool to compute dark matter properties numerically, in preparation, (2017).

[41] J. Kumar and D. Marfatia, Matrix element analyses of dark matter scattering and annihilation, Phys. Rev. D 88 (2013) 014035 [arXiv:1305.1611] [InSPIRE].

[42] C. Becchi, A. Rouet and R. Stora, Renormalization of the Abelian Higgs-Kibble model, Commun. Math. Phys. 42 (1975) 127 [INSPIRE].

[43] C. Becchi, A. Rouet and R. Stora, Renormalization of gauge theories, Annals Phys. 98 (1976) 287 [INSPIRE].

[44] K. Fujikawa, B.W. Lee and A.I. Sanda, Generalized renormalizable gauge formulation of spontaneously broken gauge theories, Phys. Rev. D 6 (1972) 2923 [INSPIRE].

[45] J. Edsjö, Aspects of neutrino detection of neutralino dark matter, Ph.D. thesis, Uppsala U., Uppsala Sweden, (1997) [hep-ph/9704384] [INSPIRE].

[46] X.-L. Chen and M. Kamionkowski, Three body annihilation of neutralinos below two-body thresholds, JHEP 07 (1998) 001 [hep-ph/9805383] [INSPIRE].

[47] C.E. Yaguna, Large contributions to dark matter annihilation from three-body final states, Phys. Rev. D 81 (2010) 075024 [arXiv:1003.2730] [INSPIRE].

[48] G. Bélanger, F. Boudjema, A. Pukhov and A. Semenov, MicrOMEGAs 3 : a program for calculating dark matter observables, Comput. Phys. Commun. 185 (2014) 960 [arXiv: 1305.0237] [INSPIRE].

[49] Particle Data Group collaboration, K.A. Olive et al., Review of particle physics, Chin. Phys. C 38 (2014) 090001 [inSPIRE].

[50] C.F. Uhlemann and N. Kauer, Narrow-width approximation accuracy, Nucl. Phys. B 814 (2009) 195 [arXiv:0807.4112] [INSPIRE].

[51] J. Chakrabortty, A. Kundu and T. Srivastava, Novel method to deal with off-shell particles in cascade decays, Phys. Rev. D 93 (2016) 053005 [arXiv:1601.02375] [INSPIRE].

[52] J. Chakrabortty, A. Kundu, R. Maji and T. Srivastava, Cut and compute: quick cascades with multiple amplitudes, arXiv:1703.07174 [INSPIRE].

[53] F. Bloch and A. Nordsieck, Note on the radiation field of the electron, Phys. Rev. 52 (1937) 54 [INSPIRE].

[54] T. Kinoshita, Mass singularities of Feynman amplitudes, J. Math. Phys. 3 (1962) 650 [INSPIRE].

[55] M. Bauer, T. Cohen, R.J. Hill and M.P. Solon, Soft collinear effective theory for heavy WIMP annihilation, JHEP 01 (2015) 099 [arXiv:1409.7392] [INSPIRE]. 
[56] G. Ovanesyan, T.R. Slatyer and I.W. Stewart, Heavy dark matter annihilation from effective field theory, Phys. Rev. Lett. 114 (2015) 211302 [arXiv:1409.8294] [INSPIRE].

[57] M. Baumgart, I.Z. Rothstein and V. Vaidya, Calculating the annihilation rate of weakly interacting massive particles, Phys. Rev. Lett. 114 (2015) 211301 [arXiv:1409.4415] [INSPIRE].

[58] J. Hisano, S. Matsumoto and M.M. Nojiri, Explosive dark matter annihilation, Phys. Rev. Lett. 92 (2004) 031303 [hep-ph/0307216] [INSPIRE].

[59] M. Beneke, C. Hellmann and P. Ruiz-Femenia, Non-relativistic pair annihilation of nearly mass degenerate neutralinos and charginos III. Computation of the Sommerfeld enhancements, JHEP 05 (2015) 115 [arXiv:1411.6924] [INSPIRE].

[60] M. Beneke, A. Bharucha, A. Hryczuk, S. Recksiegel and P. Ruiz-Femenia, The last refuge of mixed wino-Higgsino dark matter, JHEP 01 (2017) 002 [arXiv: 1611.00804] [INSPIRE].

[61] T. Sjöstrand, S. Mrenna and P.Z. Skands, A brief introduction to PYTHIA 8.1, Comput. Phys. Commun. 178 (2008) 852 [arXiv:0710.3820] [INSPIRE].

[62] M. Asano, T. Bringmann, G. Sigl and M. Vollmann, $130 \mathrm{GeV}$ gamma-ray line and generic dark matter model building constraints from continuum gamma rays, radio and antiproton data, Phys. Rev. D 87 (2013) 103509 [arXiv:1211.6739] [InSPIRE].

[63] F. Feroz, M.P. Hobson and M. Bridges, MultiNest: an efficient and robust Bayesian inference tool for cosmology and particle physics, Mon. Not. Roy. Astron. Soc. 398 (2009) 1601 [arXiv:0809.3437] [INSPIRE].

[64] J. Edsjö and P. Gondolo, Neutralino relic density including coannihilations, Phys. Rev. D 56 (1997) 1879 [hep-ph/9704361] [INSPIRE].

[65] J. Edsjö, M. Schelke, P. Ullio and P. Gondolo, Accurate relic densities with neutralino, chargino and sfermion coannihilations in mSUGRA, JCAP 04 (2003) 001 [hep-ph/0301106] [INSPIRE].

[66] ATLAS and CMS collaborations, Combined measurement of the Higgs boson mass in pp collisions at $\sqrt{s}=7$ and $8 \mathrm{TeV}$ with the ATLAS and CMS experiments, Phys. Rev. Lett. 114 (2015) 191803 [arXiv: 1503.07589] [INSPIRE].

[67] CMS collaboration, Searches for supersymmetry using the $M_{\mathrm{T} 2}$ variable in hadronic events produced in pp collisions at 8 TeV, JHEP 05 (2015) 078 [arXiv: 1502.04358] [INSPIRE].

[68] CMS collaboration, Exclusion limits on gluino and top-squark pair production in natural SUSY scenarios with inclusive razor and exclusive single-lepton searches at $8 \mathrm{TeV}$, CMS-PAS-SUS-14-011, CERN, Geneva Switzerland, (2014).

[69] CMS collaboration, Search for top squarks decaying to a charm quark and a neutralino in events with a jet and missing transverse momentum, CMS-PAS-SUS-13-009, CERN, Geneva Switzerland, (2013).

[70] CMS collaboration, Search for top-squark pair production in the single-lepton final state in pp collisions at $\sqrt{s}=8 \mathrm{TeV}$, Eur. Phys. J. C 73 (2013) 2677 [arXiv:1308.1586] [INSPIRE].

[71] CMS collaboration, Search for top squarks in multijet events with large missing momentum in proton-proton collisions at $8 \mathrm{TeV}$, CMS-PAS-SUS-13-015, CERN, Geneva Switzerland, (2013). 
[72] CMS collaboration, Search for top-squark pairs decaying into Higgs or $Z$ bosons in pp collisions at $\sqrt{s}=8 \mathrm{TeV}$, Phys. Lett. B 736 (2014) 371 [arXiv:1405.3886] [InSPIRE].

[73] CMS collaboration, Search for supersymmetry in pp collisions at $\sqrt{s}=8$ TeV in events with three leptons and at least one b-tagged jet, CMS-PAS-SUS-13-008, CERN, Geneva Switzerland, (2013).

[74] ATLAS collaboration, Search for pair-produced third-generation squarks decaying via charm quarks or in compressed supersymmetric scenarios in pp collisions at $\sqrt{s}=8$ TeV with the ATLAS detector, Phys. Rev. D 90 (2014) 052008 [arXiv:1407.0608] [INSPIRE].

[75] ATLAS collaboration, Search for direct third-generation squark pair production in final states with missing transverse momentum and two b-jets in $\sqrt{s}=8$ TeV pp collisions with the ATLAS detector, JHEP 10 (2013) 189 [arXiv:1308.2631] [INSPIRE].

[76] ATLAS collaboration, Search for direct production of charginos, neutralinos and sleptons in final states with two leptons and missing transverse momentum in pp collisions at $\sqrt{s}=8 \mathrm{TeV}$ with the ATLAS detector, JHEP 05 (2014) 071 [arXiv:1403.5294] [INSPIRE].

[77] ATLAS collaboration, Search for the direct production of charginos, neutralinos and staus in final states with at least two hadronically decaying taus and missing transverse momentum in pp collisions at $\sqrt{s}=8 \mathrm{TeV}$ with the ATLAS detector, JHEP 10 (2014) 096 [arXiv: 1407.0350] [INSPIRE].

[78] LUX collaboration, D.S. Akerib et al., First results from the LUX dark matter experiment at the Sanford Underground Research Facility, Phys. Rev. Lett. 112 (2014) 091303 [arXiv: 1310.8214] [INSPIRE].

[79] GAMBIT collaboration, P. Athron et al., A global fit of the MSSM with GAMBIT, arXiv: 1705.07917 [INSPIRE].

[80] H. Liu, T.R. Slatyer and J. Zavala, Contributions to cosmic reionization from dark matter annihilation and decay, Phys. Rev. D 94 (2016) 063507 [arXiv:1604.02457] [InSPIRE].

[81] AMS collaboration, L. Accardo et al., High statistics measurement of the positron fraction in primary cosmic rays of 0.5-500 GeV with the Alpha Magnetic Spectrometer on the International Space Station, Phys. Rev. Lett. 113 (2014) 121101 [INSPIRE].

[82] K. Fukushima, Y. Gao, J. Kumar and D. Marfatia, Bremsstrahlung signatures of dark matter annihilation in the sun, Phys. Rev. D 86 (2012) 076014 [arXiv:1208.1010] [INSPIRE].

[83] A. Ibarra, M. Totzauer and S. Wild, High-energy neutrino signals from the sun in dark matter scenarios with internal bremsstrahlung, JCAP 12 (2013) 043 [arXiv:1311.1418] [INSPIRE].

[84] A. Ibarra, M. Totzauer and S. Wild, Higher order dark matter annihilations in the sun and implications for IceCube, JCAP 04 (2014) 012 [arXiv: 1402.4375] [INSPIRE].

[85] N.F. Bell, A.J. Brennan and T.D. Jacques, Neutrino signals from electroweak bremsstrahlung in solar WIMP annihilation, JCAP 10 (2012) 045 [arXiv:1206.2977] [INSPIRE].

[86] A. De Simone, A. Monin, A. Thamm and A. Urbano, On the effective operators for dark matter annihilations, JCAP 02 (2013) 039 [arXiv:1301.1486] [INSPIRE].

[87] F. Calore, Unveiling dark matter through gamma rays: spectral features, spatial signatures and astrophysical backgrounds, Ph.D. thesis, Hamburg U., Hamburg Germany, (2013). 
[88] T. Hahn, Generating Feynman diagrams and amplitudes with FeynArts 3, Comput. Phys. Commun. 140 (2001) 418 [hep-ph/0012260] [INSPIRE].

[89] J. Edsjö and P. Gondolo, Neutralino relic density including coannihilations, Phys. Rev. D 56 (1997) 1879 [hep-ph/9704361] [INSPIRE].

[90] T. Hahn, Form Calc 6, PoS (ACAT08) 121 [arXiv:0901.1528] [InSPIRE].

[91] M. Jacob and G.C. Wick, On the general theory of collisions for particles with spin, Annals Phys. 7 (1959) 404 [Annals Phys. 281 (2000) 774] [INSPIRE].

[92] P. Ciafaloni, M. Cirelli, D. Comelli, A. De Simone, A. Riotto and A. Urbano, Initial state radiation in Majorana dark matter annihilations, JCAP 10 (2011) 034 [arXiv:1107.4453] [INSPIRE].

[93] A. Belyaev, N.D. Christensen and A. Pukhov, CalcHEP 3.4 for collider physics within and beyond the Standard Model, Comput. Phys. Commun. 184 (2013) 1729 [arXiv:1207.6082] [INSPIRE].

[94] B.C. Allanach, SOFTSUSY: a program for calculating supersymmetric spectra, Comput. Phys. Commun. 143 (2002) 305 [hep-ph/0104145] [INSPIRE].

[95] P. Gondolo, J. Edsjö, P. Ullio, L. Bergstrom, M. Schelke and E.A. Baltz, DarkSUSY: computing supersymmetric dark matter properties numerically, JCAP 07 (2004) 008 [astro-ph/0406204] [INSPIRE]. 